\title{
TENSOR-SPARSITY OF SOLUTIONS TO HIGH-DIMENSIONAL ELLIPTIC PARTIAL DIFFERENTIAL EQUATIONS
}

\author{
WOLFGANG DAHMEN, RONALD DEVORE, LARS GRASEDYCK, AND ENDRE SÜLI
}

\begin{abstract}
A recurring theme in attempts to break the curse of dimensionality in the numerical approximations of solutions to high-dimensional partial differential equations (PDEs) is to employ some form of sparse tensor approximation. Unfortunately, there are only a few results that quantify the possible advantages of such an approach. This paper introduces a class $\Sigma_{n}$ of functions, which can be written as a sum of rank-one tensors using a total of at most $n$ parameters and then uses this notion of sparsity to prove a regularity theorem for certain high-dimensional elliptic PDEs. It is shown, among other results, that whenever the right-hand side $f$ of the elliptic PDE can be approximated with a certain rate $\mathcal{O}\left(n^{-r}\right)$ in the norm of $\mathrm{H}^{-1}$ by elements of $\Sigma_{n}$, then the solution $u$ can be approximated in $\mathrm{H}^{1}$ from $\Sigma_{n}$ to accuracy $\mathcal{O}\left(n^{-r^{\prime}}\right)$ for any $r^{\prime} \in(0, r)$. Since these results require knowledge of the eigenbasis of the elliptic operator considered, we propose a second "basis-free" model of tensor sparsity and prove a regularity theorem for this second sparsity model as well. We then proceed to address the important question of the extent such regularity theorems translate into results on computational complexity. It is shown how this second model can be used to derive computational algorithms with performance that breaks the curse of dimensionality on certain model high-dimensional elliptic PDEs with tensor-sparse data.
\end{abstract}

AMS Subject Classification: 35J25, 41A25, 41A63, 41A46, 65D99

Key Words: High-dimensional elliptic PDEs, tensor sparsity models, regularity theorems, exponential sums of operators, Dunford integrals, complexity bounds

\section{INTRODUCTION}

Many important problems that arise in applications involve a large number of spatial variables. These high-dimensional problems pose a serious computational challenge because of the so-called curse of dimensionality; roughly speaking, this means that when using classical methods of approximation or numerical methods based on classical approximations, the computational work required to approximate or to recover a function of $d$ variables with a desired target accuracy typically scales exponentially in $d$. This has led to so-called intractability results, which say that even under the assumption that the target function has very high order of classical regularity (in terms of various notions of derivatives), the exponential effect of the spatial dimension $d$ prevails, see [30]. Subsequent attempts to overcome the curse of dimensionality have been mainly based on exploring the effect of very strong regularity assumptions, or constraining the dependence of the functions on some of the variables, [29]. It is not clear though, for which problems of practical interest such strong assumptions are actually satisfied.

The tacit assumption behind these negative tractability results is that classical notions of smoothness are used to characterize the regularity of the solution and classical methods of approximation are used in the development of the numerical algorithms. In low spatial dimensions, smoothness is typically exploited by using classical approximation methods, such as splines or finite elements, based on localization. This is often enhanced by adaptation concepts, which exploit

Date: September 7, 2018.

This work has been supported in part by the DFG Special Priority Program SPP-1324, by the DFG SFBTransregio 40, by the DFG Research Group 1779, the Excellence Initiative of the German Federal and State Governments, (RWTH Aachen Distinguished Professorship, Graduate School AICES), and NSF grant DMS 1222390. The second author's research was supported by the Office of Naval Research Contracts ONR N00014-09-1-0107, ONR N00014-11-1-0712, ONR N00014-12-1-0561; and by the NSF Grants DMS 0915231, DMS 1222715. This research was initiated when he was an AICES Visiting Professor at RWTH Aachen. 
weaker smoothness measures in the sense of Besov spaces and thus provide somewhat better convergence rates. However, the larger the spatial dimension the smaller the difference between the various smoothness notions becomes. Adaptivity based on localization is therefore not a decisive remedy, and alternative strategies are called for.

It has recently been recognized that the situation is not as bleak as has been described above. Indeed, solutions to real-world high-dimensional problems are thought to have a structure different from high-dimensional regularity, that renders them more amenable to numerical approximation. The challenge is to explicitly define these new structures, for a given class of problems, and then to build numerical methods that exploit them. This has led to various notions, such as sparsity, variable reduction, and reduced modelling. In contrast with the low-dimensional regime, essentially governed by smoothness, there is of course no universal recipe for discovering the correct notion of sparsity: the correct structural sparsity will depend on the problem at hand.

In the context of numerical approximation of PDEs, there are roughly two groups of highdimensional problems. The first group involves parameter-dependent families of partial differential equations, where the number of "differential" variables is still small but the data and the coefficients in the PDE depend on possibly many additional parameters, which often are to be optimized in a design or optimization context. Hence the solution becomes a function of the spatial variables and of the additional parameters. In particular, such parameter-dependent PDEs are obtained when coefficients in the PDE are random fields. Expanding such a random field may even lead to an infinite number of deterministic parameters, see e.g. [8, 9]. Reduced-order modeling concepts such as POD (proper orthogonal decomposition) or the reduced-basis method aim at constructing solution-dependent dictionaries comprised of judiciously chosen "snapshots" from the solution manifold. This can actually be viewed as a separation ansatz between the spatial variables and the parameters.

The second group of problems, and those of interest to us here, concern partial differential equations posed in a phase space of large spatial dimension (e.g. Schrödinger, Ornstein-Uhlenbeck, Smoluchowski, and Fokker-Planck equations). As the underlying domain $D$ is typically a Cartesian product $D=\times_{j=1}^{d} D_{j}$ of low-dimensional domains, $D_{j}, j=1, \ldots, d$, it is natural to seek (approximate) representations in terms of separable functions - viz. low-rank tensors. Kolmogorov equations and related PDEs in (countably) infinitely many space dimensions, which arise as evolution equations for the probability density function for stochastic PDEs, require a different functionalanalytic setting from the one considered here and are therefore not treated in the present paper; for further details in this direction the reader is referred to [32].

The main purpose of the present paper is to propose specific notions of sparsity, based on tensor decompositions, and then to show that the solutions of certain high-dimensional diffusion equations inherit this type of sparsity from given data. This is then shown to lead indeed to tractability of solving such high-dimensional PDEs.

To motivate the results that follow we sketch a simple example, albeit not in a PDE context yet, which indicates how tensor structure can mitigate the curse of dimensionality. Suppose that $f \in C^{s}\left([0,1]^{d}\right)$ for some $s \in \mathbb{N}$. Approximating $\int_{[0,1]^{d}} f(x) \mathrm{d} x$ by a standard tensor-product quadrature method $I_{s, n}^{d}(f)$ of order $s$ on a Cartesian grid with meshsize $n^{-1}$ in each of the $d$ coordinate directions (e.g. with an sth order accurate composite Newton-Cotes or Gauß quadrature possessing nonnegative weights) yields accuracy in the sense that

$$
\left|\int_{[0,1]^{d}} f(x) \mathrm{d} x-I_{s, n}^{d}(f)\right| \leq C d n^{-s}\|f\|_{C^{s}\left([0,1]^{d}\right)},
$$

at the expense of the order of $N=(s n)^{d}$ operations. Here $C$ is a fixed constant depending on the univariate quadrature rule. If, in addition, one knows that $f$ is a product of given univariate functions: $f(x)=f_{1}\left(x_{1}\right) \cdots f_{d}\left(x_{d}\right)$, i.e., $f$ is a rank-one tensor or separable function, then $\int_{[0,1]^{d}} f(x) \mathrm{d} x=\prod_{j=1}^{d}\left(\int_{0}^{1} f_{j}\left(x_{j}\right) \mathrm{d} x_{j}\right)$, and one obtains

$$
\left|\int_{[0,1]^{d}} f(x) \mathrm{d} x-\prod_{j=1}^{d} I_{s, n}^{1}\left(f_{j}\right)\right| \leq C d n^{-s}\|f\|_{C^{s}\left([0,1]^{d}\right)}
$$


at the expense of the order of $d s n$ operations only, where again $C$ is a fixed constant depending on the univariate quadrature rule.

Thus, in the first case, accuracy $\varepsilon$ costs

$$
N_{1}(\varepsilon)=\mathcal{O}\left(s^{d} d^{d / s} \varepsilon^{-d / s}\right)
$$

operations so that the curse is apparent in the factor $d$ appearing in the power of $\varepsilon$. One could do much better by employing Smolyak quadrature rules, which, however, requires the assumption of significantly higher regularity of the integrand, [29], in order to obtain specific rates. In the second case, accuracy $\varepsilon$ is achieved at the expense of the order of $d^{1+1 / s} s \varepsilon^{-1 / s}$ operations. Thus, one has the full one-dimensional gain of smoothness and the spatial dimension enters only linearly as opposed to exponentially as in the first case. Of course, assuming that $f$ is a rank-one tensor is rather restrictive. If however one knew that it takes only $r(\varepsilon)$ rank-one summands to approximate $f$ to within accuracy $\varepsilon$, the computational cost would still be of the order of

$$
N_{2}(\varepsilon)=r(\varepsilon) d^{1+1 / s} s \varepsilon^{-1 / s}
$$

operations. This is preferable to $N_{1}(\varepsilon)=\mathcal{O}\left(s^{d} \varepsilon^{-d / s}\right)$, even when $r(\varepsilon)$ grows like $\varepsilon^{-\alpha}$ for some fixed positive $\alpha$.

The main purpose of this paper is to show that the type of saving exhibited in the above example of numerical integration is present in the numerical approximation of certain high-dimensional elliptic equations described in $\S 2$. To expect such savings, the elliptic operator under consideration should exhibit a "tensor-friendly" structure. A differential operator that is a tensor-product of low-dimensional elliptic operators would trivially be "tensor-friendly", but it would lead us into classes of hypo-elliptic problems. Here, instead, we consider elliptic operators, which are sums of tensor-product operators. The high-dimensional Laplacian is a prototypical example. We work in a somewhat more general setting than that of a simple Laplace operator for the following reasons. The original motivation for this work was a class of Fokker-Planck equations, whose numerical treatment, after suitable operator splitting steps, reduces to solving a high-dimensional symmetric elliptic problem over a Cartesian product domain, where the energy space of the elliptic operator is a weighted Sobolev space with a strongly degenerate weight. The setting we shall describe in $\S 2$ covers such situations as well, the aim of considering a general class of elliptic problems being to extract and highlight the relevant structural assumptions.

In $\S 3$, we turn to proposing notions of tensor-sparsity that we will prove are relevant for approximating the solutions to operator equations of the type discussed in the above paragraph and formally introduced in $\S 2$. To briefly describe in this introduction the form such sparsity takes, consider a function space $X=X_{1}\left(D_{1}\right) \otimes \cdots \otimes X_{d}\left(D_{d}\right)$ over a Cartesian product domain $D=\times_{j=1}^{d} D_{j}$. Suppose, only for the sake of simplifying the present discussion, that the component domains $D_{j}, j=1, \ldots, d$, are intervals. A standard way of approximating the elements of $X$ is to start with $d$ (a priori chosen) univariate bases $\Psi_{j}=\left\{\psi_{\nu}^{j}: \nu \in \Lambda_{j}\right\}$ for the spaces $X_{j}, j=1, \ldots, d$, where $\Psi_{1} \otimes \cdots \otimes \Psi_{d}$ is dense in $X$. Examples of such bases could be trigonometric functions, polynomials or wavelets. Hence, the product basis $\Psi=\Psi_{1} \otimes \cdots \otimes \Psi_{d}$ allows us to expand $v \in X$ as

$$
v=\sum_{\nu \in \times_{j=1}^{d} \Lambda_{j}} v_{\nu_{1} \ldots \nu_{d}} \psi_{\nu_{1}}^{1} \otimes \cdots \otimes \psi_{\nu_{d}}^{d} .
$$

We use, here and throughout this paper, the standard multi-index notation

$$
\nu=\left(\nu_{1}, \ldots, \nu_{d}\right), \quad \text { and } \quad \nu \leq \mu \text { means that } \nu_{j} \leq \mu_{j} \text { for } j=1, \ldots, d .
$$

Once we have decided to use $\Psi$ as an expansion system, the standard approach to obtaining a possibly sparse approximation is to retain as few terms of the expansion (1.3) as possible, while still meeting a given accuracy tolerance. This is a nonlinear selection process known as $N$-term approximation, see [11] for a general treatment of $N$-term approximation and [12] for a proposed implementation and analysis in the context of high-dimensional elliptic PDEs. However, using such universal bases $\Psi$, which are independent of the specific $v$, best $N$-term approximation procedures significantly mitigate but do not quite avoid the curse of dimensionality. In fact, while in contrast to conventional isotropic multiresolution approximations, under much stronger (mixed) 
smoothness assumptions, the factor $\varepsilon^{-d / s}$ in (1.1) can be replaced by $\varepsilon^{-1 / s}$, the constants in the corresponding error bounds still exhibit an exponential growth in $d$.

Thus, it is not clear how useful $N$-term approximation with respect to a fixed background tensor product basis will be for treating truly high-dimensional problems. This is to be contrasted by allowing $v$ to be expanded in terms of separable functions where the factors are now allowed to depend on $v$

$$
v=\sum_{k=1}^{\infty} v_{k, 1} \otimes \cdots \otimes v_{k, d}, \quad v_{k, j}=v_{k, j}(v) .
$$

Of particular interest is then the case where, in spite of a possibly moderate smoothness of $v$, the terms in this expansion decay so rapidly that only a few of them suffice in order to meet the target accuracy. Thus we are asking for an alternative structural property of a function of many variables that leads to computational tractability despite the lack of high regularity. This will lead us below to proposing new notions of tensor sparsity.

Of course, to that end, aside from the question for which $v$ such an expansion converges rapidly and how to identify the summands, the ultimate computational cost depends also on how well, i.e., at what cost, can the factors $v_{k, j}$ be approximated, e.g. in terms of the universal univariate bases $\Psi_{j}, j=1, \ldots, d$. Thus, two approximation processes have to be intertwined, which, in the present setting, takes the following form:

$$
v \approx v_{N}:=\sum_{k=1}^{r}\left(\sum_{\nu \in \Gamma_{k, 1}} c_{k, \nu}^{1} \psi_{\nu}^{1}\right) \otimes \cdots \otimes\left(\sum_{\nu \in \Gamma_{k, d}} c_{k, \nu}^{d} \psi_{\nu}^{d}\right)
$$

and ideally, one would like to find $r, \Gamma_{k, j}, c_{k, \nu}^{j}, \nu \in \Gamma_{k, j}, k=1, \ldots, r, j=1, \ldots, d$, subject to

$$
\sum_{k=1}^{r} \sum_{j=1}^{d} \#\left(\Gamma_{k, j}\right) \leq N
$$

so that $\left\|v-v_{N}\right\|_{X}$ is (near-)minimized; see [1, 2], where algorithms are proposed that nearly minimize this error. This is obviously a much more demanding (and much more nonlinear) optimization task than activating the best coefficients in (1.3). In fact, it is not clear that a best approximation in this sense exists. However, if $v$ admits such an expansion, where $r=r(\varepsilon)$ increases slowly with decreasing $\varepsilon$ while the $\#\left(\Gamma_{k, j}\right), k=1, \ldots, r, j=1, \ldots, d$, scale like typical low-dimensional processes for moderate regularity, it is clear that the number of coefficients needed in (1.6) would exhibit essentially the same dependence on $\varepsilon$ as $N_{2}(\varepsilon)$ in the integration example discussed above, and hence would be much smaller than the number of coefficients needed in (1.3), for the same accuracy.

Another way to look at this comparison is to note that when expanding the products of sums in (1.6) one obtains a representation of the form (1.3). However, the coefficients in the tensor array $\left(v_{\nu}\right)$, are strongly dependent, as is exhibited by the fact that they can be written as a sum of a few ( $r$ in number) rank-one tensors. In this sense the tensor $\left(v_{\nu}\right)$ is information sparse, i.e., loosely speaking, its entries $v_{\nu}$ depend "on much fewer parameters".

We are now ready to formulate more precisely the central question in this paper: suppose we are given an elliptic problem over a high-dimensional product domain and suppose that the data (the right-hand side function in the partial differential equation) is tensor-sparse in the sense that it can be approximated by terms of the form (1.6) at a certain rate; then, is the solution $u$ also tensor-sparse, and, if so, at which rate? In other words: in which circumstances does a highly nonlinear process offer significantly higher sparsity than (1.3), and does this break the curse of dimensionality?

In $\S 3$, we shall formalize the above ideas and define sparse tensor structures and their spaces $\Sigma_{n}$, whose elements depend on at most $n$ parameters. Sparse spaces of this type can, in principle, be defined relative to any universal background tensor basis. The most convenient one for us in what follows for the purpose of highlighting the essential mechanisms is however a certain eigenbasis for the elliptic operator under consideration. In particular, this allows us to employ exponential sum approximations to reciprocals of eigenvalues, which turns out to be critical for estimating the effect 
of the inversion of an elliptic operator on the growth of ranks in the approximation of solutions. We then formulate approximation classes for this form of approximation. An important issue for computational considerations are the spaces $\Sigma_{n}(\mathcal{R})$, introduced in that section, that impose restrictions on the positions of the tensor basis that are allowed to participate. In $\S 4$ we present our first main contributions, which are regularity results for elliptic PDEs stated in terms of the tensor-sparsity of $\S 3$.

The above approach is primarily of theoretical interest, as a regularity result. From a numerical perspective its practical relevance is limited by the fact that only in rare cases is an eigenbasis available. Therefore, we propose in $\S 5$ an alternative model for tensor-sparsity, which does not make use of any background basis. However, now sparsity is expressed by approximability in terms of short sums of rank-one functions, which are no longer described by a finite number of parameters but are constrained by a certain excess regularity, which, in turn, will render them computable. Hitherto, regularity has been described by the number of such constrained terms needed to approximate the solution to within a given target accuracy. Thanks to the excess regularity, these constrained rank-one terms can again be approximated to within the same target accuracy by a finite number of parameters. We then proceed to quantify this last principal fact. Again exponential sums serve as a key vehicle. This time however, instead of using them for the construction of separable approximations to reciprocals of eigenvalues, they are used to approximate inverses of operators. In contrast with previous applications of exponential sums in the literature $[18,25]$ for the approximation of inverses of discrete (finite-dimensional) linear operators, it is now crucial to take into account the mapping properties of the operators concerned in terms of Sobolev scales.

In $\S 6$ and $\S 7$ we turn to the question of constructing concrete numerical algorithms which exhibit these gains in numerical efficiency. In certain settings, discussed in that section, we give concrete bounds on the representation and numerical complexity of further approximating the regularity-controlled rank-one sums by analogous finitely parametrized expressions. By representation-complexity we mean the total number of parameters needed to eventually represent a tensor expansion to within the target accuracy. Our main tool, in that section, is the Dunford integral representation of the exponential of a sectorial operator. Again, in contrast with previous such uses (e.g. [16, 17]), it is crucial to apply these representations on the continuous (infinitedimensional) level [10]. It is shown that the spatial dimension $d$ enters the (total) complexity only in a moderate superlinear fashion, and thereby the curse of dimensionality is avoided. The results presented here differ in essential ways from those in [35] since the required information on the data is highly nonlinear and the differential operators cannot be diagonalized on the data classes under consideration. Thus, the main point is the complexity of a structure preserving approximate inversion of the operator.

We close the paper with some comments on open problems in $\S 9$.

\section{The General Setting}

2.1. A class of elliptic problems. In this section, we formulate a class of high-dimensional problems for which we will prove that their solutions can be effectively captured by sparse tensor approximations for suitable right-hand sides $f$.

Let $D=\times_{j=1}^{d} D_{j}$ be a Cartesian product domain in $\mathbb{R}^{d p}$ where the $D_{j} \subset \mathbb{R}^{p}$ are low-dimensional domain factors. Typically, $p$ takes the value 1,2 , or 3 . So, the high-dimensionality occurs because $d$ is large. For each $j=1, \ldots, d$, we denote by $H_{j}$ a separable Hilbert space, with norm $\|\cdot\|_{H_{j}}$, comprised of functions defined on $D_{j}$. We assume that each $H_{j}$ is continuously and densely embedded in $L_{2}\left(D_{j}\right)=L_{2}\left(D_{j}, \mu_{j}\right)$ with $\mu_{j}$ a positive Borel measure on the factor domain $D_{j}$ that is absolutely continuous with respect to the Lebesgue measure. Thus, the measures $\mu_{j}$, $j=1, \ldots, d$, are not necessarily the Lebesque measure but could involve weights that are positive a.e. on $D_{j}, j=1, \ldots, d$. The dual pairing $\langle\cdot, \cdot\rangle$ is always understood to be induced by the inner product for $L_{2}\left(D_{j}\right)$. It will be clear from the context whether $\langle\cdot, \cdot\rangle$ denotes a dual pairing or the $L_{2}$ inner product. Thus we have the Gel'fand triple (rigged Hilbert space)

$$
H_{j} \subset L_{2}\left(D_{j}\right) \subset\left(H_{j}\right)^{\prime}, \quad j=1, \ldots, d,
$$


with continuous and dense embeddings, where $\left(H_{j}\right)^{\prime}$ denotes the normed dual of $H_{j}$. We can think of the $H_{j}$ to stand for a (possibly weighted) Sobolev space, and possibly incorporating boundary conditions.

We assume that we are given "low-dimensional" symmetric $H_{j}$-elliptic operators $\mathfrak{B}_{j}: H_{j} \rightarrow$ $\left(H_{j}\right)^{\prime}$, i.e., the bilinear forms $\mathfrak{b}_{j}(v, w):=\left\langle\mathfrak{B}_{j} v, w\right\rangle$ satisfy, for some fixed positive constants $c, \alpha$, the following inequalities:

$$
\left|\mathfrak{b}_{j}(v, w)\right| \leq c\|v\|_{H_{j}}\|w\|_{H_{j}}, \quad \mathfrak{b}_{j}(v, v) \geq \alpha\|v\|_{H_{j}}^{2}, \quad v, w \in H_{j}, j=1, \ldots, d,
$$

and $\mathfrak{b}_{j}(v, w)=\mathfrak{b}_{j}(w, v)$ for all $v, w \in H_{j}$ and all $j=1, \ldots, d$.

We next introduce a Hilbert space $\mathrm{H}$ over $D$ for which we will formulate the high-dimensional elliptic variational problems of interest to us. For each $j=1,2, \ldots, d$, we consider the separable Hilbert space

$$
\mathrm{H}_{j}:=\mathrm{H}_{j}(D):=L_{2}\left(D_{1}\right) \otimes \cdots \otimes L_{2}\left(D_{j-1}\right) \otimes H_{j} \otimes L_{2}\left(D_{j+1}\right) \otimes \cdots \otimes L_{2}\left(D_{d}\right),
$$

(not to be confused with $H_{j}$ ), with its natural norm $\|\cdot\|_{\mathrm{H}_{j}}$ and inner product. From these component spaces, we define

$$
\mathrm{H}:=\bigcap_{j=1}^{d} \mathrm{H}_{j},
$$

which we equip with the norm $\|\cdot\|_{\mathrm{H}}$, defined by

$$
\|v\|_{\mathrm{H}}^{2}:=\sum_{j=1}^{d}\|v\|_{\mathrm{H}_{j}}^{2} .
$$

In the following we will introduce and use an equivalent norm for $\mathrm{H}$ based on eigenexpansions. Again, this gives rise to a Gel'fand triple

$$
\mathrm{H} \subset \mathrm{L}_{2}(D) \subset \mathrm{H}^{\prime},
$$

with dense continuous embeddings. For example, if $\mu_{j}$ is the Lebesgue measure on $D_{j}$ and $H_{j}$ is $H^{1}\left(D_{j}\right), j=1, \ldots, d$, then $\mathrm{H}$ is identical to the standard Sobolev space $H^{1}(D)$, and the above norm is equivalent to the usual $H^{1}(D)$ norm.

The bilinear form on the space $\mathrm{H} \times \mathrm{H}$, under consideration, is given by

$$
\mathfrak{b}(v, w):=\sum_{j=1}^{d}\left\langle\left(\mathcal{I}_{1} \otimes \cdots \otimes \mathcal{I}_{j-1} \otimes \mathfrak{B}_{j} \otimes \mathcal{I}_{j+1} \otimes \cdots \otimes \mathcal{I}_{d}\right) v, w\right\rangle, \quad v, w \in \mathrm{H},
$$

where $\mathcal{I}_{j}$ is the identity operator on $L_{2}\left(D_{j}\right), j=1, \ldots, d$. This form is H-elliptic, i.e., there exist constants $0<c_{a}, c_{a}^{\prime}<\infty$ such that

$$
|\mathfrak{b}(v, w)| \leq c_{a}\|v\|_{\mathrm{H}}\|w\|_{\mathrm{H}}, \quad \mathfrak{b}(v, v) \geq c_{a}^{\prime}\|v\|_{\mathrm{H}}^{2} \quad \forall v, w \in \mathrm{H} .
$$

Furthermore, $\mathfrak{b}$ is symmetric; i.e., $\mathfrak{b}(v, w)=\mathfrak{b}(w, v)$ for all $w, v \in \mathrm{H}$. Thus, the symmetric linear operator

$$
\mathfrak{B}: \mathrm{H} \rightarrow \mathrm{H}^{\prime}, \quad \text { defined by }\langle\mathfrak{B} v, w\rangle=\mathfrak{b}(v, w) \quad \forall v, w \in \mathrm{H},
$$

is an isomorphism of the form

$$
\mathfrak{B}=\sum_{j=1}^{d} \mathcal{I}_{1} \otimes \cdots \otimes \mathcal{I}_{j-1} \otimes \mathfrak{B}_{j} \otimes \mathcal{I}_{j+1} \otimes \cdots \otimes \mathcal{I}_{d},
$$

which is the sum of rank-one tensor-product operators.

The central theme of the subsequent sections is to show, under suitable notions of tensorsparsity, that for $f \in \mathrm{H}^{\prime}$ the solution $u$ of the variational problem:

$$
\text { Find } u \in \mathrm{H}^{1} \text { such that } \mathfrak{b}(u, v)=\langle f, v\rangle \quad \forall v \in \mathrm{H}^{1},
$$

will inherit a certain tensor-compressibility from that of $f$. This means that for such right-hand sides $f$ the solution $u$ avoids the curse of dimensionality. 
2.2. Spectral representations and generalized Sobolev spaces. In this section, we apply well-known results on elliptic operators and spectral theory to the operator $\mathfrak{B}$ to obtain an eigensystem and to define an associated scale of Hilbert spaces that can be viewed as generalizations of classical Sobolev spaces. In fact, for specific examples of $\mathfrak{B}$ and for a certain range of smoothness scales they agree with classical Sobolev spaces (with equivalent norms). The next lemma, which we quote from $[14,15]$, is a version of the Hilbert-Schmidt theorem and will be relevant in the discussion that follows.

Lemma 1. Let $\mathcal{H}$ and $\mathcal{V}$ be separable infinite-dimensional Hilbert spaces, with $\mathcal{V}$ continuously and densely embedded in $\mathcal{H}$. Let $a: \mathcal{V} \times \mathcal{V} \rightarrow \mathbb{R}$ be a nonzero, symmetric, bounded and coercive bilinear form. Then, there exist sequences of real numbers $\left(\lambda_{n}\right)_{n \in \mathbb{N}}$ and unit $\mathcal{H}$ norm members $\left(e_{n}\right)_{n \in \mathbb{N}}$ of $\mathcal{V}$, which solve the eigenvalue problem:

$$
\text { Find } \lambda \in \mathbb{R} \text { and } e \in \mathcal{H} \backslash\{0\} \text { such that } \mathfrak{b}(e, v)=\lambda(e, v)_{\mathcal{H}} \quad \forall v \in \mathcal{V},
$$

where $(\cdot, \cdot)_{\mathcal{H}}$ signifies the inner product of $\mathcal{H}$. The $\lambda_{n}$, which can be assumed to be in increasing order with respect to $n$, are positive, bounded from below away from 0 , and $\lim _{n \rightarrow \infty} \lambda_{n}=\infty$.

Moreover, the $e_{n}$ form an $\mathcal{H}$-orthonormal system whose $\mathcal{H}$-closed span is $\mathcal{H}$ and the rescaling $e_{n} / \sqrt{\lambda_{n}}$ gives rise to a $\mathfrak{b}$-orthonormal system whose $\mathfrak{b}$-closed span is $\mathcal{V}$. Thus, we have

$$
h=\sum_{n=1}^{\infty}\left(h, e_{n}\right)_{\mathcal{H}} e_{n}, \quad\|h\|_{\mathcal{H}}^{2}=\sum_{n=1}^{\infty}\left[\left(h, e_{n}\right)_{\mathcal{H}}\right]^{2}, \quad h \in \mathcal{H}
$$

as well as

$$
v=\sum_{n=1}^{\infty} \mathfrak{b}\left(v, \frac{e_{n}}{\sqrt{\lambda_{n}}}\right) \frac{e_{n}}{\sqrt{\lambda_{n}}}, \quad\|v\|_{\mathfrak{b}}^{2}:=\mathfrak{b}(v, v)=\sum_{n=1}^{\infty}\left[\mathfrak{b}\left(v, \frac{e_{n}}{\sqrt{\lambda_{n}}}\right)\right]^{2}, \quad v \in \mathcal{V} .
$$

Furthermore, one has

$$
h \in \mathcal{H} \quad \text { and } \quad \sum_{n=1}^{\infty} \lambda_{n}\left[\left(h, e_{n}\right)_{\mathcal{H}}\right]^{2}<\infty \Longleftrightarrow h \in \mathcal{V} .
$$

Proof. The proofs of the stated results can be partially found in textbooks on functional analysis (see, for example, Theorem VI.15 in Reed \& Simon [31] or Section 4.2 in Zeidler [36]). A version of the proof for the special case in which $\mathcal{V}$ and $\mathcal{H}$ are standard Sobolev spaces is contained in Section IX.8 of Brezis [7]; using the abstract results in Chapter VI of [7], the result in Section IX.8 of [7] can be easily adapted to the setting of the present theorem. For a detailed proof we refer to Lemmas 15 and 16 in [15].

It follows from (2.1) and Lemma 1 that for each $j \in\{1, \ldots, d\}$ there exists an eigensystem $\left(e_{j, n}\right)_{n \in \mathbb{N}} \subset H_{j}$ in the factor space $H_{j}$, with the properties

$$
\left\langle e_{j, n}, e_{j, m}\right\rangle=\delta_{n, m}, \quad \mathfrak{B}_{j} e_{j, n}=\lambda_{j, n} e_{j, n}, \quad n, m \in \mathbb{N}, j=1, \ldots, d,
$$

where $\lambda_{j, n} \geq \lambda_{0}>0$ are increasing in $n$, and $\lambda_{j, n} \rightarrow \infty$ as $n \rightarrow \infty$ for each $j=1, \ldots, d$. Therefore, by $(2.7)$,

satisfy

$$
e_{\nu}:=e_{1, \nu_{1}} \otimes \cdots \otimes e_{d, \nu_{d}}, \quad \lambda_{\nu}:=\lambda_{1, \nu_{1}}+\cdots+\lambda_{d, \nu_{d}}, \quad \nu \in \mathbb{N}^{d}
$$

$$
\mathfrak{B} e_{\nu}=\lambda_{\nu} e_{\nu}, \quad \mathfrak{b}\left(\lambda_{\nu}^{-1 / 2} e_{\nu}, \lambda_{\mu}^{-1 / 2} e_{\mu}\right)=\delta_{\nu, \mu}, \quad \nu, \mu \in \mathbb{N}^{d}
$$

and hence

$$
\mathrm{e}^{-\mathfrak{B}} e_{\nu}=\mathrm{e}^{-\lambda_{\nu}} e_{\nu}=\bigotimes_{j=1}^{d} \mathrm{e}^{-\lambda_{j, \nu_{j}}} e_{j, \nu_{j}}, \quad \nu \in \mathbb{N}^{d}
$$

Since $\mathrm{H}$ is dense in $\mathrm{H}^{\prime}$, the linear span of the system of eigenfunctions $\left(e_{\nu}\right)_{\nu \in \mathbb{N}^{d}}$ is also dense in $\mathrm{H}^{\prime}$. We now define the fractional-order (generalized Sobolev) space $\mathrm{H}^{s}, s \geq 0$, as the set of all $v \in \mathrm{H}^{\prime}$ for which

$$
\|v\|_{s}^{2}:=\|v\|_{\mathrm{H}^{s}}^{2}:=\sum_{\nu \in \mathbb{N}^{d}} \lambda_{\nu}^{s}\left|\left\langle v, e_{\nu}\right\rangle\right|^{2}<\infty
$$


In particular, $\|\cdot\|_{\mathrm{H}}=\|\cdot\|_{1}$. Furthermore, when $s<0$ we define the spaces $\mathrm{H}^{s}:=\left(\mathrm{H}^{-s}\right)^{\prime}$, by duality. It is easy to see that their norms are also given by (2.17). The spaces

$$
\mathrm{H}^{s}:=\left\{v \in \mathrm{H}^{\prime}:\|v\|_{s}<\infty\right\}, \quad s \in \mathbb{R},
$$

form a scale of separable Hilbert spaces. Note further that, thanks to the orthogonality property in (2.13) and the definition of the norm (2.17), $e_{\nu} \in \mathrm{H}^{s}$ for any $\nu \in \mathbb{N}^{d}$ and any $s \in \mathbb{R}$. In classical settings, when $\mathfrak{B}$ is an elliptic differential operator, the spaces $\mathrm{H}^{s}$ agree with Sobolev spaces for a certain range of $s$, which depends on the geometry of the domain $D$ and the coefficients in the operator.

Having introduced this scale of spaces, let us note that the operator $\mathfrak{B}$, while initially defined on $\mathrm{H}$ can now be extended to all of the spaces $\mathrm{H}^{s}, s \in \mathbb{R}$ : if $v \in \mathrm{H}^{s}$, then

$$
\mathfrak{B} v:=\sum_{\nu \in \mathbb{N}^{d}} \lambda_{\nu}\left\langle v, e_{\nu}\right\rangle e_{\nu}
$$

So $\mathfrak{B}$ is an isometry between $\mathrm{H}^{t}$ and $\mathrm{H}^{t-2}$, for all $t \in \mathbb{R}$,

$$
\|\mathfrak{B} v\|_{t-2}=\|v\|_{t} .
$$

Hence, for any $t \in \mathbb{R}$, and any $f \in \mathrm{H}^{t}$, the variational problem:

$$
\text { Find } u \in \mathrm{H}^{t+2} \text { such that } \mathfrak{b}(u, v)=\langle f, v\rangle \quad \forall v \in \mathrm{H}^{-t},
$$

has a unique solution. It will be convenient to interpret (2.21) as an operator equation

$$
\mathfrak{B} u=f .
$$

The next remark records some related useful facts.

Remark 1. For any $v \in \mathrm{H}^{s}, s \in \mathbb{R}$, one has

$$
\begin{aligned}
v & =\sum_{\nu \in \mathbb{N}^{d}}\left\langle v, e_{\nu}\right\rangle e_{\nu}, \quad \text { in } \mathrm{H}^{s}, \\
\mathfrak{B}^{-1} v & =\sum_{\nu \in \mathbb{N}^{d}} \lambda_{\nu}^{-1}\left\langle v, e_{\nu}\right\rangle e_{\nu}, \quad \text { in } \mathrm{H}^{s+2}, \\
\mathrm{e}^{-\mathfrak{B}} v & =\sum_{\nu \in \mathbb{N}^{d}} \mathrm{e}^{-\lambda_{\nu}}\left\langle v, e_{\nu}\right\rangle e_{\nu}, \quad \text { in } \mathrm{H}^{s} .
\end{aligned}
$$

2.3. Rank-one tensors. We proceed to establish in this section some facts concerning rank-one tensors that will be used several times later in the paper. The following two observations relate the regularity of a rank-one tensor to the regularity of its factors.

Lemma 2. Let $s \geq 0$. A rank-one tensor $\tau=\tau_{1} \otimes \cdots \otimes \tau_{d}$ belongs to $\mathrm{H}^{s}$ if and only if $\tau_{j} \in H_{j}^{s}$, $j=1, \ldots, d$. Moreover, when $\tau \in \mathrm{H}^{s}$, there is a representation $\tau=\tau_{1} \otimes \cdots \otimes \tau_{d}$ for which

$$
\max _{j=1, \ldots, d}\left\|\tau_{j}\right\|_{H_{j}^{s}}\left(\prod_{i \neq j}\left\|\tau_{i}\right\|_{L_{2}\left(D_{i}\right)}\right) \leq\|\tau\|_{s} \leq\left(d^{\max \{0, s-1\}}\right)^{1 / 2} \sum_{j=1}^{d}\left\|\tau_{j}\right\|_{H_{j}^{s}}\left(\prod_{i \neq j}\left\|\tau_{i}\right\|_{L_{2}\left(D_{i}\right)}\right) .
$$

Proof. We begin by noting that (2.26) holds trivially for $\tau=0$. Let us therefore assume that $\tau \in \mathrm{H}^{s} \backslash\{0\}$, with $s \geq 0$. Assume first that each $\tau_{j} \in H_{j}^{s}, j=1, \ldots, d$. From the definition (2.17), we have that

$$
\begin{aligned}
\|\tau\|_{s}^{2} & =\sum_{\nu \in \mathbb{N}^{d}}\left(\lambda_{1, \nu_{1}}+\cdots+\lambda_{d, \nu_{d}}\right)^{s}\left\langle\tau_{1}, e_{1, \nu_{1}}\right\rangle^{2} \cdots\left\langle\tau_{d}, e_{d, \nu_{d}}\right\rangle^{2} \\
& \leq d^{\max \{0, s-1\}} \sum_{\nu \in \mathbb{N}^{d}}\left(\lambda_{1, \nu_{1}}^{s}+\cdots+\lambda_{d, \nu_{d}}^{s}\right)\left\langle\tau_{1}, e_{1, \nu_{1}}\right\rangle^{2} \cdots\left\langle\tau_{d}, e_{d, \nu_{d}}\right\rangle^{2} \\
& =d^{\max \{0, s-1\}} \sum_{j=1}^{d}\left(\sum_{\nu_{j}=1}^{\infty} \lambda_{j, \nu_{j}}^{s}\left\langle\tau_{j}, e_{j, \nu_{j}}\right\rangle^{2}\right)\left(\prod_{i \neq j}\left\|\tau_{i}\right\|_{0}^{2}\right)=d^{\max \{0, s-1\}} \sum_{j=1}^{d}\left\|\tau_{j}\right\|_{s}^{2}\left(\prod_{i \neq j}\left\|\tau_{i}\right\|_{0}^{2}\right) .
\end{aligned}
$$


Now, we can replace the right-hand side of the last inequality by the right-hand side of (2.26) because the $\ell_{2}$ norm does not exceed the $\ell_{1}$ norm. Thus, $\tau \in \mathrm{H}^{s}$ and we have established the second inequality in (2.26).

Concerning, the first inequality in (2.26), for any fixed $j \in\{1, \ldots, d\}$, we have

$$
\begin{aligned}
\|\tau\|_{s}^{2} & =\sum_{\nu \in \mathbb{N}^{d}}\left(\lambda_{1, \nu_{1}}+\cdots+\lambda_{d, \nu_{d}}\right)^{s}\left\langle\tau_{1}, e_{1, \nu_{1}}\right\rangle^{2} \cdots\left\langle\tau_{d}, e_{d, \nu_{d}}\right\rangle^{2} \\
& \geq \sum_{\nu \in \mathbb{N}^{d}} \lambda_{j, \nu_{j}}^{s}\left\langle\tau_{1}, e_{1, \nu_{1}}\right\rangle^{2} \cdots\left\langle\tau_{d}, e_{d, \nu_{d}}\right\rangle^{2} \\
& \geq\left\|\tau_{j}\right\|_{H_{j}^{s}}^{2}\left(\prod_{i \neq j}\left\|\tau_{i}\right\|_{0}^{2}\right)=\left\|\tau_{j}\right\|_{H_{j}^{s}}^{2}\left(\prod_{i \neq j}\left\|\tau_{i}\right\|_{L_{2}\left(D_{i}\right)}^{2}\right)
\end{aligned}
$$

which yields (2.26) for $\tau \in \mathrm{H}^{s} \backslash\{0\}$, with $s \geq 0$.

According to Lemma 2, $\tau \in \mathrm{H}^{s}$ for $s \geq 0$ if and only if $\tau \in \mathrm{H}^{s, \ldots, s}:=\otimes_{j=1}^{d} H_{j}^{s}$; for related results we refer to [33], where Besov and Sobolev spaces of dominating mixed smoothness are shown to be tensor products of Besov and Sobolev spaces of univariate functions.

We record the following consequence of the above observations in particular for later use in $\S 8$.

Corollary 1. For any $s \in \mathbb{R}$ there exists a constant $C$ such that, for any $\tau=\tau_{1} \otimes \cdots \otimes \tau_{d} \in \mathrm{H}^{-s}$,

$$
\prod_{j=1}^{d}\left\|\tau_{j}\right\|_{H_{j}^{-s}} \leq C\|\tau\|_{-s} .
$$

Proof. Since, for $s \geq 0, \mathrm{H}^{s, \ldots, s}$ is continuously embedded in $\mathrm{H}^{s}$, there exists a constant $C$ such that we have

$$
\|\tau\|_{s} \leq C\|\tau\|_{\mathrm{H}^{s, \ldots, s}}=\prod_{j=1}^{d}\left\|\tau_{j}\right\|_{H_{j}^{s}} .
$$

By duality, $\left(\mathrm{H}^{s}\right)^{\prime}=\mathrm{H}^{-s}$ is continuously embedded in $\left(\mathrm{H}^{s, \ldots, s}\right)^{\prime}$. Since

$$
\left(\mathrm{H}^{s, \ldots, s}\right)^{\prime}=\left(H_{1}^{s} \otimes \cdots \otimes H_{d}^{s}\right)^{\prime}=\left(H_{1}^{s}\right)^{\prime} \otimes \cdots \otimes\left(H_{d}^{s}\right)^{\prime}=H_{1}^{-s} \otimes \cdots \otimes H_{d}^{-s}
$$

is a tensor-product Hilbert space endowed with the corresponding cross-norm, the claim follows.

While the collection of all rank-one tensors in $\mathrm{H}^{s}$ whose $\mathrm{H}^{s}$ norm is uniformly bounded is not compact in $\mathrm{H}^{s}$, one has the following consequence of Lemma 2.

Lemma 3. For any $C>0$ and any $s^{\prime} \in \mathbb{R}$, the collection

$$
\mathcal{T}\left(s^{\prime}, C\right):=\left\{\tau=\tau_{1} \otimes \cdots \otimes \tau_{d} \in \mathrm{H}^{s^{\prime}}:\|\tau\|_{s^{\prime}} \leq C\right\}
$$

is a compact subset of $\mathrm{H}^{s}$ provided $s^{\prime}>s$.

Proof. It is easy to see (see Lemma 4) that any closed bounded ball $B$ of $\mathrm{H}^{s^{\prime}}$ is a compact subset of $\mathrm{H}^{s}$. Therefore, we only need to show that the set $\mathcal{T}\left(s^{\prime}, C\right)$ is closed in $\mathrm{H}^{s}$. Let $\tau^{(n)}$ be a sequence from this set, which converges in the topology of $\mathrm{H}^{s}$ to a function $g \in \mathrm{H}^{s}$. We need only show that $g=g_{1} \otimes \cdots \otimes g_{d}$ for some $g_{j} \in H_{j}^{s}$. If $g=0$, then clearly this limit function is in $\mathcal{T}\left(s^{\prime}, C\right)$. If $\|g\|_{s}>0$, then $\left\|\tau^{(n)}\right\|_{s} \geq C>0$ for $n$ sufficiently large.

Consider now first the case $s^{\prime}>0$. Since the norm topology of the spaces $\mathrm{H}^{s}$ gets weaker as $s$ decreases, it is sufficient to prove the Lemma in this case for $s^{\prime}>s \geq 0$. We can assume without loss of generality that for each $n$ the norms $\left\|\tau_{j}^{(n)}\right\|_{0}$ are all equal. It follows from Lemma 2 for $s^{\prime} \geq 0$, that each of the components satisfies $\left\|\tau_{j}^{(n)}\right\|_{s^{\prime}} \leq C^{\prime}$ for an absolute constant $C^{\prime}$. Hence, each of the sequences $\left(\tau_{j}^{(n)}\right)_{n \geq 1}$ is compact in $H_{j}^{s}, j=1, \ldots, d$. A common subsequence of each of them, indexed by $\left(n_{k}\right)$, converges to a limit $g_{j} \in H_{j}^{s}$, with $\left\|g_{j}\right\|_{H_{j}^{s}} \leq C^{\prime}, j=1, \ldots, d$. 
We claim that $g$ is equal to $g_{1} \otimes \cdots \otimes g_{d}$. Indeed, for any $\tau^{(n)}$, we can write

$$
\left(g_{1} \otimes \cdots \otimes g_{d}\right)-\left(\tau_{1}^{(n)} \otimes \cdots \otimes \tau_{d}^{(n)}\right)=\sum_{j=1}^{d} g_{1} \otimes \cdots \otimes g_{j-1} \otimes\left(g_{j}-\tau_{j}^{(n)}\right) \otimes \tau_{j+1}^{(n)} \otimes \cdots \otimes \tau_{d}^{(n)},
$$

with an obvious interpretation of the summands for $j \in\{1, \ldots, d\}$. Given the fact that for each of the components $g_{j}$, we have $\left\|g_{j}\right\|_{L_{2}\left(D_{j}\right)} \leq\left\|g_{j}\right\|_{H_{j}^{s}} \leq C^{\prime}$, and a similar bound for the components of the $\tau^{(n)}$, we infer from (2.26) in Lemma 2 that the $\mathrm{H}^{s}$ norm of each of the terms in the sum appearing (2.28) tends to zero as $n \rightarrow \infty$. This proves the claim for $s>0$.

When $s^{\prime} \leq 0$, we renormalize so that for each $n$, all of the norms $\left\|\tau_{j}^{(n)}\right\|_{H_{j}^{s^{\prime}}}, j=1, \ldots, d$, are equal. Then, (2.27) in Corollary 1 gives that all of these norms have a uniform bound. Hence, we again derive the existence of subsequences $\left(\tau_{j}^{(n)}\right)_{n \geq 1}$ that converge in $H_{j}^{s}$ to respective limits $g_{j}$. To show that $g$ agrees with $g_{1} \otimes \cdots \otimes g_{d}$ it suffices to prove that $\left\|g_{1} \otimes \cdots \otimes g_{d}-\tau_{1}^{(n)} \otimes \cdots \otimes \tau_{d}^{(n)}\right\|_{\mathrm{H}^{s, \ldots, s}} \rightarrow 0$, $n \rightarrow \infty$. Employing again the decomposition (2.28), this in turn, follows by using that $\|\cdot\|_{\mathrm{H}^{s, \ldots, s}}$ is a tensor product norm. That finishes the proof of the Lemma.

We now turn to the central question of this paper: Can some formulation of tensor-sparsity, or more generally tensor-compressibility, help to break the curse of dimensionality when solving the variational problems (2.8) and (2.21)?

\section{Tensor-Sparsity And Compressibility}

Numerical methods for solving partial differential equations are based on some form of approximation. In our case, we want to approximate the solution $u$ to (2.8) in the $\mathrm{H}^{1}$ norm. The simplest numerical methods utilize a sequence $\left(V_{n}\right)_{n=1}^{\infty}$ of linear spaces with $\operatorname{dim}\left(V_{n}\right) \sim n$ for the approximation. Adaptive and more advanced numerical methods replace the $V_{n}$ by nonlinear spaces $\Sigma_{n}$. Since the case of linear subspaces is subsumed by the nonlinear case, we continue our discussion in the nonlinear context. We assume in the following that the elements of $\Sigma_{n}$ are described by $\sim n$ parameters.

To understand how well a potential numerical method built on $\Sigma_{n}$ could perform, we need first to understand the approximation capabilities of $\Sigma_{n}$. The following quantification of performance will be described in a general setting of approximation in a Banach space $X$ and therefore we assume that each $\Sigma_{n} \subset X$. For any function $v \in X$, the approximation error

$$
\sigma_{n}(v)_{X}:=\inf _{g \in \Sigma_{n}}\|v-g\|_{X}
$$

tells us how well $v$ can be approximated by the elements of $\Sigma_{n}$. In the case of most interest to us, $X=\mathrm{H}^{1}, \sigma_{n}(u)_{\mathrm{H}^{1}}$ gives the optimal performance, in computing $u$, that would be possible by any numerical method based on this form of approximation. Of course, there is also the problem of constructing a numerical algorithm with this level of performance, and proving that the implementation of the algorithm can be achieved with $\mathcal{O}(n)$, or perhaps slightly more, computations.

Given a sequence $\left(\Sigma_{n}\right)_{n \geq 0}$, with $\Sigma_{0}:=\{0\}$, the approximation space $\mathcal{A}^{r}:=\mathcal{A}^{r}\left(\left(\Sigma_{n}\right)_{n \geq 0}, X\right)$ consists of all functions $v \in X$ such that

$$
\sigma_{n}(v)_{X} \leq M(n+1)^{-r}, \quad n \geq 0,
$$

and the smallest such $M$ is the norm of $v$ for the approximation space $\mathcal{A}^{r}$. More generally, we have the approximation classes $\mathcal{A}_{q}^{r}:=\mathcal{A}_{q}^{r}\left(\left(\Sigma_{n}\right)_{n \geq 0}, X\right)$, which are defined, for any $0<q \leq \infty$ and $r>0$, as the set of all $v \in X$ such that

$$
\|v\|_{\mathcal{A}_{q}^{r}\left(\left(\Sigma_{n}\right)_{n \geq 0}, X\right)}:=\left\{\begin{array}{cl}
\left(\sum_{n=0}^{\infty}\left[(n+1)^{r} \sigma_{n}(v)_{X}\right]^{q} \frac{1}{n+1}\right)^{1 / q} & \text { if } 0<q<\infty, \\
\sup _{n \geq 0}(n+1)^{r} \sigma_{n}(v)_{X}, & \text { if } q=\infty,
\end{array}\right.
$$

is finite. So, $\mathcal{A}^{r}=\mathcal{A}_{\infty}^{r}$.

When $X$ is an $L_{p}$ space or a Sobolev space, the approximation spaces for classical methods based on polynomials, splines or wavelets are well studied and are either completely characterized or very 
well understood through embeddings. They turn out to be describable by Sobolev spaces or Besov spaces (see [11]). In particular, these known results show that classical methods of approximation suffer from the curse of dimensionality. For example, membership in the approximation space $\mathcal{A}^{r}\left(\left(\Sigma_{n}\right)_{n \geq 0}, \mathrm{H}^{1}\right)$ for such methods requires the function $v$ to have $r d$ orders of smoothness in $\mathrm{H}^{1}$. As $d$ increases, this requirement will not be satisfied for typical right-hand sides $f$ in (2.8) that arise in applications. This means that numerical methods built on such classical approximation spaces are not effective when dealing with high-dimensional problems of the form (2.8).

One fairly recent viewpoint reflected by the discussion in the Introduction, and the one taken here, is that, in contrast to classical constructions, spaces $\Sigma_{n}$ built on suitable tensor formats may perform much more favorably on certain high-dimensional problems, and, indeed, break the curse of dimensionality for them. However, to the best of our knowledge no such rigorous results, in this direction, exist as of yet. In this subsection, we put forward some natural possibilities for defining sparse tensor classes $\Sigma_{n}$, subject to a budget of $\sim n$ parameters. A later subsection of this paper will show that the nonlinear spaces $\left(\Sigma_{n}\right)_{n \geq 0}$ built on these tensor constructions effectively capture the solutions to the variational problem (2.8). To show this effectiveness, one has to understand what conditions on the right-hand side $f$ guarantee that the solution $u$ is in an approximation class $\mathcal{A}^{r}\left(\left(\Sigma_{n}\right)_{n>0}, \mathrm{H}^{1}\right)$ for a reasonably large value of $r$. One can view any theorem that deduces membership of $u$ in $\mathcal{A}^{r}\left(\left(\Sigma_{n}\right)_{n>0}, \mathrm{H}^{1}\right)$ from a suitable property of $f$ as a regularity theorem for the variational problem under consideration. Such regularity results are then proved in $\S 4$.

3.1. Formulations of tensor-sparsity. In this section we introduce and compare several possible formulations of tensor-sparsity and tensor-compressibility. The main property one seeks in such sparsity classes $\Sigma_{n}$ is that the elements in $\Sigma_{n}$ should depend on $\sim n$ parameters. The common feature of these formulations is that they are based on low-dimensional eigensystems whose tensor products form a fixed background basis. In later sections we will propose an alternative way of defining tensor-sparsity, which is independent of any specific background system.

3.1.1. n-term approximation. We first discuss the well-studied nonlinear approximation procedure of $n$-term approximation from a basis $\left(\varphi_{\nu}\right)_{\nu \in \mathbb{N}^{d}}$. In our setting, we know that $\left(e_{\nu}\right)_{\nu \in \mathbb{N}^{d}}$ is a tensor basis for $\mathrm{H}^{t}, t \in \mathbb{R}$, and in particular for $\mathrm{H}^{1}$. In $n$-term approximation, the space $\Sigma_{n}$ consists of all functions $g=\sum_{\nu \in \Lambda} c_{\nu} e_{\nu}$ where $\Lambda \subset \mathbb{N}^{d}$ is a subset of indices with cardinality at most $n$. The functions in $\Sigma_{n}$ are said to be sparse of order $n$. Sometimes one places further restrictions on $\Lambda$ to ease numerical implementation and the search for the set of the best $n$ co-ordinates. For example, when $d=1$, a typical assumption is that the indices must come from the set $\left\{1, \ldots, n^{A}\right\}$ where $A$ is a fixed positive integer.

Since $\left(e_{\nu}\right)_{\nu \in \mathbb{N}^{d}}$ is also a basis for $\mathrm{H}^{t}$, the same nonlinear space $\Sigma_{n}$ can be used to approximate the right-hand side $f$ of (2.8). Let $\mathfrak{B} u=f$. If $g=\sum_{\nu \in \Lambda} c_{\nu} e_{\nu}$ is any element of $\Sigma_{n}$, then $\bar{u}:=\mathfrak{B}^{-1} g=\sum_{\nu \in \Lambda} c_{\nu} \lambda_{\nu}^{-1} e_{\nu}$ is also in $\Sigma_{n}$ and satisfies

$$
\|u-\bar{u}\|_{t+2}=\|f-g\|_{t}
$$

Therefore,

$$
\sigma_{n}(u)_{\mathrm{H}^{t+2}} \leq \sigma_{n}(f)_{\mathrm{H}^{t}}
$$

We therefore have the following simple regularity theorem: for each $r>0, f \in \mathcal{A}^{r}\left(\left(\Sigma_{n}\right)_{n \geq 0}, \mathrm{H}^{t}\right)$ implies that $u \in \mathcal{A}^{r}\left(\left(\Sigma_{n}\right)_{n \geq 0}, \mathrm{H}^{t+2}\right)$. While this is a favorable-looking result, that seems to break the curse of dimensionality, closer inspection reveals that for $u$ or $f$ to belong to the corresponding $\mathcal{A}^{r}$ space, one requires that when the coefficients in its eigenexpansion are rearranged in decreasing order the $n$-th coefficient should decay like $n^{-r-1 / 2}$. This in turn is like a smoothness condition of order $r d$ placed on $f$ and $u$. Hence, the conditions on the data $f$ become more and more restrictive when the dimension $d$ increases.

3.1.2. Variable rank-one tensors. Our next sparsity model again draws on the eigensystem $\left(e_{\nu}\right)_{\nu \in \mathbb{N}^{d}}$ as a reference basis but now employs low-dimensional eigenbases to parametrize variable rank-one tensors. More precisely, let us first consider, for any $j \in\{1, \ldots, d\}$, the eigenbasis $\left(e_{j, k}\right)_{k=1}^{\infty}$ associated with the low-dimensional operator $\mathfrak{B}_{j}$ on $H_{j}$. The $e_{j, k}, k=1,2, \ldots$, are functions that depend only on $x_{j}$. For each $j \in\{1, \ldots, d\}$, let $\Sigma_{m}^{j}$ be the collection of all linear combinations of 
at most $m$ of the $j$ th eigensystem elements $e_{j, k}$. If we fix a value of $j \in\{1, \ldots, d\}$, then the space $\Sigma_{m}^{j}$ is the space of $m$-sparse functions for this basis. Thus, any function $g_{j} \in \Sigma_{m}^{j}$ can be written as

$$
g_{j}=\sum_{k \in \Gamma_{j}} c_{k} e_{j, k}
$$

where $\#\left(\Gamma_{j}\right) \leq m$.

Next consider any $\mathbf{m}=\left(m_{1}, \ldots, m_{d}\right)$ and functions $g_{j} \in \Sigma_{m_{j}}^{j}, j=1, \ldots, d$. Then, the functions

$$
g=\bigotimes_{j=1}^{d} g_{j}
$$

are rank-one tensors, which depend only on the $|\mathbf{m}|:=m_{1}+\cdots+m_{d}$ positions $\Gamma_{j}, j=1, \ldots, d$, and the $|\mathbf{m}|$ coefficients of the $g_{j}$. We define $\Sigma_{\mathbf{m}}$ to be the set of all such functions $g$.

Note that if $g \in \Sigma_{\mathbf{m}}$ is expanded in terms of the eigenbasis $\left(e_{\nu}\right)_{\nu \in \mathbb{N}^{d}}$, it would involve $m_{1} \cdots m_{d}$ terms. However, what is key is that the coefficients in the eigenbasis expansion of $g$ only depend — in a highly nonlinear fashion — on $2|\mathbf{m}|$ parameters, namely the $|\mathbf{m}|$ indices in the sets $\Gamma_{j}$ and the $|\mathbf{m}|$ corresponding coefficients that appear in $g_{j}, j=1, \ldots, d$.

In order to have a more compact notation for these functions $g$ in what follows, we introduce $\Gamma:=\Gamma_{1} \times \cdots \times \Gamma_{d}$, which is the set of indices appearing in $g$ and $C=C_{g}$ is the rank-one tensor of coefficients

We also use the notation

$$
C=C_{g}:=\left(c_{\nu_{1}, 1} \cdots c_{\nu_{d}, d}\right)_{\nu \in \Gamma}
$$

$$
T_{\Gamma, C}=g
$$

when we want to explicitly indicate for a $g \in \Sigma_{\mathbf{m}}$ the index set $\Gamma$ and the tensor of coefficients $C$

We define the sparsity space $\Sigma_{n}$ as the set of all functions

$$
g=\sum_{k=1}^{s} g_{k}, \quad g_{k} \in \Sigma_{\mathbf{m}_{k}}, \quad \sum_{k=1}^{s}\left|\mathbf{m}_{k}\right| \leq n,
$$

and introduce, for any $v \in \mathrm{H}^{t}$, the approximation error

$$
\sigma_{n}\left(v, \mathrm{H}^{t}\right):=\inf _{g \in \Sigma_{n}}\|v-g\|_{t} .
$$

Obviously a function in $\Sigma_{n}$ depends on at most $n$ positions and $n$ coefficients, so it can be viewed as an analogue of $n$-term approximation except that it strongly exploits tensor structure. Indeed, if such a function were expanded into the tensor basis $\left(e_{\nu}\right)_{\nu \in \mathbb{N}^{d}}$, it would possibly require $(n / d)^{d}$ terms. Note that this definition of $\Sigma_{n}$ includes all of the $n$-term functions of $§ 3.1 .1$.

3.1.3. Restricted index sets. The space $\Sigma_{n}$ as defined by (3.10) is not suitable for use in numerical computations. One reason for this is that there is no control on the index sets appearing in the $\Gamma_{j}$. This can be circumvented in applications by placing restrictions on the set of indices similar to the restrictions in $n$-term approximation already described. To make all of this formal, we suppose that for each $m \geq 1$ and $j \in\{1, \ldots, d\}$, we have a finite set $\mathcal{R}_{m, j} \subset \mathbb{N}$. We will require that for any given $\mathbf{m}$, the sets $\Gamma_{j}$ are subsets of $\mathcal{R}_{m_{j}, j}$ for each $j=1, \ldots, d$, or, in other words, that $\Gamma \subset \mathcal{R}_{\mathbf{m}}$ where $\mathcal{R}_{\mathbf{m}}:=\mathcal{R}_{m_{1}, 1} \times \cdots \times \mathcal{R}_{m_{d}, d}$. The typical choice for the restriction sets $\mathcal{R}_{m, j}$ is $\mathcal{R}_{m, j}:=\left\{1, \ldots, m^{A}\right\}$ where $A$ is a fixed integer. Such choices are independent of $j$. In what follows, if we wish to indicate the difference between $\Sigma_{n}$ and the space defined with restrictions, we will denote the latter by $\Sigma_{n}(\mathcal{R})$.

One possible restriction, certainly a strong one, is that the sets $\mathcal{R}_{m, j}$ are all one and the same, and equal to $\{1, \ldots, m\}$ for each $j=1, \ldots, d$. Notice that in that case only the $e_{\nu}$ with $\|\nu\|_{\ell_{\infty}} \leq m$ are available to be used. Thus the component spaces $\Sigma_{m_{j}}^{j}$ are now linear spaces, however the spaces $\Sigma_{\mathbf{m}}$ and $\Sigma_{n}$ are not linear spaces.

For later use we record in the following lemma a simple sufficient condition for a rank-one tensor to be in the approximation space $\mathcal{A}^{r}$. We shall suppose that we are measuring the error in the norm of $\mathrm{H}^{t}$. 
Lemma 4. Suppose that $\mathbf{m}=(m, \ldots, m)$. Consider the above case of restricted approximation when $\mathcal{R}_{\mathbf{m}}$ contains $\{1, \ldots, m\}^{d}$. Then, for any $\delta>0$ and any rank-one tensor function $v(x)=$ $v_{1}\left(x_{1}\right) \cdots v\left(x_{d}\right)$ in $\mathrm{H}^{t+\delta}$, we have that

$$
\inf _{g \in \Sigma_{\mathbf{m}}}\|v-g\|_{t} \leq\left[\lambda_{m}^{*}\right]^{-\delta / 2}\|v\|_{t+\delta}
$$

where

$$
\lambda_{m}^{*}:=\min _{1 \leq j \leq d} \lambda_{j, m+1}
$$

Proof. As has been already noted, the function $g:=\sum_{\nu \leq \mathbf{m}}\left\langle v, e_{\nu}\right\rangle e_{\nu}$ is in $\Sigma_{\mathbf{m}}$. If $\nu \not \leq \mathbf{m}$, then, for some $j \in\{1, \ldots, d\}$, we have $\nu_{j} \geq m+1$ and

$$
\lambda_{\nu} \geq \lambda_{j, m+1} \geq \lambda_{m}^{*}
$$

Therefore, from (2.17) we have

$$
\|v-g\|_{t}^{2}=\sum_{\nu \geq \mathbf{m}} \lambda_{\nu}^{t+\delta} \lambda_{\nu}^{-\delta}\left\langle v, e_{\nu}\right\rangle^{2} \leq\left[\lambda_{m}^{*}\right]^{-\delta} \sum_{\nu>\mathbf{m}} \lambda_{\nu}^{t+\delta}\left\langle v, e_{\nu}\right\rangle^{2} \leq\left[\lambda_{m}^{*}\right]^{-\delta}\|v\|_{t+\delta}^{2}
$$

This gives (3.12).

As a simple example of the above result, we take the $\mathfrak{B}_{j}$ to be the Laplacian on a Lipschitz domain $D_{j} \subset \mathbb{R}^{2}$, with homogeneous Dirichlet boundary condition on $\partial D_{j}, j=1, \ldots, d$. Then the eigenvalues $\lambda_{j, k}$ grow like $k$ and $\lambda_{m}^{*} \approx m$. Hence the approximation rate for the $v$ in the lemma is of order $m^{-\delta / 2}$. Thus for an investment of computing $d m$ coefficients, we obtain accuracy $m^{-\delta}$. For an analogous result on the spectral asymptotics of degenerate elliptic operators that arise in the context of Fokker-Planck equations, we refer to [14].

3.1.4. Other bases for rank-one sparse decompositions. The spaces $\Sigma_{n}$ and their restricted counterparts $\Sigma_{n}(\mathcal{R})$ may still be inappropriate for numerical implementation (except perhaps in the case of Fourier spectral methods) because they require the computation of the eigenvalues and eigenfunctions of the elliptic operator under consideration. For this reason, numerical algorithms use other readily available tensor bases $\left(\varphi_{\nu}\right)_{\nu \in \mathbb{N}^{d}}$, such as wavelet or spline bases. In this case, the role of $e_{\nu}$ is replaced by $\varphi_{\nu}, \nu \in \mathbb{N}^{d}$, in the definition of $\Sigma_{n}$ and $\sigma_{n}$. In general, different bases give different approximation classes $\mathcal{A}^{r}$ and these classes should be viewed as ways of measuring smoothness. Sometimes, it is possible to prove for some choices of bases that the approximation classes are the same. For example, this is the case for univariate $n$-term approximation using wavelet and spline bases. We do not wish, in this paper, to enter too deeply into this issue since it depends heavily on the properties of $D$ and the constructed bases. For the construction and analysis of algorithms using such background bases we refer to [1, 2].

\section{REgUlarity THEOREMS BASED ON TENSOR-SPARSITY: DATA WITH LOW REGULARITY}

We now turn to proving regularity theorems in terms of the approximation classes based on the tensor systems $\left(\Sigma_{n}\right)_{n \geq 0}$ introduced in $§ 3.1 .2$. We shall prove that if the right-hand side $f$ is in an approximation class $\mathcal{A}^{\alpha}\left(\left(\Sigma_{n}\right)_{n \geq 0}, \mathrm{H}^{t}\right)$ for some $\alpha>0$, then the solution $u$ of the variational problem:

$$
\text { Find } u \in \mathrm{H}^{t+2} \text { such that } \mathfrak{b}(u, v)=\langle f, v\rangle \quad \forall v \in \mathrm{H}^{-t} \text {, }
$$

belongs to the approximation class $\mathcal{A}^{\alpha^{\prime}}\left(\left(\Sigma_{n}\right)_{n \geq 0}, \mathrm{H}^{t+2}\right)$ for all $\alpha^{\prime} \in(0, \alpha)$. To prepare for the proof of this result, we begin with the following lemma.

Lemma 5. Let $G(x):=1 / x, x>0$, and fix any $\beta>0$. For each integer $r \geq 1$, there exists a function

$$
S_{r}(x):=\sum_{k=1}^{r} \omega_{k} \mathrm{e}^{-\alpha_{k} x}, \quad x>0,
$$

with $\alpha_{k}=\alpha_{r, k}>0, \omega_{k}=\omega_{r, k}>0, k=1, \ldots, r$, such that 
(i) we have the error bound

$$
\left\|G-S_{r}\right\|_{L_{\infty}[\beta, \infty)} \leq \frac{16}{\beta} \mathrm{e}^{-\pi \sqrt{r}}
$$

(ii) in addition,

$$
S_{r}(x) \leq \frac{1}{x} \quad \text { for all } x \geq \frac{1}{8} \mathrm{e}^{\pi \sqrt{r}} .
$$

Proof. This follows from the results in [5, 6] and we only sketch the argument therein. The starting point is that $G$ is a completely monotone function. Defining the class of exponential sums

$$
\mathcal{E}_{r}:=\left\{\sum_{k=1}^{r} \omega_{k} \mathrm{e}^{-\alpha_{k} x}: x>0, \alpha_{k}>0, \omega_{k} \in \mathbb{R}\right\},
$$

it can be shown that for any bounded closed interval $[1, R], R>1$, the function

$$
S_{r, R}(x)=\sum_{k=1}^{r} \omega_{r, k, R} \mathrm{e}^{-\alpha_{r, k, R} x}
$$

from $\mathcal{E}_{r}$ that minimizes

$$
E_{r,[1, R]}(G):=\inf \left\{\|G-S\|_{L^{\infty}[1, R]}: S \in \mathcal{E}_{r}\right\}
$$

exists, is unique, and is characterized by an alternation property, from which one deduces that

$$
0 \leq S_{r, R}(x) \leq \frac{1}{x}, \quad x \geq R
$$

The following estimate for the error is proved in [5]:

$$
E_{r,[1, R]}(G)=\left\|G-S_{r, R}\right\|_{L_{\infty}[1, R]} \leq 16 \mathrm{e}^{-\frac{\pi^{2} r}{\log (8 R)}} .
$$

In order to obtain a bound on the whole real line one uses that $G$ decays and aims to minimize $\left.\max \left\{E_{r,[1, R]}, 1 / R\right)\right\}$ over all choices $R>1$. Taking $\left.R=R_{r}:=\frac{1}{8} \exp (\pi \sqrt{r})\right)$, gives

$$
E_{r,\left[1, R_{r}\right]}(G) \leq 16 \mathrm{e}^{-\pi \sqrt{r}} .
$$

Because of (4.6) we can replace the interval $\left[1, R_{r}\right]$ in $(4.8)$ by $[1, \infty)$. This proves (4.3) and (4.4) with $S_{r}:=S_{r, R_{r}}$ for the case $\beta=1$. The case of general $\beta$ follows from a simple rescaling. The positivity of the weights $\omega_{r, k, R}$ in (4.5) is known to hold in general for best exponential sum approximations to completely monotone functions, see [4], and that then implies the desired positivity of the $\omega_{r, k}:=\omega_{r, k, R_{r}}$.

The following lemma gives a similar result but with an added restriction on the coefficients $\alpha_{k}$.

Lemma 6. Let $G(x):=1 / x, x>0$, and fix any $\beta>0$. For each integer $r \geq 1$, there exists a function

$$
\bar{S}_{r}(x):=\sum_{k=1}^{r} \bar{\omega}_{k} \mathrm{e}^{-\alpha_{k} x}, \quad x>0,
$$

with $\alpha_{k}=\alpha_{r, k}>0$ and $\bar{\omega}_{k}=\omega_{r, k} \geq 0, k=1, \ldots, r$, such that the following hold.

(i) Whenever $\bar{\omega}_{k}>0$, we have $\alpha_{k} \geq T_{r}^{-1}$, where $\left.T_{r}:=\frac{1}{8} \exp (\pi \sqrt{r})\right), k=1, \ldots, r$.

(ii) We have the error bound

$$
\left\|G-\bar{S}_{r}\right\|_{L_{\infty}[\beta, \infty)} \leq\left(\frac{16}{\beta}+8 r \mathrm{e}\right) \mathrm{e}^{-\pi \sqrt{r}} .
$$

(iii) In addition,

$$
\bar{S}_{r}(x) \leq \frac{1}{x} \quad \text { for all } x \geq \frac{1}{8} \mathrm{e}^{\pi \sqrt{r}}
$$


Proof. We start with the function $S_{r}=\sum_{k=1}^{r} \omega_{k} \mathrm{e}^{-\alpha_{k} x}$ of the previous lemma with $\alpha_{k}>0$ and $\omega_{k}>0$ for all $k \in\{1, \ldots, r\}$. Suppose that for some $k \in\{1, \ldots, r\}$ we have $\alpha_{k} \leq T_{r}^{-1}$. Then, $\mathrm{e}^{-1} \leq \mathrm{e}^{-\alpha_{k} T_{r}}$ and therefore $\omega_{k} \mathrm{e}^{-1} \leq \omega_{k} \mathrm{e}^{-\alpha_{k} T_{r}}$. In view of (ii) of the previous lemma with $x=T_{r}$, we have $\omega_{k} \mathrm{e}^{-1} \leq \omega_{k} \mathrm{e}^{-\alpha_{k} T_{r}} \leq T_{r}^{-1}$, and thus also $\omega_{k} \leq \mathrm{e} T_{r}^{-1}$. Hence, if for each such $\alpha_{k}$, we set $\bar{\omega}_{k}:=0$ and define $\bar{\omega}_{k}=\omega_{k}$ for all other $k \in\{1, \ldots, r\}$, we obtain a new function $\bar{S}_{r}$ defined by $\bar{S}_{r}(x):=\sum_{k=1}^{r} \bar{\omega}_{k} \mathrm{e}^{-\alpha_{k} x}, x>0$, such that

$$
\bar{S}_{r}(x) \leq S_{r}(x) \leq \bar{S}_{r}(x)+r \mathrm{e} T_{r}^{-1}=\bar{S}_{r}(x)+8 r \mathrm{e}^{-\pi \sqrt{r}} \quad \text { for all } x>0 .
$$

Part (ii) then follows from (4.12) and part (i) of Lemma 5 via the triangle inequality, while part (i) follows directly from the definition of $\bar{\omega}_{k}$.

All of the remaining claims of the lemma follow from (4.12) together with the corresponding statement of Lemma 5 .

Exponential sums such as the one in Lemma 5 have been considered in several works for the purpose of constructing low-rank approximations to inverses of finite-dimensional linear operators (i.e., matrices); see e.g. $[18,16]$. However, in the finite-dimensional setting the metrics for the domain and the range of the linear operators were taken to be the same: typically the Euclidean metric. When the discrete operator is to approximate an infinite-dimensional operator, such as a differential operator, with different domain and range topologies (e.g. $\mathrm{H}^{1}$ and $\mathrm{H}^{-1}$ ), the discrete operator becomes more and more ill-conditioned when the discretization is refined. As a consequence, the accuracy of an approximate discrete solution cannot be well estimated by the corresponding discrete residual. The deviation of the corresponding expansion in a "problemrelevant" function space norm is in general not clear. Therefore, the fully discrete approach does not immediately provide a rigorous rate distortion result in the continuous PDE setting.

Expanding further on the above point, note that the operator $\mathfrak{B}$ is an isomorphism only as a mapping between spaces of different regularity levels that are not endowed with tensor product norms. A rigorous assessment of the error has to take this mapping property into account. That, however, has an adverse effect on tensor sparsity because the representation of $\mathfrak{B}^{-1}$ in the eigenbasis is a diagonal operator of infinite rank since the diagonal entries $\lambda_{\nu}^{-1}$ are not separable, see also [2]. More importantly, as will be seen next, the actual ranks required to approximate $u$ to within a given tolerance in $\|\cdot\|_{s}$, for $f$ measured in $\|\cdot\|_{t}$, say, strongly depends on the corresponding "regularity shift" $s-t$. Properly taking such "regularity shifts" into account in the course of low-rank approximation is a central issue in the subsequent developments of this paper.

The following result is our first main regularity theorem. It will be convenient to define the smallest eigenvalue of $\mathfrak{B}$ :

$$
\underline{\lambda}:=\min _{\nu \in \mathbb{N}^{d}} \lambda_{\nu}=\lambda_{(1, \ldots, 1)}
$$

Theorem 1. Suppose that $t \in \mathbb{R}$ and that $f \in \mathcal{A}^{\alpha}\left(\left(\Sigma_{n}\right)_{n \geq 0}, \mathrm{H}^{t}\right), \alpha>0$; then, the weak solution $u \in \mathrm{H}^{t+2}$ to (2.21) satisfies the following inequality:

$$
\sigma_{n[\log n]^{2}}(u)_{\mathrm{H}^{t+2}} \leq A\|f\|_{\mathcal{A}^{\alpha}\left(\left(\Sigma_{n}\right)_{n \geq 0}, \mathrm{H}^{t}\right)} n^{-\alpha}, \quad n=1,2, \ldots,
$$

where the constant $A$ depends only on $\alpha$ and the smallest eigenvalue $\underline{\lambda}>0$ of $\mathfrak{B}$. In particular, $u$ belongs to $\mathcal{A}^{\alpha^{\prime}}\left(\left(\Sigma_{n}\right)_{n \geq 0}, \mathrm{H}^{t+2}\right)$ for all $\alpha^{\prime} \in(0, \alpha)$.

Proof. We fix $n \geq 1$. The assumption that $f \in \mathcal{A}^{\alpha}\left(\left(\Sigma_{n}\right)_{n \geq 0}, \mathrm{H}^{t}\right)$ implies the existence of a $g_{n} \in \Sigma_{n}$ such that

$$
\left\|f-g_{n}\right\|_{t} \leq M n^{-\alpha}, \quad M:=\|f\|_{\mathcal{A}^{\alpha}\left(\left(\Sigma_{n}\right)_{n \geq 0}, \mathrm{H}^{t}\right)} .
$$

Membership in $\Sigma_{n}$ means that $g_{n}$ is of the form

$$
g_{n}:=\sum_{j=1}^{k} h_{j}, \quad h_{j}:=T_{\Lambda(j), C(j)},
$$

where

$$
\Lambda(j)=\Lambda_{1}(j) \times \cdots \times \Lambda_{d}(j)
$$


and

$$
C(j)=\mathbf{c}_{1}(j) \otimes \cdots \otimes \mathbf{c}_{d}(j) .
$$

Here, each $\mathbf{c}_{i}(j)$ is a vector in $\mathbb{R}^{m_{i}(j)}$, where $m_{i}(j):=\#\left(\Lambda_{i}(j)\right)$. We know that the mode sizes $m(j)=\sum_{i=1}^{d} m_{i}(j)=\sum_{i=1}^{d} \#\left(\Lambda_{i}(j)\right)$ of the tensors $C(j)$ satisfy

$$
2 \sum_{j=1}^{k} m(j) \leq n
$$

Consider now $\bar{u}_{n}=\mathfrak{B}^{-1} g_{n}$. We also know that

$$
\left\|u-\bar{u}_{n}\right\|_{t+2}=\left\|f-g_{n}\right\|_{t} \leq M n^{-\alpha}, \quad M:=\|f\|_{\mathcal{A}^{\alpha}\left(\left(\Sigma_{n}\right)_{n \geq 0}, \mathrm{H}^{t}\right)} .
$$

We deduce from (4.16) that

$$
\bar{u}_{n}=\sum_{j=1}^{k} \bar{h}_{j}, \quad \bar{h}_{j}:=\mathfrak{B}^{-1} h_{j} .
$$

We will next show how each of the functions $\bar{h}_{j}$ can be well approximated by functions from $\Sigma_{n}$. To this end, we fix $j$ and write

$$
h_{j}=\sum_{\nu \in \Lambda(j)} c_{\nu}(j) e_{\nu}
$$

so that

$$
\bar{h}_{j}=\sum_{\nu \in \Lambda(j)} \lambda_{\nu}^{-1} c_{\nu}(j) e_{\nu}
$$

where, of course, the $c_{\nu}(j)$ are determined by the at most $n / 2$ parameters defining the factors in (4.18). While, for a fixed $j$, the $c_{\nu}(j)$ form a rank-one tensor, $\lambda_{\nu}^{-1}$ is not separable so that $\bar{h}_{j}$ has infinite rank. To obtain low-rank approximations to $\bar{h}_{j}$ we use Lemma 5 , where $\underline{\lambda}>0$ is the smallest of the eigenvalues $\lambda_{\nu}$ of $\mathfrak{B}$, and approximate $\lambda_{\nu}^{-1}$ by an exponential sum $S_{r}\left(\lambda_{\nu}\right)$ which is a sum of $r$ separable terms.

In fact, using $S_{r}$ as defined in Lemma 5 , with $r$ to be chosen momentarily, we define

$$
\hat{h}_{j}:=\sum_{\nu} S_{r}\left(\lambda_{\nu}\right) c_{\nu}(j) e_{\nu},
$$

which can be rewritten as

$$
\hat{h}_{j}=\sum_{k=1}^{r} \omega_{k}\left\{\sum_{\nu \in \Lambda(j)}\left[\mathrm{e}^{-\alpha_{k} \lambda_{\nu}} c_{\nu}(j)\right] e_{\nu}\right\} .
$$

We then define $\hat{u}_{n}=\sum_{j=1}^{k} \hat{h}_{j}$.

From the tensor structure of $h_{j}$ (see (4.16)) and the fact that $\mathrm{e}^{-\alpha_{k} \lambda_{\nu}}=\mathrm{e}^{-\alpha_{k} \lambda_{1, \nu_{1}}} \cdots \mathrm{e}^{-\alpha_{k} \lambda_{d, \nu_{d}}}$ is separable, we see that each of the functions

$$
\sum_{\nu \in \Lambda(j)} \mathrm{e}^{-\alpha_{k} \lambda_{\nu}} c_{\nu}(j) e_{\nu}, \quad k=1, \ldots, r,
$$

is in $\Sigma_{\mathbf{m}(j)}$. Since, for each $j$, there are $r$ such functions, we deduce that $\hat{u}_{n}$ is in $\Sigma_{r n}$.

Writing $\bar{u}_{n}=\sum_{\nu} \lambda_{\nu}^{-1} a_{\nu} e_{\nu}$ for a suitable sequence $\left(a_{\nu}\right)_{\nu \in \cup_{j=1}^{k} \Lambda(j)}$, we have, by definition, that

$$
\hat{u}_{n}=\sum_{\nu} S_{r}\left(\lambda_{\nu}\right) a_{\nu} e_{\nu}
$$

We can now bound $\left\|\bar{u}_{n}-\hat{u}_{n}\right\|_{t}$ as follows. With $A_{0}:=16 / \underline{\lambda}$, we obtain

$$
\begin{aligned}
\left\|\bar{u}_{n}-\hat{u}_{n}\right\|_{t+2}^{2} & =\left\|\sum_{\nu}\left[\lambda_{\nu}^{-1}-S_{r}\left(\lambda_{\nu}\right)\right] a_{\nu} e_{\nu}\right\|_{t+2}^{2}=\sum_{\nu} \lambda_{\nu}\left[\left[\lambda_{\nu}^{-1}-S_{r}\left(\lambda_{\nu}\right)\right] a_{\nu}\right]^{2} \\
& \leq\left[A_{0} \mathrm{e}^{-\pi \sqrt{r}}\right]^{2} \sum_{\nu} \lambda_{\nu} a_{\nu}^{2}=\left[A_{0} \mathrm{e}^{-\pi \sqrt{r}}\right]^{2}\left\|\bar{u}_{n}\right\|_{t+2}^{2}=\left[A_{0} \mathrm{e}^{-\pi \sqrt{r}}\right]^{2}\left\|g_{n}\right\|_{t}^{2} \\
& \leq\left[A_{0} \mathrm{e}^{-\pi \sqrt{r}}\right]^{2}\|f\|_{t}^{2} .
\end{aligned}
$$


We now take $r$ as the smallest integer for which $\pi \sqrt{r} \geq \alpha \log n$. For this choice of $r$, we obtain

$$
\left\|\bar{u}_{n}-\hat{u}_{n}\right\|_{t+2} \leq A_{0}\|f\|_{t} n^{-\alpha} .
$$

When this is combined with (4.20) by a triangle inequality in the $\mathrm{H}^{t+2}$ norm, we obtain

$$
\left\|u-\hat{u}_{n}\right\|_{t+2} \leq M n^{-\alpha}+A_{0}\|f\|_{-t} n^{-\alpha} \leq\left(A_{0}+1\right)\|f\|_{\mathcal{A}^{\alpha}\left(\left(\Sigma_{n}\right)_{n \geq 0}, \mathrm{H}^{t}\right)} n^{-\alpha} .
$$

Since $\hat{u}_{n}$ is in $\Sigma_{r n}$ and $r \sim C_{\alpha}[\log n]^{2}$, where $C_{\alpha}$ is a positive constant dependent solely on $\alpha$, by absorbing the constants $A_{0}+1$ and $C_{\alpha}$ into the definition of the constant $A=A(\alpha, \underline{\lambda})$, we deduce (4.14). That completes the proof.

Suppose that in place of $\Sigma_{n}$ we use the spaces $\Sigma_{n}(\mathcal{R})$ with restrictions on the indices of the $e_{\nu}$ as described in $\S 3.1 .3$. The proof of the last theorem then applies verbatim with these restrictions. Hence, we have the following result.

Theorem 2. Suppose that $t \in \mathbb{R}$ and $\left.f \in \mathcal{A}^{\alpha}\left(\Sigma_{n}(\mathcal{R})\right)_{n \geq 0}, \mathrm{H}^{t}\right), \alpha>0$; then, the corresponding solution $u \in \mathrm{H}^{t+2}$ to (2.21) satisfies the following inequality:

$$
\sigma_{n[\log n]^{2}}(u)_{\mathrm{H}^{t+2}} \leq A\|f\|_{\mathcal{A}^{\alpha}\left(\left(\Sigma_{n}(\mathbb{R})\right)_{n \geq 0}, \mathrm{H}^{t}\right)} n^{-\alpha}, \quad n=1,2, \ldots,
$$

where the constant $A$ depends only on $\alpha$ and the smallest eigenvalue $\underline{\lambda}>0$ of $\mathfrak{B}$. In particular, $u$ belongs to $\mathcal{A}^{\alpha^{\prime}}\left(\left(\Sigma_{n}(\mathcal{R})\right)_{n \geq 0}, \mathrm{H}^{t+2}\right)$ for all $\alpha^{\prime} \in(0, \alpha)$.

We have formulated the above results in terms of the approximation spaces $\mathcal{A}^{\alpha}$. This only gives information for polynomial order decay. We can work in more generality. Let $\gamma$ be a strictly monotonically increasing function defined on $[0, \infty)$, with $\gamma(0)>0$. We define the approximation class $\mathcal{A}^{\gamma}\left(\left(\Sigma_{n}\right)_{n \geq 0}, \mathrm{H}^{t}\right)$ as the set of all $v \in \mathrm{H}^{t}$ such that

$$
\gamma(n) \sigma_{n}(f)_{\mathrm{H}^{t}} \leq M, \quad n \geq 0,
$$

with (quasi-) norm defined as the smallest such $M$. We then have the following theorem.

Theorem 3. Suppose that $t \in \mathbb{R}$ and that $f \in \mathcal{A}^{\gamma}\left(\left(\Sigma_{n}\right)_{n>0}, \mathrm{H}^{t}\right)$; then, the corresponding solution $u \in \mathrm{H}^{t+2}$ to (2.21) satisfies the following inequality:

$$
\sigma_{n[\log \gamma(n)]^{2}}(u)_{\mathrm{H}^{t+2}} \leq A\|f\|_{\mathcal{A}^{\gamma}\left(\left(\Sigma_{n}(\mathbb{R})\right)_{n \geq 0}, \mathrm{H}^{t}\right)}[\gamma(n)]^{-1}, \quad n=1,2, \ldots,
$$

where the constant $A$ depends only on the smallest eigenvalue $\underline{\lambda}>0$ of $\mathfrak{B}$. In particular, $u$ belongs to $\mathcal{A}^{\bar{\gamma}}\left(\left(\Sigma_{n}\right)_{n \geq 0}, \mathrm{H}^{t+2}\right)$, where $\bar{\gamma}(x):=\gamma\left(G^{-1}(x)\right)$ with $G(x):=x[\log \gamma(x)]^{2}, x>0$.

Proof. The proof is in essence the same as that of Theorem 1 with $n^{\alpha}$ replaced by $\gamma(n)$, and $r \geq 1$ chosen so that $\pi \sqrt{r} \geq \log \gamma(n)$. This gives (4.32) from which the remaining claim easily follows upon observing that (4.32) just means $\sigma_{G(n)}(u)_{\mathrm{H}^{t+2}} \leq A\|f\|_{\mathcal{A}^{\gamma}\left(\left(\Sigma_{n}(\mathbb{R})\right)_{n>0}, \mathrm{H}^{t}\right)}[\gamma(n)]^{-1}$, and putting $m=G(n), n=G^{-1}(m)$.

Remark 2. Let us remark on how the curse of dimensionality enters into Theorem 3. This theorem says that for $f \in \mathcal{A}^{\gamma}\left(\left(\Sigma_{n}\right)_{n \geq 0}, \mathrm{H}^{t}\right)$ the number of degrees of freedom needed to approximate the solution $u$ in $\mathrm{H}^{t+2}$ to within accuracy $\varepsilon$ is of the order $\bar{\gamma}^{-1}\left(A\|f\|_{\mathcal{A}^{\gamma}} / \varepsilon\right)$ where $A$ is a fixed constant independent of the spatial dimension d. Moreover, the approximations to $u$ are derived from nonlinear information on the data $f$ given in terms of low-rank approximations. Hence, under this assumption on the data the curse of dimensionality is broken. However, the regularity assumption on $f$ depends on $d$ because the larger $d$ the fewer degrees of freedom can be spent on each tensor factor. However, as addressed in more detail later, when the tensor factors approximate functions having a certain (low-dimensional) Sobolev or Besov regularity the deterioration of accuracy when $d$ grows is expected to be at most algebraic in d so that one can, in principle, still assert tractability in the sense that the computational work needed in order to realize a given target accuracy does not depend exponentially on $d$. 


\section{A Basis-Free Tensor-Sparsity Model}

The primary purpose of the sparsity model considered thus far is to highlight in a technically simple fashion, on the one hand, the principal mechanism of how tensor-sparsity of the data is inherited by the solution and, on the other hand, the relevance of the structure of the spectrum of elliptic operators in this context. While this is an interesting theoretical result, it does not lead directly to a numerical method that exploits this compressibility in an efficient numerical algorithm. There are several reasons for this.

The first of these is the fact that, in general, the eigenfunction basis is not available to us, and computing even the first few eigenfunctions will generally require significant computational effort. Moreover, even, if we had computed the eigenfunction basis and the representation of $f$ in this basis, it is not a simple task to find a good low-rank approximation $g$ to $f$.

A second point is that in the classical setting of differential operators, the assumption that $f$ is compressible in the eigenfunction basis may in fact exclude rather simple functions. Consider, for example, the Poisson equation subject to a homogeneous Dirichlet boundary condition:

$$
-\Delta u=1 \quad \text { in } \quad D=(0,1)^{d}, \quad u=0 \quad \text { on } \quad \partial D .
$$

In this case one has $e_{\nu}(x)=2^{-d / 2} \prod_{j=1}^{d} \sin \left(\pi \nu_{j} x_{j}\right), \nu \in \mathbb{N}^{d}$. Note that the right-hand side $f(x) \equiv 1$ is a rank-one function. However, its expansion in terms of the $e_{\nu}$ has infinitely many terms. Because of the boundary conditions, parametrizing the solution and the right-hand side with respect to the same basis may not be the most favorable approach. In fact, each univariate factor 1 of $f$ has an infinite expansion in the univariate basis $\left(\sin \left(\pi k x_{j}\right)\right)_{k \in \mathbb{N}}$. Thus we shall next propose a way of describing tensor-sparsity without referring to any particular background basis and where the corresponding regularity notion does not become stronger when $d$ increases.

Perhaps, the main shortcoming of the idea of sparsity introduced in the previous sections is that the tensors that arise in the approximation lack stability. Indeed, we have no control on how well the approximants $g$ can themselves be numerically resolved. Typically, the computability of $g$ is related to its regularity, for example, in the family of spaces $\mathrm{H}^{s}$.

The purpose of this section is to remedy some of these deficiencies. This will be accomplished by putting forward a form of tensor sparsity and compressibility that, on the one hand, does not depend on the eigenfunction basis and, in fact, is independent of any basis, and on the other hand, it imposes a regularity on the approximates $g$ to $f$, thereby making them computable. Moreover, as we shall show, this leads to an efficient numerical implementation. To accomplish this, we will impose a certain stability for the approximating tensors. To understand the role of stability, let us begin with some general comments about tensor approximation.

Trying to approximate a function $f$ in a Banach space $X$ in terms of (possibly short) sums of rank-one functions, which are merely required to belong to the same space $X$, is an ill-posed problem. This is well-known even in the discrete (finite-dimensional) case, see [3, 28]. For instance, the limit of a sequence of rank-two tensors may have rank three. It is also known (see [26]) that the elements of such sequences degenerate in the sense that the sums have uniformly bounded norms but the individual summands do not. This is why one resorts (predominantly in the discrete setting) to subspace-based tensor formats that are inherently more stable, although they are also more involved technically [21, 19]. Note, however, that even if one managed to stably approximate $f$ in $X$ by a short sum of arbitrary rank-one tensors in $X$, in the continuous (infinitedimensional) setting, these rank-one functions could be arbitrarily hard to compute. Thus, the rank-one functions that are allowed to enter into a competition for best tensor approximations to a given function $v$ need to be tamed in one way or another. The standard way of accomplishing this and the one we shall follow below is to add a penalty (regularization) term to the approximation error.

5.1. An alternative sparsity model. To understand the form a penalty or regularization should take, let us assume that we wish to approximate $v$ in the $\mathrm{H}^{t}$ norm. We denote by $\mathcal{T}_{r}\left(\mathrm{H}^{t}\right)$ the set 
of all rank $r$ tensors

$$
g=\sum_{k=1}^{r} g^{(k)}, \quad g^{(k)}=g_{1}^{(k)} \otimes \cdots \otimes g_{d}^{(k)}, \quad g \in \mathrm{H}^{t},
$$

and $\mathcal{T}\left(\mathrm{H}^{t}\right):=\bigcup_{r \in \mathbb{N}_{0}} \mathcal{T}_{r}\left(\mathrm{H}^{t}\right)$. When approximating $v$ by the elements from $\mathcal{T}_{r}\left(\mathrm{H}^{t}\right)$, the approximation error is not the only issue. We also want that the approximant $g$ itself to be approximable (also in $\mathrm{H}^{t}$ ). To guarantee this, we will impose regularity constraints on $g$ and therefore its components $g_{j}^{(k)}$ (see Lemma 2). To keep matters simple, we take this regularity to be membership in the space $\mathrm{H}^{t+\zeta}$ for some arbitrary but fixed $\zeta>0$. As will be seen below, in the present particular context of solving (4.1), there is a natural choice of $\zeta$. Note that one could also assume other types of regularity measures such as Besov regularity in the classical PDE setting.

We consider for any $r \in \mathbb{N}_{0}, v \in \mathrm{H}^{t}, \zeta>0$, the $K$-functional

$$
K_{r}(v, \mu):=K_{r}\left(v, \mu ; \mathrm{H}^{t}, \mathrm{H}^{t+\zeta}\right):=\inf _{g \in \mathcal{T}_{r}\left(\mathrm{H}^{t+\zeta}\right)}\left\{\|v-g\|_{t}+\mu\|g\|_{r, t+\zeta}\right\},
$$

where, for any $g \in \mathcal{T}_{r}\left(\mathrm{H}^{s}\right)$ of the form (5.1),

$$
\|g\|_{r, s}:=\inf \left[\max \left\{\|g\|_{s},\left\|g^{(k)}\right\|_{s}: k=1, \ldots, r\right\}\right],
$$

where the infimum is taken over all representations of $g$, of the form (5.1), using $r$ rank-one tensors. We infer from Lemma 3 that the infimum in $(5.2)$ is attained for some $g \in \mathcal{T}_{r}\left(\mathrm{H}^{t+\zeta}\right)$. Let us make some comments that will illuminate the role of $K_{r}$. This functional measures how well $v$ can be approximated by rank $r$ tensors (in the norm of $\mathrm{H}^{t}$ ) with the added constraint that the approximants $g$ have bounded $\mathrm{H}^{t+\zeta}$ norm. One question that arises is how should $\zeta$ be chosen. In the context of solving PDEs, to approximate the solution $u$ in a specified $\mathrm{H}^{t}$ norm with a certain rate requires some excess regularity of $u$. This excess regularity is quantified by $\zeta$. We guarantee this excess regularity by assuming some regularity for $f$ and then applying a regularity theorem, see (7.1). A second remark is that if we know that the function $f$ in (5.2) is indeed in $\mathrm{H}^{t+\zeta}$, when $\mathrm{H}^{t+\zeta}$ agrees with a classical Sobolev space, then standard constructions of approximants to $v$ would also be in $\mathrm{H}^{t+\zeta}$ and have norm in this space that does not exceed $C\|v\|_{\mathrm{H}^{t+\zeta}}$. However, we do not wish to assume that we know $\|v\|_{\mathrm{H}^{t+\zeta}}$ or $C$, which is why we resort to a general $\mu$.

Since $K_{r}(v, \mu)$ is similar to an error functional, we can obtain approximation classes in the same way we have defined the classes $\mathcal{A}^{\alpha}$ (which give error decay rate $O\left(n^{-\alpha}\right)$ ) and the more general classes $\mathcal{A}^{\gamma}$ (which give error decay rate $1 / \gamma(n)$ ). We describe this in the case of general $\gamma$ since it subsumes the special case of polynomial growth rates. We begin with any growth sequence $\{\gamma(r)\}_{r \in \mathbb{N}_{0}}, \gamma(0)=1$, that monotonically increases to infinity. Given such a $\gamma$, we define

$$
\|v\|_{\overline{\mathcal{A}}^{\gamma}\left(\mathrm{H}^{t}, \mathrm{H}^{t+\zeta}\right)}:=\sup _{r \in \mathbb{N}_{0}} \gamma(r) K_{r}\left(v, 1 / \gamma(r) ; \mathrm{H}^{t}, \mathrm{H}^{t+\zeta}\right)
$$

and the associated approximation class

$$
\overline{\mathcal{A}}^{\gamma}:=\overline{\mathcal{A}}^{\gamma}\left(\mathrm{H}^{t}, \mathrm{H}^{t+\zeta}\right):=\left\{v \in \mathrm{H}^{t}:\|v\|_{\overline{\mathcal{A}} \gamma}<\infty\right\} .
$$

We shall frequently use that, whenever $v \in \overline{\mathcal{A}}^{\gamma}\left(\mathrm{H}^{t}, \mathrm{H}^{t+\zeta}\right)$, there exists for each $r \in \mathbb{N}_{0}$ a $g_{r} \in \mathcal{T}_{r}\left(\mathrm{H}^{t}\right)$ such that

$$
\left\|v-g_{r}\right\|_{t} \leq \gamma(r)^{-1}\|v\|_{\overline{\mathcal{A}}^{\gamma}\left(\mathrm{H}^{t}, \mathrm{H}^{t+\zeta}\right)}, \quad\left\|g_{r}\right\|_{t+\zeta} \leq\left\|g_{r}\right\|_{r, t+\zeta} \leq\|v\|_{\overline{\mathcal{A}}^{\gamma}\left(\mathrm{H}^{t}, \mathrm{H}^{t+\zeta}\right)} .
$$

In other words, the approximants $g_{r}$ not only provide the approximation rate $1 / \gamma(r)$ by rank $r$ tensors but also provide a control on their regularity.

Of particular interest are sequences $(\gamma(n))_{n \geq 0}$ that have a rapid growth, because this reflects the closeness of $\mathrm{H}^{s}$-stable approximations of rank-one summands to $v$. For sequences $\gamma$ that grow faster than any polynomial rate and hence violate the requirement that

$$
\gamma(2 n) \leq C \gamma(n), \quad n \in \mathbb{N},
$$

the corresponding approximation classes are no longer linear. Nevertheless, when $\gamma(n)=\mathrm{e}^{\alpha n}$ for instance, then the sum of any two elements is still in $\overline{\mathcal{A}}^{\hat{\gamma}}$ where $\hat{\gamma}(n)=\mathrm{e}^{\frac{\alpha}{2} n}$. 
5.2. Approximations to $\mathfrak{B}^{-1}$. As in the case of Theorem 1 , the key vehicle for proving regularity theorems for the new approximation spaces are exponential sum approximations. While these have been used above for the approximation of the scalars $\lambda_{\nu}^{-1}$, we shall now employ them to construct approximate inverses to $\mathfrak{B}^{-1}$, again based on Lemma 5 . In contrast with the approximate inversion of matrices (see $[18,25]$ ), in the infinite-dimensional setting considered here, we shall take into account the mapping properties of the operator on the scale of generalized Sobolev spaces $\mathrm{H}^{t}$ with $t \in \mathbb{R}$.

In order to apply Lemma 5 for approximating the solution of $\mathfrak{B} u=f$, we recall that, by (2.24),

$$
u=\sum_{\nu \in \mathbb{N}^{d}} \lambda_{\nu}^{-1}\left\langle f, e_{\nu}\right\rangle e_{\nu}
$$

Note that by (2.16),

$$
\lambda_{\nu}^{-1} e_{\nu} \approx S_{r}\left(\lambda_{\nu}\right) e_{\nu}=\sum_{k=1}^{r} \omega_{r, k} \mathrm{e}^{-\alpha_{r, k} \lambda_{\nu}} e_{\nu}=\sum_{k=1}^{r} \omega_{r, k} \mathrm{e}^{-\alpha_{r, k} \mathfrak{B}} e_{\nu}
$$

Hence, formally, an approximation to $\mathfrak{B}^{-1} f$ is given by

$$
S_{r}(\mathfrak{B}) f:=\sum_{k=1}^{r} \omega_{r, k} \mathrm{e}^{-\alpha_{r, k} \mathfrak{B}} f .
$$

The following proposition establishes the mapping properties of the operators $S_{r}(\mathfrak{B})$ and estimates for how well this operator approximates $\mathfrak{B}^{-1}$.

Proposition 1. Let $C_{0}:=\max \left\{8, \frac{2}{\lambda}\right\}, t \in \mathbb{R}$, and $v \in \mathrm{H}^{t}$.

(i) If $t \leq s \leq t+2$, then,

$$
\left\|\mathfrak{B}^{-1}-S_{r}(\mathfrak{B})\right\|_{\mathrm{H}^{t} \rightarrow \mathrm{H}^{s}} \leq C_{0} \mathrm{e}^{-\frac{(2-(s-t)) \pi}{2} \sqrt{r}} .
$$

(ii) In particular, for any $\xi \in[0,2]$, one has

$$
\left\|\mathfrak{B}^{-1} v-S_{r}(\mathfrak{B}) v\right\|_{t+\xi} \leq C_{0} \mathrm{e}^{-\frac{(2-\xi) \pi}{2} \sqrt{r}}\|v\|_{t} .
$$

(iii) Moreover,

$$
\left\|S_{r}(\mathfrak{B}) v\right\|_{t+2} \leq\left(C_{0}+1\right)\|v\|_{t} .
$$

(iv) If $v$ is a rank-one tensor, then $S_{r}(\mathfrak{B}) v=\sum_{k=1}^{r} v^{(k)}$, where each $v^{(k)}:=\omega_{r, k} \mathrm{e}^{-\alpha_{r, k} \mathfrak{B}} v$ is a rank-one tensor, which satisfies

$$
\left\|v^{(k)}\right\|_{t+2} \leq\left(C_{0}+1\right)^{1 / 2}\|v\|_{t} .
$$

Proof. (i) Defining

we know from (4.10) that

$$
\varepsilon_{r, \nu}:=\lambda_{\nu}^{-1}-S_{r}\left(\lambda_{\nu}\right), \quad \nu \in \mathbb{N}^{d}
$$

$$
\left|\varepsilon_{r, \nu}\right| \leq \frac{16}{\underline{\lambda}} \mathrm{e}^{-\pi \sqrt{r}}, \quad \nu \in \mathbb{N}^{d}
$$

Now, we can write

$$
\mathfrak{B}^{-1} v=\sum_{\nu \in \mathbb{N}^{d}}\left\langle v, e_{\nu}\right\rangle\left(S_{r}\left(\lambda_{\nu}\right)+\varepsilon_{r, \nu}\right) e_{\nu}=S_{r}(\mathfrak{B}) v+\sum_{\nu \in \mathbb{N}^{d}} \varepsilon_{r, \nu}\left\langle v, e_{\nu}\right\rangle e_{\nu} .
$$

Hence,

$$
\begin{aligned}
\left\|\mathfrak{B}^{-1} v-S_{r}(\mathfrak{B}) v\right\|_{s} & =\left(\sum_{\nu \in \mathbb{N}^{d}} \varepsilon_{r, \nu}^{2} \lambda_{\nu}^{s}\left|\left\langle v, e_{\nu}\right\rangle\right|^{2}\right)^{1 / 2} \\
& \leq\left(\sum_{\nu \in \mathbb{N}^{d}} \lambda_{\nu}^{t}\left|\left\langle v, e_{\nu}\right\rangle\right|^{2}\right)^{1 / 2} \sup _{\nu \in \mathbb{N}^{d}}\left|\varepsilon_{r, \nu}\right| \lambda_{\nu^{\frac{s-t}{2}}} \\
& \leq\|v\|_{t} \sup _{\nu \in \mathbb{N}^{d}}\left|\varepsilon_{r, \nu}\right| \lambda_{\nu}^{\frac{s-t}{2}}
\end{aligned}
$$


We write $s-t=2-\xi$ with $\xi \in[0,2]$ and rewrite $\varepsilon_{r, \nu}^{2} \lambda_{\nu}^{s}=\varepsilon_{r, \nu}^{2} \lambda_{\nu}^{2-\xi} \lambda_{\nu}^{t}$. Now,

$$
\varepsilon_{r, \nu}^{2} \lambda_{\nu}^{s-t}=\varepsilon_{r, \nu}^{2} \lambda_{\nu}^{2-\xi}= \begin{cases}\left(\varepsilon_{r, \nu} \lambda_{\nu}\right)^{2} \lambda_{\nu}^{-\xi} \leq 8^{\xi} \mathrm{e}^{-\xi \pi \sqrt{r}}, & \lambda_{\nu}>\frac{1}{8} \mathrm{e}^{\pi \sqrt{r}}, \\ \left(\varepsilon_{r, \nu} \lambda_{\nu}\right)^{2-\xi} \varepsilon_{r, \nu}^{\xi} \leq\left[\underline{\frac{2}{\lambda}}\right]^{2-\xi} \mathrm{e}^{-\xi \pi \sqrt{r}}, & \lambda_{\nu} \leq \frac{1}{8} \mathrm{e}^{\pi \sqrt{r}},\end{cases}
$$

where in the first estimate we used the fact that $\varepsilon_{r, \nu} \lambda_{\nu} \leq 1$ because of (4.11). The second estimate used (5.14). So, (5.10) follows from (5.16). This proves (i).

(ii) The assertion (5.11) is just a restatement of (5.10).

(iii) The bound (5.12) follows from the fact that $\left\|\mathfrak{B}^{-1}\right\|_{\mathrm{H}^{t} \rightarrow \mathrm{H}^{t+2}} \leq 1$.

(iv) Each of the components $v^{(k)}$ satisfies

$$
\begin{aligned}
\left\|v^{(k)}\right\|_{t+2}^{2} & =\sum_{\nu \in \mathbb{N}^{d}} \lambda_{\nu}^{t+2}\left|\left\langle v^{(k)}, e_{\nu}\right\rangle\right|^{2} \\
& =\omega_{r, k}^{2} \sum_{\nu \in \mathbb{N}^{d}} \lambda_{\nu}^{t}\left(\lambda_{\nu} \mathrm{e}^{-\alpha_{r, k} \lambda_{\nu}}\right)^{2}\left|\left\langle v, e_{\nu}\right\rangle\right|^{2} \\
& \leq \sup _{\nu \in \mathbb{N}^{d}}\left(\omega_{r, k} \lambda_{\nu} \mathrm{e}^{-\alpha_{r, k} \lambda_{\nu}}\right)^{2}\|v\|_{t}^{2} \leq\left(1+C_{0}\right)\|v\|_{t}^{2},
\end{aligned}
$$

where we have used (2.16) in the first equality. In the last inequality, we used that, by (4.10), $x S_{r}(x) \leq 1+\frac{2}{\underline{\lambda}}$ for $x \leq \frac{1}{8} \mathrm{e}^{\pi \sqrt{r}}$ while, by (4.11), $x S_{r}(x) \leq 1$ for $x \geq \frac{1}{8} \mathrm{e}^{\pi \sqrt{r}}$ so that, for any $\nu \in \mathbb{N}^{d}$,

$$
\omega_{r, k} \lambda_{\nu} \mathrm{e}^{-\alpha_{r, k} \lambda_{\nu}} \leq \lambda_{\nu} \sum_{k=1}^{r} \omega_{r, k} \mathrm{e}^{-\alpha_{r, k} \lambda_{\nu}}=\lambda_{\nu} S_{r}\left(\lambda_{\nu}\right) \leq 1+\frac{2}{\underline{\lambda}} \leq\left(1+C_{0}\right) .
$$

Note that $\left(\mathfrak{B}^{-1}-S_{r}(\mathfrak{B})\right)_{r \in \mathbb{N}}$ as a sequence of operators from $\mathrm{H}^{t}$ to $\mathrm{H}^{s}$ tends to zero as $r \rightarrow \infty$ in the corresponding operator norm as long as $s-t<2$, while the sequence is merely uniformly bounded when $s-t=2$.

Remark 3. The statements of Proposition 1 remain valid for $s<t$ and hence $\xi>2$ where, however, the constant $C_{0}$ depends then on $\xi=2-(s-t)$, as can be seen from (5.17). Since Proposition 1 will be applied later for $s \geq t$ we are content with the above formulation to avoid further dependencies of constants.

5.3. A new regularity theorem. We can now state our new regularity theorem.

Theorem 4. Assume that $f \in \overline{\mathcal{A}}^{\gamma}\left(\mathrm{H}^{t}, \mathrm{H}^{t+\zeta}\right)$ for the specified $t \in \mathbb{R}$ and some $0<\zeta \leq 2$. Let

$$
R(r, \gamma):=\left\lceil C_{1}(\zeta)\left(\log \left(C_{0} \gamma(r)\right)\right)^{2}\right\rceil,
$$

where $C_{1}(\zeta):=\frac{4}{(\pi \zeta)^{2}}$ and $C_{0}$ is the constant in Proposition 1, and define the tempered sequence

$$
\hat{\gamma}(m):=\gamma(r), \quad r R(r, \gamma) \leq m<(r+1) R(r+1, \gamma), \quad r=1,2, \ldots
$$

Then, the solution $u$ to the variational problem (4.1) belongs to $\overline{\mathcal{A}} \hat{\gamma}\left(\mathrm{H}^{t+2}, \mathrm{H}^{t+2+\zeta}\right)$ and

$$
\|u\|_{\overline{\mathcal{A}} \bar{\gamma}\left(\mathrm{H}^{t+2}, \mathrm{H}^{t+2+\zeta}\right)} \leq\left(3+C_{0}\right)\|f\|_{\overline{\mathcal{A}}^{\gamma}\left(\mathrm{H}^{t}, \mathrm{H}^{t+\zeta}\right)},
$$

Moreover, the mapping that takes the data $f$ into a rank-r approximation to $u$, realizing the rate $\hat{\gamma}$, is continuous.

Proof. Suppose that $f \in \overline{\mathcal{A}}^{\gamma}:=\overline{\mathcal{A}}^{\gamma}\left(\mathrm{H}^{t}, \mathrm{H}^{t+\zeta}\right)$, so that, by (5.6), there exists, for each $r \in \mathbb{N}$, a $g_{r}=\sum_{k=1}^{r} g_{r}^{(k)}$, where $g_{r}^{(k)}$ is of the form (5.1), such that

$$
\left\|f-g_{r}\right\|_{t} \leq \gamma(r)^{-1}\|f\|_{\overline{\mathcal{A}}^{\gamma}}
$$

and

$$
\left\|g_{r}\right\|_{t+\zeta} \leq\left\|g_{r}\right\|_{r, t+\zeta} \leq\|f\|_{\overline{\mathcal{A}}^{\gamma}} .
$$

From the definition of $R=R(r, \gamma)$ and (5.11),

$$
\left\|\mathfrak{B}^{-1} g_{r}-S_{R}(\mathfrak{B}) g_{r}\right\|_{t+2} \leq \gamma(r)^{-1}\left\|g_{r}\right\|_{t+\zeta} .
$$


Now, we define

$$
\bar{u}:=S_{R}(\mathfrak{B}) g_{r}
$$

The bound (5.19) gives

$$
\begin{aligned}
\|u-\bar{u}\|_{t+2} & =\left\|\mathfrak{B}^{-1}(f-\mathfrak{B} \bar{u})\right\|_{t+2}=\|f-\mathfrak{B} \bar{u}\|_{t} \leq\left\|f-g_{r}\right\|_{t}+\left\|g_{r}-\mathfrak{B} S_{R}(\mathfrak{B}) g_{r}\right\|_{t} \\
& =\left\|f-g_{r}\right\|_{t}+\left\|\left(\mathfrak{B}^{-1}-S_{R}(\mathfrak{B})\right) g_{r}\right\|_{t+2} \\
& =\left\|f-g_{r}\right\|_{t}+\gamma(r)^{-1}\left\|g_{r}\right\|_{t+\zeta} \\
& \leq 2 \gamma(r)^{-1}\|f\|_{\overline{\mathcal{A}}^{\gamma}\left(\mathrm{H}^{t}, \mathrm{H}^{t+\zeta}\right)}
\end{aligned}
$$

where we have used (5.22) and (5.23) in the last step.

By construction, the rank of $\bar{u}$ is bounded by $r R$. Moreover, expanding

$$
\bar{u}=S_{R}(\mathfrak{B}) g_{r}=\sum_{k=1}^{R} \sum_{\ell=1}^{r} u_{k, \ell}, \quad \text { where } \quad u_{k, \ell}:=\omega_{R, k} \mathrm{e}^{-\alpha_{R, k} \mathfrak{B}} g_{r}^{(\ell)},
$$

we have from (iv) of Proposition 1 that

$$
\left\|u_{k, \ell}\right\|_{t+2+\zeta} \leq\left(1+C_{0}\right)^{1 / 2}\left\|g_{r}^{(\ell)}\right\|_{t+\zeta}, \quad k=1, \ldots R, \ell=1, \ldots, r .
$$

Combining this with (5.12), we obtain

$$
\|\bar{u}\|_{r R, t+2+\zeta} \leq\left(1+C_{0}\right)\left\|g_{r}\right\|_{r, t+\zeta} \leq\left(1+C_{0}\right)\|f\|_{\overline{\mathcal{A}} \gamma\left(\mathrm{H}^{t}, \mathrm{H}^{t+\zeta}\right)} .
$$

The two inequalities (5.26) and (5.29) allow us to conclude that, for any positive integer $m \in$ $[r R(r, \gamma),(r+1) R(r+1, \gamma))$,

$$
\hat{\gamma}(m) K_{m}\left(u, \hat{\gamma}(m)^{-1}, \mathrm{H}^{t+2}, \mathrm{H}^{t+2+\zeta}\right) \leq \gamma(r)\|u-\bar{u}\|_{t+2}+\|\bar{u}\|_{R r, t+2+\zeta} \leq\left(3+C_{0}\right)\|f\|_{\overline{\mathcal{A}}^{\gamma}\left(\mathrm{H}^{t}, \mathrm{H}^{t+\zeta}\right)} .
$$

Since this inequality covers all values of $m \in \mathbb{N}_{0}$, this means that

$$
u \in \overline{\mathcal{A}}^{\hat{\gamma}}\left(\mathrm{H}^{t+2}, \mathrm{H}^{t+2+\zeta}\right), \quad\|u\|_{\overline{\mathcal{A}}^{\gamma}\left(\mathrm{H}^{t+2}, \mathrm{H}^{t+2+\zeta}\right)} \leq\left(3+C_{0}\right)\|f\|_{\overline{\mathcal{A}}^{\gamma}\left(\mathrm{H}^{t}, \mathrm{H}^{t+\zeta}\right)} .
$$

The asserted continuity of the mapping that takes $g_{r}$ into $\bar{u}$ follows from the boundedness of $S_{R}(\mathfrak{B})$ as a mapping from $\mathrm{H}^{t+\zeta}$ to $\mathrm{H}^{t+2}, 0 \leq \zeta \leq 2$; c.f. Proposition 1 .

Again, Theorem 4 remains valid for $\zeta>2$ with an adjusted constant $C_{0}$, see Remark 3 . Note also that in contrast with the previous models (see Remark 2), the assumption $f \in \overline{\mathcal{A}}^{\gamma}\left(\mathrm{H}^{t}, \mathrm{H}^{t+\zeta}\right)$ does not entail increasingly stronger constraints on the approximants, and hence on $f$, when $d$ grows.

The loss in the decay rate of Theorem 4 is essentially the same as in Theorem 3. For example, any algebraic convergence rate $\gamma(r)=r^{\alpha}$ is preserved up to a logarithmic factor. Perhaps more interesting in this model is the case of very fast decay rates, as illustrated by the following example.

Example 1. If $\gamma(r)=\mathrm{e}^{\alpha r}$ for some $\alpha>0$, then one has

$$
\hat{\gamma}(r) \geq \gamma\left((r / C)^{1 / 3}\right)=\mathrm{e}^{(\alpha r / C)^{1 / 3}}
$$

where $C=C(\alpha, f, \zeta)$. Thus, on the one hand, the convergence rate for $u \in \overline{\mathcal{A}} \hat{\gamma}\left(\mathrm{H}^{t+2}, \mathrm{H}^{t+2+\zeta}\right)$ is still faster than any algebraic rate; on the other hand, in relative terms, the loss of tensor-sparsity is the larger the stronger the sparsity of the data. This is plausible since even when $f$ is a rank-one tensor, $u$ will generally have infinite rank.

The proof of (5.20) follows from the fact that $R(r, \gamma) \approx r^{2}$, with constants of equivalence depending only on $\zeta$. Hence, $r R(r, \gamma) \approx r^{3}$ and the result easily follows. 


\section{Complexity and Computational Algorithms}

While Theorem 4 says that the solution $u$ to (2.21) can be well approximated by sparse tensor sums with stable components, whenever the right-hand side has a stable representation by sparse tensor sums, it does not provide an approximation that is determined by a finite number of parameters nor does it offer a numerical algorithm for computing a finitely parametrized approximation to $u$ that meets a prescribed target accuracy. Recall, for comparison, that in low spatial dimensions, classical numerical methods and their analysis show that the smoothness or regularity of the solution to an elliptic problem determines the complexity necessary to compute an approximation to any given target accuracy.

The central question addressed in this section is whether a regularity result like Theorem 4 can be translated into an analogous statement about computation even though we are now in a highdimensional regime. We address this question in two stages. We first show in $\$ 6.2$ that whenever $f$ is in one of the approximation classes $\overline{\mathcal{A}}^{\gamma}\left(\mathrm{H}^{t}, \mathrm{H}^{t+\zeta}\right)$, for a $\gamma$ with at least power growth, then the solution $u$ can be approximated in $\mathrm{H}^{t}$ to within accuracy $\varepsilon$ by a function that is determined by $N(\varepsilon, d)$ suitable parameters where

$$
N(\varepsilon, d) \leq d^{C_{1}} \varepsilon^{-C_{2}}, \quad \varepsilon>0, d \in \mathbb{N} .
$$

In analogy with the terminology in Information-Based Complexity, we call such a result representation tractability. In this sense Theorem 4 does establish a favorable relation between regularity and complexity.

Representation complexity bounds are, unfortunately, of little practical relevance for computation, since they do not provide in general a viable and implementable numerical algorithm. In fact, the information used in deriving (6.1) is the evaluation of exponential maps $\mathrm{e}^{-\alpha \mathfrak{B}_{j}}$ applied to data approximations. Unless one knows the eigenbases of the low-dimensional component operators $\mathfrak{B}_{j}$ this is a very restrictive assumption from a practical point of view, as mentioned earlier. On the other hand, the bounds can be viewed as a benchmark for the performance of numerical schemes in more realistic scenarios. If we are only allowed to query the given data $f$ but cannot resort to an eigensystem of $\mathfrak{B}$, as is the case in any standard numerical framework for computing a solution to (2.21), then it is far less obvious how to arrive at a finitely parametrized approximation to $u$ and at what cost. We refer to the corresponding computational cost as numerical complexity. It addresses the computational complexity of approximately inverting $\mathfrak{B}$ for data with certain structural properties. This is far less studied in the context of complexity theory when $\mathfrak{B}$ cannot be diagonalized, although it is the central question in numerical computation.

We shall address numerical complexity in the remaining subsections of this section. We emphasize already here that when treating computational complexity we assume that all relevant information about the data $f$ is available to us. In particular, we do not include the question of the cost of providing approximations to $f$ in the desired format as described below. The morale of this point of view is that "data" are part of the modeling process and are given by the user. Even when the data take the simplest form, such as being a constant, conventional numerical tools would render the solution of a high-dimensional diffusion problem, with certified accuracy in a relevant norm, computationally intractable. Our main contribution, in this direction, is therefore to show that, using unconventional tools, as described in this paper, the problem is indeed numerically tractable at a computational cost not much higher than (6.1).

6.1. The right-hand side $f$. We assume that we are given an error tolerance $\varepsilon>0$ that we wish to achieve with the numerical approximation. Any numerical algorithm for solving (2.21) begins with the input of the right-hand side $f$. To exploit tensor sparsity, we need that either $f$ is itself a rank $r$ tensor for some value of $r$ or it can be well approximated by a rank $r$ tensor. Since the second case subsumes the first, we put ourselves into the second case. Namely, we know that for certain values of $\zeta$ and $\gamma$ we have $f \in \overline{\mathcal{A}}^{\gamma}\left(\mathrm{H}^{t}, \mathrm{H}^{t+\zeta}\right)$. We fix such a value $\zeta \in(0,2)$ for the excess regularity of $f$. As stressed earlier, we do not address the problem of how one would create a stable approximation to $f$, as is guaranteed by membership in this approximation class, but instead assume that such an approximation to $f$ is already given to us in this form. We will comment later on the actual feasibility of such an assumption. 
To avoid additional technicalities, we will place very mild restrictions on the sequence $\gamma$. We assume that $\gamma$ is strictly increasing and has the following two properties:

$(\gamma 1)$ : there exists a constant $\bar{C}$, depending on $\gamma$, such that

$$
\gamma^{-1}(x)+1 \leq \gamma^{-1}(\bar{C} x), \quad x \geq 1
$$

where $\gamma^{-1}$ is the inverse function of $\gamma$, i.e., $\gamma^{-1}(\gamma(x))=x$.

$(\gamma 2)$ : there exists a $\mu>0$ such that

$$
x^{\mu} / \gamma(x) \leq C, \quad x \geq 1
$$

where $C$ is a constant.

Let us note that all polynomial growth sequences are admissible and even sequences of the form $\gamma(r)=\mathrm{e}^{c r^{\beta}}, c, \beta>0$ are included. Thus, these conditions are made only to exclude very fast and slow decaying sequences. We note that the faster $\gamma$ increases the more stringent the first condition becomes. The second condition requires a minimum growth. Of course, the type of tensor approximation discussed here is of primary interest when $\gamma$ increases rapidly, so (6.3) is not a serious restriction.

In summary, in the remainder of this paper, we make the following assumption:

(A1) We assume that we are given a sequence $\gamma$, satisfying $(\gamma 1)$ and $(\gamma 2)$, a value $t \in \mathbb{R}$, and a value $\zeta \in(0,2]$. Whenever presented with an $r>0$ and $f \in \overline{\mathcal{A}}^{\gamma}\left(\mathrm{H}^{t}, \mathrm{H}^{t+\zeta}\right)$, we are given for free an approximation $g_{r}=\sum_{\ell=1}^{r} g^{(\ell)}$, satisfying:

$$
\gamma(r)\left\|f-g_{r}\right\|_{t}+\left\|g_{r}\right\|_{r, t+\zeta} \leq A_{1}\|f\|_{\overline{\mathcal{A}}^{\gamma}\left(\mathrm{H}^{t}, \mathrm{H}^{t+\zeta}\right)},
$$

with $A_{1} \geq 1$ an absolute constant.

Notice that the existence of such functions $g_{r}$ is guaranteed from the fact that $f \in \overline{\mathcal{A}}^{\gamma}\left(\mathrm{H}^{t}, \mathrm{H}^{t+\zeta}\right)$.

6.2. Representation complexity. In this subsection, we prove that for any $\varepsilon>0$, the solution to $(2.21)$ can be approximated to accuracy $\varepsilon$ by functions, which depend on a controllable number of parameters. We fix any value of $t \in \mathbb{R}$ and $0<\zeta \leq 2$ for this section. In order to render the presentation of the results of this subsection simple, we will make an assumption on the growth of the eigenvalues of $\mathfrak{B}$. This assumption will only be used in the present subsection and not in the following material, which introduces and analyzes numerical algorithms for solving (2.21). The reader can easily verify that this assumption can be generalized in many ways but always at the expense of a more complicated statement of our results.

(RA) We assume that each of the the low-dimensional factor domains $D_{j}$ is a domain in $\mathbb{R}^{p}$ with the same $p \in\{1,2,3\}$, and that the eigenvalues of $\mathfrak{B}$ satisfy, for some fixed $\beta>0$,

$$
\lambda_{m}^{*}:=\min _{j=1, \ldots, d} \lambda_{j, m} \geq \beta m^{2 / p}, \quad m \geq 1 .
$$

Classical results on eigenvalues for second order elliptic differential operators in low dimension establish (RA) for a variety of settings, see e.g. [20, 14, 15]. If the component operators were of a different order, then the form of (RA) would change but similar results could be obtained with obvious modifications.

Since the following theorem is only of a theoretical flavor, we shall take $A_{1}=1$ in the assumption (A1).

Theorem 5. Let $t \in \mathbb{R}$ and let $\zeta \in(0,2]$. Assume that (A1) holds (with constant $\left.A_{1}=1\right)$ for this $t$ and $\zeta$ and the assumption (RA) also holds. Then, for each $\varepsilon>0$, there exists a function $v(\varepsilon)$ with

$$
v(\varepsilon) \in \mathcal{T}_{\bar{r}(\varepsilon)}\left(\mathrm{H}^{t+2+\zeta}\right), \quad \bar{r}(\varepsilon) \leq 2 C_{1}(\zeta) \gamma^{-1}(4 / \varepsilon) C_{1}(\zeta)\left(\log \left(4 C_{0} / \varepsilon\right)\right)^{2},
$$

satisfying

$$
\|u-v(\varepsilon)\|_{t+2} \leq \varepsilon\|f\|_{\overline{\mathcal{A}} \gamma\left(\mathrm{H}^{t}, \mathrm{H}^{t+\zeta}\right)}
$$


and

Moreover, $v(\varepsilon)$ is determined by

$$
\|v(\varepsilon)\|_{t+2+\zeta} \leq\left(1+C_{0}\right)\|f\|_{\overline{\mathcal{A}}^{\gamma}\left(\mathrm{H}^{t}, \mathrm{H}^{t+\zeta}\right)} .
$$

$$
N(\varepsilon, d) \leq \bar{B} d \varepsilon^{-p / \zeta}\left(\gamma^{-1}(4 / \varepsilon)\right)^{1+p / \zeta}\left(\log \left(\bar{C}_{0} / \varepsilon\right)\right)^{2+2 p / \zeta}
$$

parameters, where the constant $\bar{B}$ depends on $\beta$ from (6.5), on $C_{0}$ and $\zeta$ and where $\bar{C}_{0}=4 C_{0} \bar{C}$ with $\bar{C}$ from (6.2) and $C_{0}$ from Proposition 1.

Proof. Given $\varepsilon>0$, we choose $r=r(\varepsilon)$ as the smallest integer such that $4 \leq \varepsilon \gamma(r)$, which means that

$$
r(\varepsilon):=\left\lceil\gamma^{-1}(4 / \varepsilon)\right\rceil .
$$

For this value of $r$ and this $\gamma$, we define $R$ by (5.19); in other words, $R=R(\varepsilon)$ is given by

$$
R(\varepsilon)=\left\lceil C_{1}(\zeta)\left(\log \left(4 C_{0} / \varepsilon\right)\right)^{2}\right\rceil .
$$

We take $\bar{u}=S_{R}(\mathfrak{B}) g_{r}$ where $g_{r}$ is the approximation to $f$ asserted by (A1). Arguing as in the derivation of (5.26), we find that

$$
\|u-\bar{u}\|_{t+2} \leq 2 \gamma(r)^{-1}\|f\|_{\overline{\mathcal{A}} \gamma\left(\mathrm{H}^{t}, \mathrm{H}^{t+\zeta}\right)} \leq \frac{\varepsilon}{2}\|f\|_{\overline{\mathcal{A}} \gamma\left(\mathrm{H}^{t}, \mathrm{H}^{t+\zeta}\right)} .
$$

From (5.27), we have that

$$
\bar{u}(\varepsilon)=\sum_{k=1}^{R(\varepsilon)} \sum_{\ell=1}^{r(\varepsilon)} u^{k, \ell} .
$$

Using the bounds (5.28) for the rank-one terms

$$
u^{k, \ell}=\bigotimes_{j=1}^{d} u_{j}^{k, \ell},
$$

one obtains, as in (5.29), that

$$
\|\bar{u}(\epsilon)\|_{r R, t+2+\zeta} \leq\left(1+C_{0}\right)\|f\|_{\overline{\mathcal{A}}^{\gamma}\left(\mathrm{H}^{t}, \mathrm{H}^{t+\zeta}\right)} .
$$

Now we invoke Lemma 4 , which provides for each $u_{k, \ell}$ a rank-one approximation

$$
u^{k, \ell, m}=\bigotimes_{j=1}^{d}\left(\sum_{\nu=1}^{m}\left\langle u_{j}^{k, \ell}, e_{j, \nu}\right\rangle e_{j, \nu}\right)
$$

satisfying

$$
\left\|u^{k, \ell}-u^{k, \ell, m}\right\|_{t+2} \leq\left(\lambda_{m+1}^{*}\right)^{-\zeta / 2}\left\|u^{k, \ell}\right\|_{t+2+\zeta} \leq\left(1+C_{0}\right)\left(\lambda_{m+1}^{*}\right)^{-\zeta / 2}\|f\|_{\overline{\mathcal{A}}^{\gamma}\left(\mathrm{H}^{t}, \mathrm{H}^{t+\zeta}\right)} .
$$

Hence, defining

$$
u_{m}:=\sum_{k=1}^{R(\varepsilon)} \sum_{\ell=1}^{r(\varepsilon)} u^{k, \ell, m},
$$

we have that $u_{m} \in \mathcal{T}_{r R}\left(\mathrm{H}^{t+2+\zeta}\right)$ and satisfies

$$
\left\|u-u_{m}\right\|_{t+2} \leq\left(\frac{\varepsilon}{2}+\left(1+C_{0}\right)\left(\lambda_{m+1}^{*}\right)^{-\zeta / 2} r(\varepsilon) R(\varepsilon)\right)\|f\|_{\overline{\mathcal{A}}^{\gamma}\left(\mathrm{H}^{t}, \mathrm{H}^{t+\zeta)}\right.} .
$$

We now choose $m=m(\varepsilon)$, as the smallest integer, such that

$$
\left(1+C_{0}\right)\left(\lambda_{m+1}^{*}\right)^{-\zeta / 2} r(\varepsilon) R(\varepsilon) \leq \frac{\varepsilon}{2} .
$$

If we define $v(\varepsilon):=u_{m(\varepsilon)}$, then the triangle inequality shows that (6.7) holds. The bound (6.8) follows from (6.11) since each component $u^{k, \ell, m}$, from its very definition, has smaller $\mathrm{H}^{t+2+\zeta}$ norm than that of $u^{k, \ell}$.

We now check the complexity of $v(\varepsilon)$. By (6.2), we have $r(\varepsilon)=\left\lceil\gamma^{-1}(4 / \varepsilon)\right\rceil \leq \gamma^{-1}(\bar{C} 4 / \varepsilon)$ so that

$$
\left.r(\varepsilon) R(\varepsilon)=\gamma^{-1}(4 \bar{C} / \varepsilon)\left\lceil C_{1}(\zeta)\left(\log \left(4 \bar{C} C_{0} / \varepsilon\right)\right)\right)^{2}\right\rceil .
$$


Under the assumption (6.5) the condition (6.12) is indeed satisfied provided that

$$
m(\varepsilon) \geq B \varepsilon^{-p / \zeta} \gamma^{-1}(4 / \varepsilon)^{p / \zeta}\left(\log \left(4 \bar{C} C_{0} / \varepsilon\right)\right)^{p / \zeta},
$$

where the constant $B$ depends on $\beta$ from (6.5), on $C_{0}, p$ and $\zeta$. Since the number of parameters $N(\varepsilon, d)$ needed to determine $v(\varepsilon):=u_{m(\varepsilon)}$ is at most $r(\varepsilon) R(\varepsilon) m(\varepsilon) d$ the assertion (6.9) follows from the above choices of $r(\varepsilon), R(\varepsilon)$.

The above reasoning reveals that under the assumption (A1) the representation complexity of $f$ can be bounded by $C d \varepsilon^{-p / \zeta}\left(\gamma^{-1}(4 / \varepsilon)\right)^{1+p / \zeta}$. Thus, up to a logarithmic factor, the inversion of $\mathfrak{B}$ on tensor-sparse data does not worsen the representation complexity as already hinted at by Theorem 1.

6.3. Numerical complexity. The remaining subsections of this paper are devoted to the construction of a numerical algorithm based on Theorem 4, using only queries of the approximate data $g_{r}$. This will provide, in particular, a bound on the numerical complexity of the problem (4.1). The scheme we propose in $\S 7.1$ does not require any knowledge about the eigenbases of the operators $\mathfrak{B}_{j}$ but is based on the approximate application of the operator $S_{R}(\mathfrak{B})$ to $g_{r}$.

We shall always assume the validity of (A1). In what follows let $t<0$ and let $0<\zeta \leq 2$ stand for some excess regularity. Given a target accuracy $\varepsilon>0$ we wish to formulate a numerically implementable scheme that delivers an approximation $\bar{u}(\varepsilon)$ to the solution $u$ of (4.1) of possibly low rank, satisfying

$$
\|u-\bar{u}(\varepsilon)\|_{t+2} \leq \varepsilon\|f\|_{\overline{\mathcal{A}}^{\gamma}\left(\mathrm{H}^{t}, \mathrm{H}^{t+\zeta)}\right.} .
$$

The proof of Theorem 4 will serve as the main orientation for the construction of the numerical scheme, however, with the approximate inverse $S_{R}(\mathfrak{B})$ of $\mathfrak{B}$ replaced by the variant $\bar{S}_{R}(\mathfrak{B})$ defined in Lemma 6. We will need the following counterpart to Proposition 1, which follows by combining Lemma 6 with the arguments used in the proof of Proposition 1.

Proposition 2. Fix any $\underline{a}<\pi$. For any $s>t, s-t^{\prime}<2$, there exists a constant $\bar{C}_{0}$ depending on $\underline{\lambda}, s-t^{\prime}$, and a such that

$$
\left\|\mathfrak{B}^{-1} v-\bar{S}_{r}(\mathfrak{B}) v\right\|_{s} \leq \bar{C}_{0} \mathrm{e}^{-\frac{(2-(s-t)) \underline{a}}{2} \sqrt{r}}\|v\|_{t^{\prime}}, \quad v \in \mathrm{H}^{t^{\prime}} .
$$

Moreover, $\bar{C}_{0}=\bar{C}_{0}\left(s-t^{\prime}\right)$ tends to infinity as $s-t^{\prime} \rightarrow 2$.

Now given any prescribed error tolerance $\varepsilon$, we choose

$$
r=r(\varepsilon):=\left\lceil\gamma^{-1}\left(4 A_{1} / \varepsilon\right)\right\rceil,
$$

where $A_{1}$ is the constant appearing in (A1). In other words, $r$ is the smallest integer such that

$$
\gamma(r) \geq \frac{4 A_{1}}{\varepsilon} .
$$

It follows from (A1) that $g(\varepsilon):=g_{r(\varepsilon)}$ satisfies

$$
\|f-g(\varepsilon)\|_{t} \leq \frac{\varepsilon}{4}\|f\|_{\overline{\mathcal{A}}^{\gamma}}
$$

With an eye towards (5.26) we want to choose $R=R(\varepsilon)$ such that

$$
\left\|\left(\mathfrak{B}^{-1}-\bar{S}_{R}(\mathfrak{B})\right) g_{r}\right\|_{t+2} \leq \frac{\varepsilon}{4 A_{1}}\left\|g_{r}\right\|_{t+\zeta} .
$$

By Proposition 2 and (6.17), this means that we want

$$
\bar{C}_{0} \mathrm{e}^{-\frac{a \zeta}{2} \sqrt{R}} \leq(\gamma(r))^{-1} .
$$

A suitable choice is

$$
R(\varepsilon):=\left\lceil\bar{C}_{1}(\zeta)\left(\log \left(\bar{C}_{0} \gamma(r(\varepsilon))\right)\right)^{2}\right\rceil, \quad \bar{C}_{1}(\zeta):=\frac{4}{(\underline{a} \zeta)^{2}} .
$$

Since, by (6.2), $r(\varepsilon) \leq \gamma^{-1}\left(4 \bar{C} A_{1} / \varepsilon\right)$ we conclude that

$$
\left.\left.R(\varepsilon) \leq\left\lceil\bar{C}_{1}(\zeta)\left(\log \left(\bar{C}_{2} / \varepsilon\right)\right)\right)\right)^{2}\right\rceil, \quad \bar{C}_{2}:=4 \bar{C}_{0} \bar{C} A_{1} .
$$


Then, defining the function $u(\epsilon):=\bar{S}_{R(\varepsilon)}(\mathfrak{B}) g_{r(\varepsilon)}$ and estimating as in (5.26) gives

$$
\|u-u(\varepsilon)\|_{t+2} \leq 2 A_{1} \gamma(r(\varepsilon))^{-1}\|f\|_{\overline{\mathcal{A}}^{\gamma}\left(\mathrm{H}^{t}, \mathrm{H}^{t+\zeta}\right)} \leq \frac{\varepsilon}{2}\|f\|_{\overline{\mathcal{A}}^{\gamma}} .
$$

Thus, the main issue we are faced with is how to give a numerically realizable approximation $\bar{u}(\varepsilon)$ to $u(\varepsilon)=\bar{S}_{R(\varepsilon)}(\mathfrak{B}) g_{r(\varepsilon)}$, which satisfies

$$
\|\bar{u}(\varepsilon)-u(\varepsilon)\|_{t+2} \leq \frac{\varepsilon}{2}\|f\|_{\overline{\mathcal{A}}^{\gamma}\left(\mathrm{H}^{t}, \mathrm{H}^{t+\zeta}\right)} .
$$

The following section describes a numerical algorithm for constructing such a $\bar{u}(\epsilon)$. We emphasize that although $\bar{S}_{R}(\mathfrak{B})$ is a linear operator, in order to preserve ranks, its numerical approximation will be a nonlinear process.

6.4. Numerical approximation of exponential operators. The main issue in our numerical algorithm is to provide a numerical realization of the application of the operator $\bar{S}_{R}(\mathfrak{B})$ acting on finite rank functions $g$, in our case $g=g_{r}=\sum_{\ell=1}^{r} g^{(\ell)}$. Since

$$
\bar{S}_{R}(\mathfrak{B}) g=\sum_{\ell=1}^{r} \bar{S}_{R}(\mathfrak{B}) g^{(\ell)},
$$

we need a numerical implementation of the application of the operator $\bar{S}_{R}(\mathfrak{B})$ on the rank-one tensors $g^{(\ell)}$. Given such a rank-one tensor, which we will denote by $\tau$, we have

$$
\bar{S}_{R}(\mathfrak{B}) \tau=\sum_{k=1}^{R} \bar{\omega}_{k} \mathrm{e}^{-\alpha_{k} \mathfrak{B}} \tau,
$$

where, by Lemma 6,

$$
\alpha_{k}=\alpha_{R, k}, \quad \bar{\omega}_{k}:= \begin{cases}\omega_{R, k} & \text { when } \alpha_{R, k} \geq T_{R}^{-1} \\ 0 & \text { otherwise. }\end{cases}
$$

Thus, the core task is to approximate terms of the form

$$
\mathrm{e}^{-\alpha \mathfrak{B}} \tau=\bigotimes_{j=1}^{d}\left(\mathrm{e}^{-\alpha \mathfrak{B}_{j}} \tau_{j}\right), \quad \text { where } \tau=\bigotimes_{j=1}^{d} \tau_{j}
$$

is one of the summands $g^{(\ell)}$ of $g_{r(\epsilon)}$.

In this section, we give a procedure for approximating $\mathrm{e}^{-\alpha \mathfrak{B}_{j}} \tau_{j}$ and analyze its error. This numerical procedure is based on the Dunford representation of the exponential

$$
\mathrm{e}^{-\alpha \mathfrak{B}_{j}}=\frac{1}{2 \pi i} \int_{\Gamma} \mathrm{e}^{-\alpha z}\left(z \mathfrak{I}-\mathfrak{B}_{j}\right)^{-1} \mathrm{~d} z, \quad \alpha>0,
$$

where $\mathfrak{I}$ is the identity operator (canonical injection of $H_{j}^{t+2} \rightarrow H_{j}^{t}$ ) and $\Gamma$ is a suitable curve in the right complex half-plane. Recall that the Dunford representation (6.28) holds for sectorial operators, and, in particular, for the symmetric linear elliptic operators considered here. For simplicity of exposition we shall assume that for each $j=1, \ldots, d$, we can take the same $\Gamma=\Gamma_{j}$ and that this curve is symmetric with respect to $\mathbb{R}$, see Figure 1 for an illustration. The numerical realization of the operator exponential is done in two steps. The first one employs a quadrature for the above integral with exact integrands. Since the integrands themselves are solutions of operator equations the second step consists in approximately solving these operator equations for the chosen quadrature points $z \in \Gamma$.

Quadrature methods for contour integrals of the above type are well studied, see e.g. [34, 27]. Here we follow closely the results from [23, 24, 10], which are tailored to our present needs. Specifically, as in [23], we choose for a fixed $\underline{c} \leq \underline{\lambda} / 2$, the hyperbola

$$
\Gamma(x):=\underline{c}+\cosh (x+i \pi / 6)=\underline{c}+\cosh (x) \cos (\pi / 6)+i \sinh (x) \sin (\pi / 6)
$$




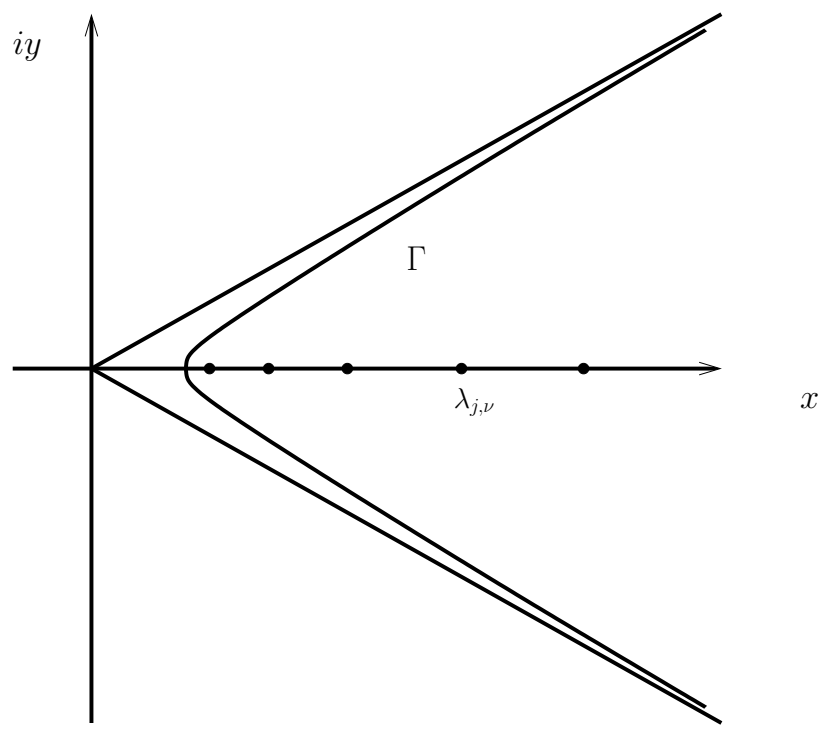

FiguRE 1. The contour $\Gamma$

as a parametrization of the curve $\Gamma$, which can be seen to have asymptotes $\underline{c}+t \mathrm{e}^{ \pm i \pi / 6}$. Denoting by $S_{b} \subset \mathbb{C}$ the symmetric strip around the real axis of width $2 b, \Gamma$ extends to a holomorphic function

$$
\Gamma(z)=\underline{c}+\cosh (x+i(\pi / 6+y)), \quad z=x+i y \in S_{b}
$$

for any $b>0$. Therefore, the operator-valued integrand (with clockwise orientation)

$$
F_{j}(z, \alpha, \cdot):=-\frac{1}{2 \pi i} \sinh (z+i \pi / 6) \mathrm{e}^{-\alpha \Gamma(z)}\left(\Gamma(z) \mathfrak{I}-\mathfrak{B}_{j}\right)^{-1}
$$

is analytic in the strip $S_{b}$ provided that $\Gamma\left(S_{b}\right)$ does not intersect the spectrum of $\mathfrak{B}_{j}$. As shown in $[23, \S 1.5 .2]$, this is ensured by choosing $b$ such that $\cos (\pi / 6-b)+\underline{c}<\underline{\lambda}$, which we will assume to hold from now on. Moreover, $\mathfrak{R e}(\Gamma(z))$ tends exponentially to infinity as $\mathfrak{R e}(z) \rightarrow \infty$.

Under these premises we know that, for $t \in \mathbb{R}$,

$$
\sup _{z \in \Gamma}\left\|\left(z \mathfrak{I}-\mathfrak{B}_{j}\right)^{-1}\right\|_{H_{j}^{t} \rightarrow H_{j}^{t+2}} \leq M, \quad j=1, \ldots, d,
$$

for some constant $M>0$.

The following result, specialized to the present setting, has been shown in [23, §1.5.4], see also [24].

Theorem 6. Let $\alpha>0, t \in \mathbb{R}$ and

Define

$$
\beta_{0}:=\frac{\alpha}{2} \cos (\pi / 6), \quad \beta_{1}:=\frac{\alpha}{2} \cos (\pi / 6+b), \quad \beta_{2}:=\frac{\alpha}{2} \cos (\pi / 6-b) .
$$

Then, for the operator

$$
C(\alpha):=\frac{M}{\pi} \mathrm{e}^{-\underline{c} \alpha}\left(\frac{1}{\beta_{0}}+\frac{\mathrm{e}^{2}}{\mathrm{e}^{2}-1}\left(\frac{1}{\beta_{1}} \mathrm{e}^{-\beta_{1}}+\frac{1}{\beta_{2}} \mathrm{e}^{-\beta_{2}}\right)\right) .
$$

one has

$$
Q_{N}\left(F_{j}(\cdot, \alpha, \cdot)\right):=h \sum_{q=-N}^{N} F_{j}(q h, \alpha, \cdot),
$$

where

$$
\left\|\mathrm{e}^{-\alpha \mathfrak{B}_{j}}-Q_{N}\left(F_{j}(\cdot, \alpha, \cdot)\right)\right\|_{H_{j}^{t} \rightarrow H_{j}^{t+2}} \leq C(\alpha) \mathrm{e}^{-2 \pi b / h}, \quad j=1, \ldots, d,
$$

$$
N=N(h):=\max \left\{0,\left\lfloor\frac{1}{h} \log \left(\beta_{0}\right)^{-1}\right\rfloor+1,\left\lfloor\frac{1}{h} \log \left(\frac{2 \pi b}{\beta_{0} h}\right)\right\rfloor+1\right\} .
$$


Notice that there exist positive constants $C_{1}, c_{1}$, depending only on $\underline{\lambda}$ and $\underline{c}$ in (6.29) such that

$$
C(\alpha) \leq \frac{C_{1}}{\alpha} \mathrm{e}^{-c_{1} \alpha}=: \bar{C}(\alpha)
$$

We also remark that one has, for $N, h$ related by (6.36),

$$
h(2 N+1) \leq C_{3}|\log (\alpha h)|,
$$

where $C_{3}$ depends only on $\underline{\lambda}$ and $\underline{c}$.

We will use the above bound also for the smaller quantities

when $0 \leq s-t^{\prime} \leq 2$.

$$
\left\|\mathrm{e}^{-\alpha \mathfrak{B}_{j}}-Q_{N}\left(F_{j}(\cdot, \alpha, \cdot)\right)\right\|_{H_{j}^{t^{\prime}} \rightarrow H_{j}^{s}},
$$

Since $Q_{N}\left(F_{j}(\cdot, \alpha, \cdot)\right)$ is an approximation to $\mathrm{e}^{-\alpha \mathfrak{B}_{j}}$, it is natural to approximate $\mathrm{e}^{-\alpha \mathfrak{B}} \tau$ by $\bigotimes_{j=1}^{d} Q_{N}\left(F_{j}(\cdot, \alpha, \cdot)\right) \tau$. The factors $Q_{N}\left(F_{j}(\cdot, \alpha, \cdot)\right)$ in the approximation to $\mathrm{e}^{-\alpha \mathfrak{B}} \tau$ are, of course, not yet computable because the quantities $u_{j, q}\left(\alpha, \tau_{j}\right):=F_{j}\left(q h, \alpha, \tau_{j}\right)$ are the exact solutions of the resolvent equations

$$
\left(\Gamma(q h) \mathfrak{I}-\mathfrak{B}_{j}\right) u_{j, q}\left(\alpha, \tau_{j}\right)=-\frac{1}{2 \pi} \sinh (q h+i \pi / 6) \mathrm{e}^{-\alpha \Gamma(q h)} \tau_{j}
$$

for $q=-N, \ldots, N$. Hence our numerical procedure will be based on approximate solutions to (6.39).

Definition 1. Let $0 \leq s, s-t^{\prime}<2$. An approximate solution $\bar{u}_{j, q}\left(\alpha, \tau_{j}\right)$ of (6.39) is called $\left(s, t^{\prime}\right)$-accurate for $\alpha$ if

$$
\left\|u_{j, q}\left(\alpha, \tau_{j}\right)-\bar{u}_{j, q}\left(\alpha, \tau_{j}\right)\right\|_{H_{j}^{s}} \leq\left(C_{3}|\log (\alpha h)|\right)^{-1} C(\alpha) \mathrm{e}^{-2 \pi b / h}\left\|\tau_{j}\right\|_{H_{j}^{t^{\prime}}},
$$

where $C_{3}$ is the constant from (6.38).

Note that here we need $s-t^{\prime}<2$ to be able to achieve a desired accuracy. We postpone at this point the discussion of how and at what cost one can obtain such approximations.

Given the approximations $\bar{u}_{j, q}\left(\alpha, \tau_{j}\right)$ of the exact integrand $u_{j, q}\left(\alpha, \tau_{j}\right)=F_{j}\left(q \pi, \alpha, \tau_{j}\right)$, we define the computable approximations

$$
E_{j}\left(\tau_{j}, \alpha, h\right):=h \sum_{q=-N}^{N} \bar{u}_{j, q}\left(\alpha, \tau_{j}\right)
$$

to $Q_{N}\left(F_{j}\left(\cdot, \alpha, \tau_{j}\right)\right)$ where $N=N(h)$, as well as

$$
E(\tau, \alpha, h):=\bigotimes_{j=1}^{d} E_{j}\left(\tau_{j}, \alpha, h\right),
$$

as a numerically realizable approximation to the rank-one tensor $\mathrm{e}^{-\alpha \mathfrak{B}} \tau$.

6.5. The numerical approximation of $u=\mathfrak{B}^{-1} f$. We are now in a position to specify our numerical approximation $u=\mathfrak{B}^{-1} f$. Recall that $u(\varepsilon)=\bar{S}_{R(\varepsilon)}(\mathfrak{B}) g_{r(\varepsilon)}$, with the choice of $R(\varepsilon)$ and $r(\varepsilon)$ specified in (6.20) and (6.16) respectively, satisfies

$$
\|u-u(\varepsilon)\|_{t+2} \leq \frac{\varepsilon}{2}\|f\|_{\overline{\mathcal{A}}^{\gamma}\left(\mathrm{H}^{t}, \mathrm{H}^{t+\zeta}\right)},
$$

because of (6.22). We will now use as our approximation to $u$ the following computable quantity

$$
\bar{S}_{R(\varepsilon), h}(\mathfrak{B})\left(g_{r(\varepsilon)}\right):=\sum_{\ell=1}^{r(\varepsilon)} \sum_{k=1}^{R(\varepsilon)} \omega_{R(\varepsilon), k} E\left(g^{(\ell)}, \alpha_{R(\varepsilon), k}, h\right) .
$$

It remains to specify $h$ sufficiently small, so as to achieve the error bound

$$
\left\|u(\varepsilon)-S_{R(\varepsilon), h}(\mathfrak{B})\left(g_{r(\varepsilon)}\right)\right\|_{t+2} \leq \frac{\varepsilon}{2}\|f\|_{\overline{\mathcal{A}}^{\gamma}\left(\mathrm{H}^{t}, \mathrm{H}^{t+\zeta}\right)} .
$$

The following result whose proof is deferred to $\S 8$ provides a sufficient choice of $h=h(\varepsilon)$. 
Proposition 3. Assume that $t+\zeta \geq 0$ and $s-(t+\zeta)<2$ and let $u(\varepsilon)$ be the approximation from (6.22). Then there exists a constant $c_{6}$, depending only on $\zeta, \bar{C}, \bar{C}_{2}$, defined in (6.21), $A_{1}$ from $(\mathbf{A} \mathbf{1})$, on $\bar{C}_{0}$ (hence depending on $\left.s-t^{\prime}>0\right), \underline{\lambda}, \Gamma$, as well as on the minimum growth of $\gamma$ in (6.3) such that for

$$
h=h(\varepsilon):=c_{6}\left(\log \left(\frac{d}{\varepsilon}\right)\right)^{-1}
$$

the function

$$
\bar{u}(\varepsilon):=S_{R(\varepsilon), h(\varepsilon)}(\mathfrak{B})\left(g_{r(\varepsilon)}\right),
$$

given by (6.44) for $h=h(\varepsilon)$, satisfies

$$
\|\bar{u}(\varepsilon)-u(\varepsilon)\|_{s} \leq \frac{\varepsilon}{2 A_{1}}\left\|g_{r(\varepsilon)}\right\|_{t+\zeta} \leq \frac{\varepsilon}{2}\|f\|_{\bar{A}^{\gamma}\left(\mathrm{H}^{t}, \mathrm{H}^{t+\zeta}\right)},
$$

provided that for $j=1, \ldots, d, \ell=1, \ldots, r(\varepsilon)$ and $q=-N(h(\varepsilon)), \ldots, N(h(\varepsilon))$, the approximate solutions $\bar{u}_{j, q}\left(\alpha_{R(\varepsilon), k}, g_{j}^{(\ell)}\right)$, entering (6.41) are $(s, t+\zeta)$ - and $(0,0)$-accurate for $\alpha_{R(\varepsilon), k}, k=$ $1, \ldots, R(\varepsilon)$.

\section{A RATE Distortion THEOREM}

Recall that the spatial dimension of the factor domains $D_{j}$ is $p \in\{1,2,3\}$ and so a key task is to approximately solve low-dimensional elliptic problems, see (6.39). The size of the discretizations of the low-dimensional operator equations (6.39), required in order to realize a desired target accuracy, depends on the regularity of the solution. In the scale of spaces $H_{j}^{t}$ and $\mathrm{H}^{t}, t \in \mathbb{R}$, this regularity follows, in view of (2.20) and analogous relations for the low-dimensional component operators, from our assumption on the right-hand side $f$ stated in assumption A1. This gives a control on the rank-one terms $g_{r}^{(\ell)}$ in the $\|\cdot\|_{t+\zeta}$ norm which, in turn, implies a regularity of $u$, see (6.8). Suitable approximations in terms of the eigensystems of the component operators $\mathfrak{B}_{j}$ could then be derived from Lemma 4. However, if one wants to dispense with using the eigensystems and, as we will do now, employ instead standard numerical techniques for low-dimensional operator equations, then one needs more information about the spaces $\mathrm{H}^{t}, H_{j}^{t}$.

Since our goal is only to illustrate that, even in the absence of having an eigensystem at hand, it is possible to construct numerical algorithms that exhibit significant computational savings when utilizing tensor structure, we shall, for the remainder of $\$ 7$, place ourselves in a more specific setting where known standard finite element solvers can be employed and bounds for their numerical efficiency are available. Specifically, we shall limit ourselves to approximating the solution in the energy norm $\mathrm{H}^{1}$. One issue to overcome is that this is not a cross norm and hence is not as compliant with tensor structures as the $\mathrm{L}_{2}$ norm. So, we are interested in approximating the solution $u$ in the $\mathrm{H}^{1}$ norm, given $\mathrm{H}^{-1+\zeta}$-data. Certain restrictions on $\zeta$ will be identified below.

Assumptions for the rate distortion theorem: We assume the validity of (A1) and that $f \in \overline{\mathcal{A}}^{\gamma}\left(\mathrm{H}^{-1}, \mathrm{H}^{-1+\zeta}\right)$. In addition, we make the following assumptions:

(A2): For $s \in[0,2]$, the spaces $H_{j}^{s}, j=1, \ldots, d$, agree with classical Sobolev spaces $H^{s}\left(D_{j}\right)$ with equivalent norms. Hence, the same holds for the spaces $\mathrm{H}^{s}, s \in[0,2]$, i.e., there exist positive constants $c^{*}, C^{*}$ such that

$$
c^{*}\|\mathfrak{B} v\|_{s-2} \leq\|v\|_{H^{s}(D)} \leq C^{*}\|\mathfrak{B} v\|_{s-2}, \quad v \in H^{s}(D), \quad 0 \leq s \leq 2 .
$$

(A3): The factor domains $D_{j}$ have the same "small" dimension $p \in\{1,2,3\}$. The operators $\mathfrak{B}_{j}$ are symmetric $H_{j}^{1}$-elliptic operators (see (2.1)) so that whenever $z \in \mathbb{C}$ satisfies $\min \left\{\left|z-\lambda_{j, k}\right|\right.$ : $k \in \mathbb{N}\}>c \mathfrak{R e}(z)$, the problem

$$
\left(z \mathfrak{I}-\mathfrak{B}_{j}\right) v=w
$$

possesses, for $j=1, \ldots, d$, and any $w \in H_{j}^{-1}$ a unique solution $v$. In particular, by (6.31), for $w \in H_{j}^{-1+\zeta}$ and $z \in \Gamma$, one has $\|v\|_{H_{j}^{1+\zeta}} \leq M\|w\|_{H_{j}^{-1+\zeta}}, j=1, \ldots, d$. 
Moreover, we have at our disposal a discretization method using linear spaces $V_{j, \delta}$ with the following properties: For any target accuracy $\delta>0$, to obtain an approximate solution $v_{\delta} \in V_{j, \delta} \subset$ $H_{j}^{1}\left(D_{j}\right)$ satisfying

$$
\left\|v-v_{\delta}\right\|_{H_{j}^{1}} \leq \delta\|w\|_{H_{j}^{-1+\zeta}}
$$

requires a trial space of dimension at most

$$
n_{\delta}:=\operatorname{dim} V_{j, \delta} \leq C_{8} \delta^{-p / \zeta}, \quad \delta>0,
$$

and the number of operations flops $\left(v_{\delta}\right)$, required to compute $v_{\delta}$, is bounded by

$$
\text { flops }\left(v_{\delta}\right) \leq C_{9} \delta^{-p / \zeta}, \quad \delta>0,
$$

with $C_{8}, C_{9}$ independent of $j=1, \ldots, d$.

Clearly, under the assumption (A2), the hypotheses in (A3) are well justified, simply resorting to available computational techniques in low spatial dimensions.

As for the justification of (A2), we state the following facts.

Remark 4. Suppose, for example, that $\mathfrak{B}_{j}$ is a second-order strongly elliptic operator of the form

$$
\mathfrak{B}_{j} v:=\sum_{k, m=1}^{p} \frac{\partial}{\partial x_{j_{k}}}\left(a_{j_{k} j_{m}}\left(x_{j_{1}}, \ldots, x_{j_{p}}\right) \frac{\partial v}{\partial x_{j_{m}}}\right), \quad j=1, \ldots, d,
$$

with $a_{j_{k} j_{m}}=a_{j_{m} j_{k}} \in C^{0,1}\left(\overline{D_{j}}\right)$ for $k, m=1, \ldots, p$, where $D_{j}$ is a bounded open convex domain in $\mathbb{R}^{p}, p \in\{1,2,3\}, j=1, \ldots, d$. Hence, $D=\times_{j=1}^{d} D_{j}$ is a bounded open convex domain (cf. [22], p. 23) and $\mathfrak{B}$ is a second-order strongly elliptic operator on D. Assuming a homogeneous Dirichlet boundary condition on $\partial D$, the second inequality in (7.1) holds with $s=s^{*}=2$ thanks to Theorem 3.2.1.2 in Grisvard [20] and the equivalence of the $H^{2}(D)$ norm on $H^{2}(D) \cap \mathrm{H}_{0}^{1}(D)$ with the standard Sobolev norm of $H^{2}(D)$. The first inequality follows trivially, by noting the regularity hypothesis on the coefficients $a_{j_{k}, j_{m}}$ and the equivalence of the $\mathrm{H}^{2}$ norm with the standard Sobolev norm of $H^{2}(D)$ on $H^{2}(D) \cap H_{0}^{1}(D)$. For $s \in(1,2), s \neq 3 / 2$, the pair of inequalities $(7.1)$ is deduced by function space interpolation. For elliptic regularity results of the kind (7.1) with $s=s^{*}=2$ for second-order degenerate elliptic operators appearing in Fokker-Planck equations we refer to $[14,15]$.

7.1. The numerical algorithm. We shall now specify the above construction of the finitely parametrized finite rank approximation $\bar{u}(\varepsilon)=S_{R(\varepsilon), h(\varepsilon)}(\mathfrak{B})\left(g_{r(\varepsilon)}\right)$ from $(6.47)$ in the following scheme.

Scheme-Exp: Given $\varepsilon>0, g(\varepsilon)=g_{r(\varepsilon)} \in \mathcal{T}_{r(\varepsilon)}\left(\mathrm{H}^{t+\zeta}\right)$ satisfying $(6.18), R=R(\varepsilon), r=r(\varepsilon)$, defined by (6.20), (6.16), the scheme produces a numerical approximation $\bar{u}(\varepsilon)$ to the solution $u$ of (4.1) as follows:

(i) Fix the curves $\Gamma_{j}=\Gamma$, according to $(6.29), j=1, \ldots, d$, along with the collections of quadrature points $\Gamma_{Q}:=\{\Gamma(q h): q=-N, \ldots, N\}, N=N(h)$ (see $\left.(6.36)\right), h=h(\varepsilon)$, given by $(6.46)$;

(ii) For $k=1, \ldots, R(\varepsilon), \ell=1, \ldots, r, q=-N, \ldots, N, j=1, \ldots, d$, compute an approximate solution $\bar{u}_{j, q}\left(\alpha_{R, k}, g_{j}^{(\ell)}\right)$ to (6.39), satisfying (6.40) for $s=1, t^{\prime}=-1+\zeta$;

(iii) compute $E_{j}\left(g_{j}^{(\ell)}, \alpha_{R, k}, h(\varepsilon)\right)$ by $(6.41)$ for $j=1, \ldots, d, \ell=1, \ldots, r, k=1, \ldots, R$, and output

$$
\bar{u}(\varepsilon):=S_{R(\varepsilon), h(\varepsilon)}(\mathfrak{B})\left(g_{r(\varepsilon)}\right)
$$

7.2. Statement of the Theorem. We can now formulate a theorem, which bounds the number of computations necessary for achieving a prescribed accuracy $\varepsilon$ using the numerical SchemeExp described above. As has been already mentioned, we describe this only for approximating the solution $u$ of (4.1) in the $\mathrm{H}^{1}$ norm. The main result of $\S 6$ reads as follows. 
Theorem 7. We assume that (A1) holds, i.e., we are given a function $f \in \overline{\mathcal{A}}^{\gamma}\left(\mathrm{H}^{-1}, \mathrm{H}^{-1+\zeta}\right)$ and for each $r \in \mathbb{N}$ a $g_{r} \in \mathcal{T}\left(\mathrm{H}^{-1+\zeta}\right)$, satisfying (6.4). Moreover, assume that $1 \leq \zeta \leq 2$ and that (A2), (A3) hold. Then, given any target accuracy $\varepsilon>0$, the approximate solution

$$
\bar{u}(\varepsilon)=S_{R(\varepsilon), h(\varepsilon)}\left(\mathfrak{B}, g_{r(\varepsilon)}\right),
$$

of rank at most $r(\varepsilon) R(\varepsilon)$, produced by Scheme-Exp has the following properties:

(i) $\bar{u}(\varepsilon)$ is $\varepsilon$-accurate and stable, i.e.,

$$
\|u-\bar{u}(\varepsilon)\|_{1} \leq \varepsilon\|f\|_{\overline{\mathcal{A}}^{\gamma}\left(\mathrm{H}^{-1}, \mathrm{H}^{-1+\zeta}\right)}, \quad\|\bar{u}(\varepsilon)\|_{r(\varepsilon) R(\varepsilon), 1} \leq A_{2}\|f\|_{\overline{\mathcal{A}}^{\gamma}\left(\mathrm{H}^{-1}, \mathrm{H}^{-1+\zeta}\right)},
$$

where $A_{2}$ depends only on $C_{0}$ and $A_{1}$.

(ii) $\bar{u}(\varepsilon)$ is finitely parametrized and the total number of parameters determining $\bar{u}(\varepsilon)$ is bounded by

$$
A_{3} d^{1+\bar{\rho} p / \zeta} \varepsilon^{-\bar{\rho} p / \zeta} \gamma^{-1}\left(4 \bar{C} A_{1} / \varepsilon\right)\left(\log \left(\frac{\bar{C}_{2}}{\varepsilon}\right)\right)^{2}\left(\log \left(\frac{d}{\varepsilon}\right)\right)^{2},
$$

where $\bar{\rho}>2 \pi b / c_{6}$ is any fixed number.

(iii) The computational complexity needed to compute $\bar{u}(\varepsilon)$ by Scheme-Exp is bounded by

$$
\operatorname{cost}(\bar{u}(\varepsilon)) \leq A_{4} d^{1+\bar{\rho} p / \zeta} \varepsilon^{-\bar{\rho} p / \zeta} \gamma^{-1}\left(4 \bar{C} A_{1} / \varepsilon\right)\left(\log \left(\frac{\bar{C}_{2}}{\varepsilon}\right)\right)^{2}\left(\log \left(\frac{d}{\varepsilon}\right)\right)^{2},
$$

where the constants $A_{3}, A_{4}$ depend on $\bar{\rho}, \underline{\lambda}, \Gamma, A_{1}$, and the constants $C_{8}, C_{9}$ in (7.3) and (7.4) in assumption (A3)).

The bounds (7.8), (7.9) given in Theorem 7 are somewhat worse than the benchmark provided by Theorem 5 and are perhaps not best possible. They rely on the specific way of approximating $\mathfrak{B}^{-1}$ and its discretization via Dunford integrals. In particular, it is not clear whether $\zeta \geq 1$ is necessary. The proof of Theorem 7, which will be given in $\S 8$, and Remark 6 below will shed some light on this constraint.

It is also not clear whether the upper limitation $\zeta \leq 2$ is necessary, i.e., whether the bounds in (7.8) and (7.9) continue to hold for $\zeta>2$, which would mean that one could exploit even higher regularity of the solutions to the resolvent equations. As will be seen below, the restriction arises when applying Proposition 3 requiring the numerical approximation to be simultaneously $(1,-1+\zeta)$ - and $(0,0)$-accurate.

Nevertheless, the theorem confirms polynomial numerical inversion tractability under overall very mild smoothness assumptions, exploiting instead a "global structural sparsity" of the solutions inferred from such a sparsity of the data. In fact, save for logarithmic terms these bounds are comparable to those for the simple example about integration given in the introduction, see (1.2). In particular, although the growth in $\varepsilon^{-1}$ and $d$ is somewhat stronger than in (1.2) or (6.9), one does benefit from a larger excess regularity at least up to $\zeta \leq 2$ of the low-dimensional problems.

Finally, in contrast with Theorem 4 the approximate solution $\bar{u}(\varepsilon)$ is only guaranteed to be tensor-stable with a controlled $\|\cdot\|_{r R, 1}$ norm not with a controlled $\|\cdot\|_{r R, 1+\zeta}$ norm. First of all, the latter bound would require the approximate solutions of the resolvent equations to belong to $H_{j}^{1+\zeta}$, which is not the case for $\zeta \geq 1 / 2$ and standard $C^{0}$-continuous finite elements. However, it can be seen from the proof of Theorem 7 that $\bar{u}(\varepsilon)$ could be arranged to satisfy $\|\bar{u}(\varepsilon)\|_{r R, 1+\zeta^{\prime}} \leq$ $C\|f\|_{\overline{\mathcal{A}}^{\gamma}\left(\mathrm{H}^{-1}, \mathrm{H}^{-1+\zeta}\right)}$ for some $0<\zeta^{\prime}<\zeta$, for which the low-dimensional trial spaces fulfill $V_{j, \delta} \subset$ $H_{j}^{1+\zeta^{\prime}}$. However, the numerical approximations to $(6.39)$ would need to be $\left(1+\zeta^{\prime},-1+\zeta\right)$-accurate and thus require possibly finer discretizations. As a consequence, the bounds (7.8) and (7.9) would only hold for $\zeta$ replaced by $\zeta-\zeta^{\prime}$. Since, the asserted tensor-stability in $\mathrm{H}^{1}$ suffices to avoid the occurrence of norm-degenerations often encountered with the canonical tensor format, we omit the details.

The results are to be contrasted with the intractability results in [30] stating that the approximation of a high-dimensional function in a Sobolev norm of positive order is intractable even under excessively high smoothness assumptions. The results in [35] on the tractability of highdimensional Helmholtz problems are different in spirit. The conditions on the data required in 
[35] strongly constrain the dependence of $f$ on some of the variables, and adopt the additional assumption that $\mathfrak{B}^{-1}$ as a mapping from such a data class into $\mathrm{H}^{1}$ can still be diagonalized. Thus the inversion of $\mathfrak{B}$, which is the focus of the present work, is not an issue there.

\section{Proof of Theorem 7}

The proof of Theorem 7 is based on a series of intermediate, partly technical results. The first of these is to give an approximation to exponential operators of the type $\mathrm{e}^{-\alpha \mathfrak{B}}$ that appear in the definition of the numerical approximation $\bar{u}(\varepsilon)$ of $(7.6)$.

8.1. Approximation of exponential operators. The present subsection and the next subsection require only the assumption (A1). A recurring issue is the need to bound the norms of $\mathrm{e}^{-\alpha \mathfrak{B}_{j}}$ and the corresponding quadrature-based counterparts as mappings from $H_{j}^{t^{\prime}}$ to $H_{j}^{s}$ also when $s>t^{\prime}$ while keeping track of the role of $\alpha>0$ when $\alpha$ gets small.

In what follows we make an effort to trace the dependence of the various constants on the problem parameters and, in particular, on $d$. To simplify the exposition somewhat we assume throughout this section that $\lambda_{j, m} \geq 1, j=1, \ldots, d, m \in \mathbb{N}$, which implies that $\underline{\lambda} \geq 1$. As a consequence we have $\|\cdot\|_{H_{j}^{s}} \leq\|\cdot\|_{H_{j}^{s^{\prime}}}$ for $s^{\prime} \geq s$. Of course, this can always be achieved by renormalizing $\mathfrak{B}$ by a fixed constant factor. Hence all subsequent conclusions remain valid in the general case up to another adjustments of the constants depending on the smallest eigenvalues.

We begin with the exact quadrature operator $Q_{N}\left(F_{j}(\cdot, \alpha, \cdot)\right)$.

Lemma 7. For any $s$ and $t^{\prime}$, satisfying $s-t^{\prime} \leq 2$, and any $\alpha>0$, one has the following bounds:

$$
\left\|\mathrm{e}^{-\alpha \mathfrak{B}_{j}}\right\|_{H_{j}^{t^{\prime}} \rightarrow H_{j}^{s}} \leq\left(\frac{\left(s-t^{\prime}\right)_{+}}{2 e \alpha}\right)^{\frac{\left(s-t^{\prime}\right)_{+}}{2}},
$$

as well as

$$
\left\|Q_{N}\left(F_{j}(\cdot, \alpha, \cdot)\right)\right\|_{H_{j}^{t^{\prime}} \rightarrow H_{j}^{s}} \leq\left(\frac{\left(s-t^{\prime}\right)_{+}}{2 e \alpha}\right)^{\frac{\left(s-t^{\prime}\right)_{+}}{2}}+\bar{C}(\alpha) \mathrm{e}^{-2 \pi b / h} .
$$

Proof. We have

$$
\left\|\mathrm{e}^{-\alpha \mathfrak{B}_{j}} \tau_{j}\right\|_{H_{j}^{s}}^{2}=\sum_{k=1}^{\infty} \mathrm{e}^{-2 \alpha \lambda_{j, k}} \lambda_{j, k}^{s-t^{\prime}} \lambda_{j, k}^{t^{\prime}}\left|\left\langle\tau_{j}, e_{j, k}\right\rangle\right|^{2} .
$$

When $s-t^{\prime} \geq 0$ we note that the function $h(x)=x^{\beta} \mathrm{e}^{-\alpha x}$ attains its maximum at $x^{*}=\beta / \alpha$, i.e.,

$$
x^{\beta} \mathrm{e}^{-\alpha x} \leq(\beta / \alpha)^{\beta} \mathrm{e}^{-\beta} .
$$

Taking $\beta=\left(s-t^{\prime}\right) / 2$ confirms (8.1) in this case. When $s-t^{\prime}<0$, we apply (8.1) for $s=t^{\prime}$ and use then that $\left\|\tau_{j}\right\|_{H_{j}^{s}} \leq\left\|\tau_{j}\right\|_{H_{j}^{t^{\prime}}}$. The bound (8.2) follows from (8.1) and Theorem 6 .

Remark 5. Note that, in particular, one has for $s \leq t^{\prime}$ the following bounds:

$$
\left\|\mathrm{e}^{-\alpha \mathfrak{B}_{j}}\right\|_{H_{j}^{t^{\prime} \rightarrow H_{j}^{s}}} \leq 1, \quad\left\|Q_{N}\left(F_{j}(\cdot, \alpha, \cdot)\right)\right\|_{H_{j}^{t^{\prime} \rightarrow H_{j}^{s}}} \leq 1+C(\alpha) \mathrm{e}^{-2 \pi b / h} .
$$

Next we shall address the effect of replacing the quantities $u_{j, q}\left(\alpha, \tau_{j}\right):=F_{j}\left(q h, \alpha, \tau_{j}\right)$, for $q=$ $-N, \ldots, N$, used in $Q_{N}\left(F_{j}\left(\cdot, \alpha, \tau_{j}\right)\right)$, which are the exact solutions of the resolvent equations (6.39), by $\left(s, t^{\prime}\right)$-accurate approximate solutions $\bar{u}_{j, q}\left(\alpha, \tau_{j}\right)$ for $\alpha$ (see $\left.(6.40)\right)$ where $s, t^{\prime}$ with $s-t^{\prime}<2$ are fixed. We shall later need this for $s=t+2$ and $t^{\prime}=t+\zeta$ with $0<\zeta \leq 2$ and will analyze the complexity of these low-dimensional problems for these choices. We recall from (6.41) the definition of the computable approximations $E_{j}\left(\tau_{j}, \alpha, h\right)$ to $Q_{N}\left(F_{j}\left(\cdot, \alpha, \tau_{j}\right)\right)$.

Lemma 8. Let $s, t^{\prime} \in \mathbb{R}$ satisfy $s-t^{\prime}<2$. Assume that (6.40) holds for $s$ and $t^{\prime}$. Then, one has

$$
\left\|\mathrm{e}^{-\alpha \mathfrak{B}_{j}} \tau_{j}-E_{j}\left(\tau_{j}, \alpha, h\right)\right\|_{H_{j}^{s}} \leq 2 \bar{C}(\alpha) \mathrm{e}^{-2 \pi b / h}\left\|\tau_{j}\right\|_{H_{j}^{t^{\prime}}},
$$

and

$$
\left\|E_{j}\left(\tau_{j}, \alpha, h\right)\right\|_{H_{j}^{s}} \leq\left\{\left(\frac{\left(s-t^{\prime}\right)_{+}}{2 \mathrm{e} \alpha}\right)^{\frac{\left(s-t^{\prime}\right)_{+}}{2}}+2 \bar{C}(\alpha) \mathrm{e}^{-2 \pi b / h}\right\}\left\|\tau_{j}\right\|_{H_{j}^{t^{\prime}}} .
$$


Proof. Using the fact that each of the terms in the sums for $E_{j}$ and $Q_{N}$ satisfies (6.40) and the number $2 N+1$ of terms satisfies (6.38), gives

$$
\left\|Q_{N}\left(F_{j}\left(\cdot, \alpha, \tau_{j}\right)\right)-E_{j}\left(\tau_{j}, \alpha, h\right)\right\|_{H_{j}^{s}} \leq C(\alpha) \mathrm{e}^{-2 \pi b / h}\left\|\tau_{j}\right\|_{H_{j}^{t^{\prime}}} .
$$

The first estimate (8.5) now follows from Theorem 6 by using the triangle inequality together with the fact that $C(\alpha) \leq \bar{C}(\alpha)$. Likewise (8.6) is a consequence of (8.5) and (8.2).

Defining $E(\tau, \alpha, h):=\bigotimes_{j=1}^{d} E_{j}\left(\tau_{j}, \alpha, h\right)$ according to (6.42), one has the following error bounds.

Lemma 9. Assume that $s \geq 0$ and $s-t^{\prime}<2$. Furthermore, assume that the approximate solutions $\bar{u}_{j, q}\left(\alpha, \tau_{j}\right)$ used in $E(\tau, \alpha, h)$ are $\left(s, t^{\prime}\right)$-accurate as well as $(0,0)$-accurate for $\alpha$ (see (6.40)). Then, whenever

holds, one has:

$$
h \leq h_{0}:=2 \pi b\left(\log \left(\frac{2 C_{1} d}{\alpha}\right)\right)^{-1}
$$

(i) for $\alpha \leq 1$,

$$
\begin{aligned}
\left\|\mathrm{e}^{-\alpha \mathfrak{B}} \tau-E(\tau, \alpha, h)\right\|_{s} \leq & C_{2} d^{2}\left(d^{\max \{0, s-1\}}\right)^{1 / 2} \bar{C}(\alpha) \mathrm{e}^{-2 \pi b / h}\left\{(\mathrm{e} \alpha)^{-1}+2 \bar{C}(\alpha) \mathrm{e}^{-2 \pi b / h}\right\} \\
& \times\left\{\left(\frac{\left(-t^{\prime}\right)_{+}}{2 \mathrm{e} \alpha}\right)^{\frac{\left(-t^{\prime}\right)_{+}}{2}}+\frac{1}{d}\right\}^{d-2}\|\tau\|_{t^{\prime}} ;
\end{aligned}
$$

(ii) for $\alpha \geq 1$,

$$
\begin{aligned}
\left\|\mathrm{e}^{-\alpha \mathfrak{B}} \tau-E(\tau, \alpha, h)\right\|_{s} \leq & C_{2} d^{2}\left(d^{\max \{0, s-1\}}\right)^{1 / 2} \bar{C}(\alpha) \mathrm{e}^{-2 \pi b / h} \\
& \times\left\{\left(\frac{\left(-t^{\prime}\right)_{+}}{2 \mathrm{e} \alpha}\right)^{\frac{\left(-t^{\prime}\right)_{+}}{2}}+\frac{1}{d}\right\}^{d-1}\|\tau\|_{t^{\prime}}
\end{aligned}
$$

where the constant $C_{2}$ depends only on the constant in Corollary 1.

Proof. We use a telescoping decomposition, as in (2.28), to obtain the bound

$$
\left\|\mathrm{e}^{-\alpha \mathfrak{B}} \tau-E(\tau, \alpha, h)\right\|_{s} \leq \sum_{i=1}^{d}\left\|S_{i}\right\|_{s}
$$

where

$$
S_{i}:=\bigotimes_{j=1}^{i-1} E_{j}\left(\tau_{j}, \alpha, h\right) \otimes\left(\mathrm{e}^{-\alpha \mathfrak{B}_{i}} \tau_{i}-E_{i}\left(\tau_{i}, \alpha, h\right)\right) \bigotimes_{j=i+1}^{d} \mathrm{e}^{-\alpha \mathfrak{B}_{j}} \tau_{j}:=\bigotimes_{j=1}^{d} S_{i, j} .
$$

We estimate next the terms $\left\|S_{i}\right\|_{s}$ with the aid of Lemma 2, using the assumption $s \geq 0$. Since the $\ell_{2}$ norm does not exceed the $\ell_{1}$ norm, this lemma gives that

$$
\left\|S_{i}\right\|_{s} \leq\left(d^{\max \{0, s-1\}}\right)^{1 / 2} \sum_{j=1}^{d}\left\|S_{i, j}\right\|_{H_{j}^{s}} \prod_{k \neq j}\left\|S_{i, k}\right\|_{L_{2}\left(D_{k}\right)} .
$$

The previous lemmas provide the following bounds for each $i=1, \ldots, d$ :

$$
\left\|S_{i, k}\right\|_{H_{k}^{s}\left(D_{k}\right)} \leq\left\|\tau_{k}\right\|_{H_{k}^{t^{\prime}\left(D_{k}\right)}} \begin{cases}2 \bar{C}(\alpha) \mathrm{e}^{-2 \pi b / h}, & k=i ; \\ \left(\frac{\left(s-t^{\prime}\right)_{+}}{2 \mathrm{e} \alpha}\right)^{\frac{\left(s-t^{\prime}\right)_{+}}{2}}+2 \bar{C}(\alpha) \mathrm{e}^{-2 \pi b / h}, & k \neq i .\end{cases}
$$

Indeed, by $\left(s, t^{\prime}\right)$-accuracy, the first inequality in (8.13) follows from (8.5) while the second follows from (8.6) and (8.1). Similarly, we have the following bounds on the $L_{2}$ norms for $\mu \in\left\{0, t^{\prime}\right\}$,

$$
\left\|S_{i, k}\right\|_{L_{2}\left(D_{k}\right)} \leq\left\|\tau_{k}\right\|_{H_{k}^{\mu}\left(D_{k}\right)} \begin{cases}2 \bar{C}(\alpha) \mathrm{e}^{-2 \pi b / h}, & k=i ; \\ \left(\frac{(-\mu)_{+}}{2 \mathrm{e} \alpha}\right)^{\frac{(-\mu)_{+}}{2}}+2 \bar{C}(\alpha) \mathrm{e}^{-2 \pi b / h}, & k \neq i .\end{cases}
$$


Here, the first inequality follows from (8.5) while the second follows from (8.6) and (8.1). In fact, since $\|\cdot\|_{L_{2}\left(D_{j}\right)} \leq\|\cdot\|_{H_{j}^{s}}$ we have used $\left(s, t^{\prime}\right)$-accuracy when $\mu=t^{\prime}$ while for $\mu=0$ we used $(0,0)$-accuracy. Moreover, under the assumption (8.7), we have

$$
2 \bar{C}(\alpha) \mathrm{e}^{-2 \pi b / h} \leq \frac{2 C_{1}}{\alpha} \mathrm{e}^{-2 \pi b / h} \leq 1 / d
$$

Case $t^{\prime} \geq 0$ : Let us first note that from the first inequality in Lemma 2, we have

$$
\sum_{i=1}^{d}\left\|\tau_{i}\right\|_{H_{i}^{t^{\prime}}} \prod_{k \neq i}\left\|\tau_{k}\right\|_{L_{2}\left(D_{k}\right)} \leq\|\tau\|_{t^{\prime}}
$$

We first consider any term appearing in the sum on the right-hand side of (8.12) with $j \neq i$ and obtain

$$
\begin{aligned}
& \left\|S_{i, j}\right\|_{H_{j}^{s}} \prod_{k \neq j}\left\|S_{i, k}\right\|_{L_{2}\left(D_{k}\right)} \\
& \leq\left\{(\mathrm{e} \alpha)^{-1}+2 \bar{C}(\alpha) \mathrm{e}^{-2 \pi b / h}\right\}\left\|\tau_{j}\right\|_{H_{j}^{t^{\prime}}}\left\{2 \bar{C}(\alpha) \mathrm{e}^{-2 \pi b / h}\left\|\tau_{i}\right\|_{L_{2}\left(D_{i}\right)}\right\} \prod_{k \neq i, j}\left(1+d^{-1}\right)\left\|\tau_{k}\right\|_{L_{2}\left(D_{k}\right)} \\
& \leq\left\{(\mathrm{e} \alpha)^{-1}+2 \bar{C}(\alpha) \mathrm{e}^{-b h}\right\}\left\{2 \bar{C}(\alpha) \mathrm{e}^{-2 \pi b / h}\right\}\left\{1+d^{-1}\right\}^{d-2}\left\|\tau_{j}\right\|_{H_{j}^{t^{\prime}}} \prod_{k \neq j}\left\|\tau_{k}\right\|_{L_{2}\left(D_{k}\right)},
\end{aligned}
$$

where we used (8.13) for the term outside of the product, and we used (8.14) with $\mu=0$ and the bound (8.15) for the remaining terms. Similarly, for the term $j=i$ in (8.12), we have

$$
\begin{aligned}
& \left\|S_{i, i}\right\|_{H_{i}^{s}} \prod_{k \neq i}\left\|S_{i, k}\right\|_{L_{2}\left(D_{k}\right)} \\
& \leq\left\{\left(2 \bar{C}(\alpha) \mathrm{e}^{-2 \pi b / h}\left\|\tau_{i}\right\|_{H_{i}^{t^{\prime}}}\right\} \prod_{k \neq i}\left(1+d^{-1}\right)\left\|\tau_{k}\right\|_{L_{2}\left(D_{k}\right)}\right. \\
& \leq\left\{2 \bar{C}(\alpha) \mathrm{e}^{-2 \pi b / h}\right\}\left\{1+d^{-1}\right\}^{d-1}\left\|\tau_{i}\right\|_{H_{i}^{t^{\prime}}} \prod_{k \neq i}\left\|\tau_{k}\right\|_{L_{2}\left(D_{k}\right)} .
\end{aligned}
$$

If $\alpha \leq 1$, we use the bounds (8.17) and (8.18) in the sum on the right-hand side of (8.12), and we arrive at

$$
\left\|S_{i}\right\|_{\mathrm{H}^{s}} \leq\left(d^{\max \{0, s-1\}}\right)^{1 / 2} d\left\{(\mathrm{e} \alpha)^{-1}+2 \tilde{C}(\alpha) \mathrm{e}^{-b h}\right\}\left\{2 \tilde{C}(\alpha) \mathrm{e}^{-b h}\right\}\left\{1+d^{-1}\right\}^{d-2}\|\tau\|_{\tau^{\prime}},
$$

where we used (8.16) and the fact that $(1+1 / d) \leq 2 e\left\{(\mathrm{e} \alpha)^{-1}+2 \tilde{C}(\alpha)\right\}$. If we now sum over $i=1, \ldots, d$, we arrive at (8.8) and complete the proof in this case. A similar argument gives the case (ii) when $\alpha>1$.

Case $t^{\prime}<0$ : The proof in this case is similar to that of Case $\mathbf{1}$ except that we use Corollary 1 in place of (8.16) and this forces us to use (8.14) for $\mu=t^{\prime}$ rather than $\mu=0$. Since we will not use this case in what follows (see Remark 6), we do not include the details.

Remark 6. In view of the value of $\bar{C}(\alpha)$ given in (6.37), the estimate (8.9) shows, in particular, that

$$
\left\|\mathrm{e}^{-\alpha \mathfrak{B}} \tau-E(\tau, \alpha, h)\right\|_{s} \leq C d^{2}\left(d^{\max \{0, s-1\}}\right)^{1 / 2} \alpha^{-1} \mathrm{e}^{-c_{1} \alpha} \mathrm{e}^{-2 \pi b / h}\|\tau\|_{t^{\prime}}, \quad \text { when } \alpha \geq 1,
$$

where $C$ depends only the embedding constant from Corollary 1 and on $C_{1}$ from (6.37). Therefore, (8.8) is of primary importance for small $\alpha$. In this regime, when $t^{\prime}<0$, the right-hand side of (8.8) contains the factor $\left(\left(\left|t^{\prime}\right| / 2 \mathrm{e} \alpha\right)^{\left|t^{\prime}\right| / 2}+(1 / d)\right)^{d-2}$. Thus, whenever $\left(\left|t^{\prime}\right| / 2 \mathrm{e} \alpha\right)>((d-1) / d)^{2 /\left|t^{\prime}\right|}$ this factor exhibits an exponential growth of the form $\alpha^{-\left|t^{\prime}\right|(d-2) / 2}$. In fact, as will be seen below, $\alpha$ can be as small as $\varepsilon^{2}$ so that, in order to compensate this growth, the factor $\mathrm{e}^{-2 \pi b / h}$ would have to satisfy at least $\mathrm{e}^{-2 \pi b / h} \lesssim \varepsilon^{\left|t^{\prime}\right|(d-2)}$. Thus, the accuracy (5.26) needed in the low-dimensional problems would scale at least like $\varepsilon^{\left|t^{\prime}\right|(d-2)}$, which is exponential in $\varepsilon$. To avoid this, at least in our proof, one apparently has to require $t^{\prime} \geq 0$. Later this means that the excess regularity $\zeta$ should satisfy $t+\zeta \geq 0$, which becomes increasingly stringent with decreasing $t$. 
Corollary 2. Under the assumptions of Lemma 9 suppose that in addition

$$
t^{\prime} \geq 0
$$

Then, for $s-t^{\prime}<2$, for which (6.40) holds, one has

$$
\left\|\mathrm{e}^{-\alpha \mathfrak{B}} \tau-E(\tau, \alpha, h)\right\|_{s} \leq C_{5} d^{2}\left(d^{\max \{0, s-1\}}\right)^{1 / 2} \max \left\{\alpha^{-1}, \alpha^{-2}\right\} \mathrm{e}^{-2 \pi b / h} \mathrm{e}^{-c_{1} \alpha}\|\tau\|_{t^{\prime}},
$$

where $C_{5}$ depends only on the embedding constant from Corollary 1 and on $\underline{\lambda}$ and $\Gamma$ through the constants in (6.37).

8.2. The analysis and proof of Proposition 3. We proceed now to the analysis of the approximation of $u$ by

$$
\bar{S}_{R(\varepsilon), h}(\mathfrak{B})\left(g_{r(\varepsilon)}\right):=\sum_{\ell=1}^{r(\varepsilon)} \sum_{k=1}^{R(\varepsilon)} \omega_{R(\varepsilon), k} E\left(g^{(\ell)}, \alpha_{R(\varepsilon), k}, h\right),
$$

as defined by (6.44). Recall that our goal is to show that when $h=h(\varepsilon)$ is given by (6.46), then we have

$$
\left\|u(\varepsilon)-S_{R(\varepsilon), h}(\mathfrak{B})\left(g_{r(\varepsilon)}\right)\right\|_{t+2} \leq \frac{\varepsilon}{2}\|f\|_{\overline{\mathcal{A}}^{\gamma}\left(\mathrm{H}^{t}, \mathrm{H}^{t+\zeta}\right)},
$$

where $u(\varepsilon)=\bar{S}_{R(\varepsilon)}(\mathfrak{B}) g_{r(\varepsilon)}$, with the choice of $R(\varepsilon)$ and $r(\varepsilon)$ specified in (6.20) and (6.16), is known to satisfy $\|u-u(\varepsilon)\|_{t+2} \leq \frac{\varepsilon}{2}\|f\|_{\overline{\mathcal{A}}^{\gamma}\left(\mathrm{H}^{t}, \mathrm{H}^{t+\zeta}\right)}$.

In going further, we recall from Lemma 6 that any summand that appears in $\bar{S}_{R(\varepsilon)}(\mathfrak{B})$ satisfies

$$
\alpha_{R(\varepsilon), k} \geq T_{R(\varepsilon)}^{-1}=8 \mathrm{e}^{-\pi \sqrt{R(\varepsilon)}} \geq 8 \mathrm{e}^{-\pi}\left(\frac{\bar{C}_{2}}{\varepsilon}\right)^{-\frac{2 \pi}{\zeta \underline{\underline{a}}}},
$$

where we have used the definition of $R(\varepsilon)$ and $C_{1}(\zeta)$ in (6.20). This means that

$$
\alpha_{R(\varepsilon), k} \geq c_{3} \varepsilon^{e(\zeta)}=: \alpha(\varepsilon), \quad e(\zeta):=\frac{2 \pi}{\zeta \underline{a}},
$$

where $c_{3}$ depends only on $\bar{C}_{0}, \bar{C}$, and $A_{1}$, see Proposition 2, (A1), and (6.2).

Proof of Proposition 3: First note that if $c_{6}$ is chosen sufficiently small, then any $h \leq h(\varepsilon)$ will comply with the threshold (8.7) required in Lemma 9. In fact, inserting the expression (8.23) into (8.7), gives

$$
h_{0}=2 \pi b\left(\log \left(\left(\frac{2 C_{1}}{c_{3}}\right)\left(\frac{d^{1 / e(\zeta)}}{\varepsilon}\right)^{e(\zeta)}\right)\right)^{-1} \geq c_{6}(\log (d / \varepsilon))^{-1}
$$

provided $c_{6}$ is chosen sufficiently small (depending in part on $\zeta$ ), because we know that $\underline{a}<\pi$. We consider only such $c_{6}$ in what follows. Therefore, Lemma 9, respectively (8.21) in Corollary 2, apply. Bearing (6.37) in mind, this yields for $r=r(\varepsilon), R=R(\varepsilon)$, given by (6.20), (6.16),

$$
\|\bar{u}(\varepsilon)-u(\varepsilon)\|_{s} \leq d^{2}\left(d^{\max \{0, s-1\}}\right)^{1 / 2} C_{5} \mathrm{e}^{-2 \pi b / h}\left(\sum_{\ell=1}^{r} \sum_{k=1}^{R} \omega_{R, k} \mathrm{e}^{-\alpha_{R, k} c_{1}} \max \left\{\alpha_{R, k}^{-1}, \alpha_{R, k}^{-2}\right\}\right)\left\|g_{r}\right\|_{r, t+\zeta} .
$$

To identify further stipulations on $c_{6}$ that eventually guarantee (6.48), we infer next from Lemma $5,(4.10)$ and (6.37) that there exists a constant $C(\Gamma)$ depending only on $\Gamma$ and $\underline{\lambda}$ such that

$$
\sum_{k=1}^{R} \omega_{R, k} \mathrm{e}^{-\alpha_{R, k} c_{1}} \leq C(\Gamma)
$$

Hence, $\|\bar{u}(\varepsilon)-u(\varepsilon)\|_{s} \leq \frac{\varepsilon}{2 A_{1}}\left\|g_{r(\varepsilon)}\right\|_{t+\zeta}$ follows provided we can show that

$$
d^{2}\left(d^{\max \{0, s-1\}}\right)^{1 / 2} C_{5} C(\Gamma) \mathrm{e}^{-2 \pi b / h} \leq \frac{\varepsilon T_{R(\varepsilon)}^{-2}}{2 A_{1} r(\varepsilon)} .
$$


Now, by the condition (6.3) on the minimum growth of $\gamma$, there exists a $\mu>0$ and a positive constant $C_{\mu}$ such that $x^{\mu} \leq C_{\mu} \gamma(x)$ for $x \geq 1$, which implies that $\gamma^{-1}(x) \leq\left(C_{\mu} x\right)^{1 / \mu}$ for $x \geq 1$. By the definition (6.16) of $r(\varepsilon)$ we thus conclude that

$$
r(\varepsilon) \leq \gamma^{-1}\left(\bar{C} 4 A_{1} / \varepsilon\right) \leq\left(\frac{4 C_{\mu} \bar{C} A_{1}}{\varepsilon}\right)^{1 / \mu} .
$$

Placing this into the right-hand side of (8.25), we are left to show that

$$
d^{2}\left(d^{\max \{0, s-1\}}\right)^{1 / 2} C_{5} C(\Gamma) \mathrm{e}^{-2 \pi b / h} \leq \frac{\varepsilon T_{R(\varepsilon)}^{-2}}{2 A_{1}}\left(\frac{4 C_{\mu} \bar{C} A_{1}}{\varepsilon}\right)^{-1 / \mu},
$$

where $\mu$ is the constant in the minimum growth condition (6.3).

Now using the bound for $T_{R(\varepsilon)}^{-1}$ given in (8.22), we need only show that

$$
d^{2}\left(d^{\max \{0, s-1\}}\right)^{1 / 2} C_{5} C(\Gamma) \mathrm{e}^{-2 \pi b / h} \leq \mathrm{e}^{-2 \pi}\left(\frac{\bar{C}_{2}}{\varepsilon}\right)^{-\frac{4 \pi}{\zeta \underline{a}}} \frac{32 \varepsilon}{A_{1}}\left(\frac{4 C_{\mu} \bar{C} A_{1}}{\varepsilon}\right)^{-1 / \mu} .
$$

Hence, it suffices to show that

$$
\mathrm{e}^{-2 \pi b / h} \leq \hat{C}\left(\frac{\varepsilon}{d}\right)^{\hat{a}}
$$

where $\hat{C}$ and $\hat{a}$ are appropriate constants. Taking a logarithm, we see that if $c_{6}$ is sufficiently small, then (8.28) will be satisfied for $h \leq c_{6} / \log \left(\frac{d}{\varepsilon}\right)$.

\subsection{Proof of Theorem 7, (i).}

Theorem 8. Assume that (A1) is valid and hence the conditions $(\gamma 1),(\gamma 2)$ hold and let $t+\zeta \geq 0$. Furthermore, assume that for $k=1, \ldots, R(\varepsilon), \ell=1, \ldots, r(\varepsilon), j=1, \ldots, d, q=-N, \ldots, N$, $N=N(h(\varepsilon))$, the approximate solutions $\bar{u}_{j, q}\left(\alpha_{R(\varepsilon), k}, g_{j}^{(\ell)}\right)$, entering the $E_{j}$ from (6.41) in the definition of $S_{R(\varepsilon), h(\varepsilon)}(\mathfrak{B})\left(g_{r(\varepsilon)}\right)$, satisfy (6.40) for the pairs

$$
s:=t+2, \quad t^{\prime}:=t+\zeta, \quad \text { and } \quad s=t^{\prime}=0 .
$$

Then, the finitely parametrized finite rank function $u(\varepsilon)$, given by (6.47), satisfies

$$
\|u-\bar{u}(\varepsilon)\|_{t+2} \leq \varepsilon\|f\|_{\overline{\mathcal{A}}^{\gamma}\left(\mathrm{H}^{t}, \mathrm{H}^{t+\zeta}\right)},
$$

and is stable in the sense that

$$
\|\bar{u}(\varepsilon)\|_{r(\varepsilon) R(\varepsilon), t+2} \leq C_{7}\|f\|_{\overline{\mathcal{A}}^{\gamma}\left(\mathrm{H}^{t}, \mathrm{H}^{t+\zeta)}\right.},
$$

where $C_{7}$ depends on $C_{0}$ and $A_{1}$.

Proof. The estimate (8.29) follows directly from (A1) and (6.48) in Proposition 3 for $s=t+2$. Concerning (8.30), we need to estimate the terms $\omega_{R(\varepsilon), k}\left\|E\left(g^{(\ell)}, \alpha_{R(\varepsilon), k}, h(\varepsilon)\right)\right\|_{t+2}$. In (5.28) we have already shown that

$$
\omega_{R(\varepsilon), k}\left\|\mathrm{e}^{-\alpha_{R(\varepsilon), k} \mathfrak{B}} g^{(\ell)}\right\|_{t+2+\zeta} \leq\left(1+C_{0}\right)\left\|g^{(\ell)}\right\|_{t+\zeta} .
$$

Hence, by Proposition 3, and in particular (8.25),

$$
\begin{aligned}
\omega_{R(\varepsilon), k} \| E & \left(g^{(\ell)}, \alpha_{R(\varepsilon), k}, h(\varepsilon)\right)\left\|_{t+2} \leq\left(1+C_{0}\right)\right\| g^{(\ell)} \|_{t+\zeta} \\
& +\omega_{R(\varepsilon), k} d^{2+(t+1) / 2} C_{5} \mathrm{e}^{-c_{1} \alpha_{R(\varepsilon), k}} \alpha_{R(\varepsilon), k}^{-2} \mathrm{e}^{-2 \pi b / h}\left\|g^{(\ell)}\right\|_{t+\zeta} \\
\leq & \left(1+C_{0}\right)\left\|g^{(\ell)}\right\|_{t+\zeta}+C(\underline{\lambda}) d^{2+(t+1) / 2} C_{5} \alpha_{R(\varepsilon), k}^{-2} \mathrm{e}^{-2 \pi b / h}\left\|g^{(\ell)}\right\|_{t+\zeta} \\
\leq & \left(1+C_{0}+\frac{\varepsilon}{2 A_{1}}\right)\left\|g^{(\ell)}\right\|_{t+\zeta},
\end{aligned}
$$

which completes the proof. 
To prove now (i) of Theorem 7 , we specialize Theorem 8 to the case $t=-1,1 \leq \zeta \leq 2$. By step (ii) of Scheme-Exp, we know that the approximate resolvent solutions are $(1,-1+\zeta)$-accurate. Thus, we only need to verify that they are automatically $(0,0)$-accurate as well. To this end, we now require also the validity of assumptions (A2), (A3). In fact, we set $\zeta:=1+\zeta^{\prime}$ for some $\zeta^{\prime} \geq 0$, and assume that the finite element solution $v_{\delta} \in V_{j, \delta}$ satisfies (7.3). By interpolation, we then obtain

$$
\left\|v-v_{\delta}\right\|_{H_{j}^{1}} \leq C \delta^{\frac{1}{\varsigma}}\|w\|_{L_{2}\left(D_{j}\right)} .
$$

Using the standard Aubin-Nitsche duality argument, this in turn, yields

$$
\left\|v-v_{\delta}\right\|_{L_{2}\left(D_{j}\right)} \leq C\left(\delta^{\frac{1}{\zeta}}\right)^{2}\|w\|_{L_{2}\left(D_{j}\right)} \leq C \delta\|w\|_{L_{2}\left(D_{j}\right)},
$$

since $\zeta \leq 2$. Hence, Theorem 8 is applicable and proves (i) of Theorem 7 .

8.4. The proof of Theorem 7, (ii) and (iii). The major part of the computational work in Scheme-Exp is obviously associated with the approximate solution of the resolvent equations (6.39), which could be done completely in parallel. Let us denote by $\operatorname{cost}(q, k, \ell, j)$ the computational cost of (6.39) for $\tau_{j}=g_{j}^{(\ell)}$ for $s=1, t^{\prime}=-1+\zeta$. Hence, the total cost of computing $\bar{u}(\varepsilon)$ is given by

$$
\operatorname{cost}(\bar{u}(\varepsilon))=\sum_{q=-N}^{N} \sum_{\ell=1}^{r(\varepsilon)} \sum_{k=1}^{R(\varepsilon)} \sum_{j=1}^{d} \operatorname{cost}(q, k, \ell, j) .
$$

In order to continue with the analysis of the computational cost (8.31) of the Scheme-Exp we require the validity of assumptions (A1) - (A3).

To estimate the cost, $\operatorname{cost}(q, k, \ell, j)$, of computing the approximate solution $\bar{u}_{j, q}\left(\alpha_{R(\varepsilon), k}, g_{j}^{(\ell)}\right)$ requires a few further preparatory remarks. First note that, by (6.29), the right-hand side of the corresponding resolvent problem (6.39) is given by

$$
\operatorname{rhs}(q, k, j, \ell):=-\frac{1}{2 \pi i} \sinh (q h(\varepsilon)+i \pi / 6) \mathrm{e}^{-\alpha_{R(\varepsilon), k} \Gamma(q h(\varepsilon))} g_{j}^{(\ell)},
$$

so that

$$
\|\operatorname{rhs}(q, k, j, \ell)\|_{H_{j}^{-1+\zeta}} \leq C_{10} \mathrm{e}^{|q h(\varepsilon)|} \mathrm{e}^{-\alpha_{R(\varepsilon), k} \mathrm{e}^{|q h(\varepsilon)| / 2}}\left\|g_{j}^{(\ell)}\right\|_{H_{j}^{-1+\zeta}},
$$

where $C_{10}$ depends only on $\Gamma$ and $\underline{\lambda}$. We record the following simple observation.

Lemma 10. For $\operatorname{rhs}(q, k, j, \ell)$ defined above and all $q=-N \ldots, N, N=N(h(\varepsilon)), j=1, \ldots, d$, $\ell=1, \ldots, R(\varepsilon), \ell=1, \ldots, r(\varepsilon)$, we have

$$
\|\operatorname{rhs}(q, k, j, \ell)\|_{H_{j}^{-1+\zeta}} \leq C_{11} \alpha_{R(\varepsilon), k}^{-1}\left\|g_{j}^{(\ell)}\right\|_{H_{j}^{-1+\zeta}},
$$

where the constant $C_{11}$ depends only on $\Gamma$ and $\underline{\lambda}$ and where $\alpha(\varepsilon)$ is defined by (8.23).

Proof. Since the function $x \mathrm{e}^{-\alpha x / 2}$ attains its maximum on $[0, \infty)$ at $x^{*}=2 / \alpha$ where it takes the value $\frac{2}{\alpha} \mathrm{e}^{-1}$ the assertion is an immediate consequence of (8.32).

Recall that by (6.40) the target accuracy depends also on $\alpha_{R(\varepsilon), k}$ but becomes more stringent as $\alpha_{R(\varepsilon), k}$ increases. Combining Lemma 10 with (6.40) shows that the target accuracy $\delta(q, k, j, \ell)$, at which the resolvent problem

$$
\left(\Gamma(g h(\varepsilon)) \mathfrak{I}-\mathfrak{B}_{j}\right) u_{j, q}\left(\alpha_{R(\varepsilon), k}, g_{j}^{(\ell)}\right)=-\frac{1}{2 \pi i} \sinh (q h(\varepsilon)+i \pi / 6) \mathrm{e}^{-\alpha_{R(\varepsilon), k} \Gamma(q h(\varepsilon))} g_{j}^{(\ell)}
$$

has to be solved in order to satisfy the tolerance required in (6.40) for $s=1, t^{\prime}=-1+\zeta$, should satisfy, in view of (6.37),

$$
C_{11}\left(\alpha_{R(\varepsilon), k}\right)^{-1} \delta(q, k, j, \ell) \leq\left(C_{3}\left|\log \left(h(\varepsilon) \alpha_{R(\varepsilon), k}\right)\right|\right)^{-1} C_{1} \alpha_{R(\varepsilon), k}^{-1} \mathrm{e}^{-2 \pi b / h(\varepsilon)} .
$$

This gives

$$
\delta(q, k, j, \ell) \leq C_{12}\left|\log \left(h(\varepsilon) \alpha_{R(\varepsilon), k}\right)\right|^{-1} \mathrm{e}^{-2 \pi b / h(\varepsilon)},
$$

where $C_{12}$ depends only on $\underline{\lambda}$ and $\Gamma$. We can now estimate the complexity of the approximate resolvent solutions. 
Lemma 11. Under the hypotheses of Theorem 7, for each $\ell=1, \ldots, r(\varepsilon), k=1, \ldots, R(\varepsilon)$, $q=-N, \ldots, N, j=1, \ldots, d$, the trial spaces $V_{q, k, \ell, j}$ required to approximate the solution to (6.39) with accuracy specified in (6.40) have dimension at most

$$
\operatorname{dim} V_{q, k, \ell, j} \leq C_{15}\left(\frac{\varepsilon}{d}\right)^{-\bar{\rho} p / \zeta} .
$$

Moreover, the computational work required to determine $\bar{u}_{j, q}\left(\alpha_{R(\varepsilon), k}, g_{j}^{(\ell)}\right)$ is bounded by

$$
\operatorname{cost}(q, k, \ell, j) \leq C_{16}\left(\frac{\varepsilon}{d}\right)^{-\bar{\rho} p / \zeta},
$$

where $\bar{\rho}$ is any fixed constant satisfying $\bar{\rho}>2 \pi b / c_{6}$, and the constants $C_{15}, C_{16}$ depend on $\bar{\rho}, C_{8}, C_{9}$, $c_{6}, c_{3}$.

Proof. We need to estimate the two factors on the right-hand side of (8.34) from below. To that end, recalling (6.46) and (6.16), we have

$$
\mathrm{e}^{-2 \pi b / h(\varepsilon)}=(\varepsilon / d)^{2 \pi b / c_{6}} .
$$

Moreover, by (6.46) and (8.23) we obtain for $\alpha_{R(\varepsilon), k} \leq 1$ (see (8.23)) that

$$
\left|\log \left(h(\varepsilon) \alpha_{R(\varepsilon), k}\right)\right| \leq\left|\log \left(\frac{c_{6} c_{3} \varepsilon^{e(\zeta)}}{\log (d / \varepsilon)}\right)\right| .
$$

Using that $\log (d / \varepsilon) \leq C(d / \varepsilon)^{\rho}$ holds for any $\rho>0$ with $C$ depending on $\rho$, we obtain

$$
\left|\log \left(h(\varepsilon) \alpha_{R(\varepsilon), k}\right)\right| \leq \log \left(\frac{C_{13} d^{\rho}}{\varepsilon^{e(\zeta)+1}}\right),
$$

where $C_{13}$ depends on $\rho, c_{3}, c_{6}$. Combining (8.37) and (8.39) it suffices to require that

$$
\delta(q, k, j, \ell) \leq\left(\frac{\varepsilon}{d}\right)^{2 \pi b / c_{6}}\left(\log \left(\frac{C_{13} d^{\rho}}{\varepsilon^{e(\zeta)+1}}\right)\right)^{-1} .
$$

Hence, choosing any fixed number $\bar{\rho}>2 \pi b / c_{6}$, there exists a constant $C_{14}$, depending on $\zeta, \rho, c_{3}, c_{6}$ such that

$$
\delta(q, k, j, \ell) \leq C_{14}\left(\frac{\varepsilon}{d}\right)^{\bar{\rho}}
$$

guarantees the validity of (6.40). The assertion of Lemma 11 follows now from (7.4) and (7.5).

We can now complete the proof of Theorem 7. In fact, in total $d r(\varepsilon) R(\varepsilon)(2 N(h(\varepsilon))+1)$ lowdimensional problems have to be solved. Recall from (6.38) that

$$
(2 N(h(\varepsilon))+1) \leq C_{3} h(\varepsilon)^{-1}|\log (h(\varepsilon) \alpha(\varepsilon))| .
$$

By (6.46) and (8.39), we conclude that

$$
(2 N(h(\varepsilon))+1) \leq C\left(\log \left(\frac{d}{\varepsilon}\right)\right)^{2},
$$

where $C$ depends on $C_{3}, c_{3}, c_{6}$, and $\rho$. Now (ii) and (iii) are immediate consequences of (8.31), Lemma 11, and the bounds (6.21), (6.16) for $R(\varepsilon), r(\varepsilon)$, respectively.

The complexity bounds are still based on generous overestimations, since the constraints on the $\delta(q, k, j, \ell)$ are uniform and refer to the least favorable constellation of parameters. Also one need not use the same number of quadrature points for all $\alpha_{R(\varepsilon), k}$. 


\section{Concluding Remarks - Some Loss of Tensor-Sparsity}

The prototypical example for the above setting is $\mathfrak{B}=-\Delta$. A natural question is what could be said about tensor-sparsity when $-\Delta$ is replaced, for example, by the second-order elliptic differential operator $w \mapsto-\operatorname{div}(A \nabla w)+w$, where $A$ is a symmetric positive definite $(d \times d)$-matrix. Intuitively, since $A$ now combines different variables one expects that the solution to

$$
-\operatorname{div}(A \nabla w)+w=g \quad \text { in } \mathbb{R}^{d}, \quad w \in H^{1}\left(\mathbb{R}^{d}\right),
$$

is "less tensor-sparse". In fact, since $A$ is symmetric positive definite, it can be factorized as $A=Q^{T} D Q$ where $Q$ is unitary. Defining $u(x):=w(Q x)=w(y),(9.1)$ takes the form

$$
-\sum_{k=1}^{d} D_{k, k} u(x)+u(x)=\mathfrak{B} u(x)=f(x):=g(Q x)
$$

where $\mathfrak{B}$ is again of the form (2.7). Hence, when $f$ in (9.2) is tensor-sparse the results above apply to (9.2) providing a tensor-sparse solution in the new coordinate system. However, this does not imply, of course, any tensor-sparsity of $w$ as a function of $y$ in the original coordinate system. Understanding the general circumstances in which it is possible to quantify any tensor-sparsity of solutions to (9.1) is the subject of ongoing work.

\section{REFERENCES}

[1] M. Bachmayr, Adaptive Low-Rank Wavelet Methods and Applications to Two-Electron Schrödinger Equations, PhD Thesis, RWTH Aachen, Oct. 2012.

[2] M. Bachmayr and W. Dahmen, Adaptive near-optimal rank tensor approximation for high-dimensional operator equations, IGPM Preprint \# 363, RWTH Aachen, April 2013. 4, 13

[3] D. Bini, M. Capovani, G. Lotti, and F. Romani, $O\left(n^{2.7799}\right)$ complexity for $n \times n$ approximate matrix multiplication. Inform. Process. Lett. 8 (1979), 234-235. 4, 13, 15

[4] D. Braess, Nonlinear Approximation Theory, Springer-Verlag, Berlin, 1986. 18

[5] D. Braess and W. Hackbusch, Approximation of $1 / x$ by exponential sums in $[1, \infty)$, IMA Journal of Numerical Analysis, 25(2005), 685-697. 14

[6] D. Braess and W. Hackbusch, On the efficient computation of high-dimensional integrals and the approximation by exponential sums. In: Multiscale, Nonlinear and Adaptive Approximation, R. DeVore and A. Kunoth, Eds. Springer Berlin Heidelberg, 2009. 14

[7] H. Brezis, Analyse fonctionnelle: Théorie et applications. Collection Mathématiques Appliquées pour la Maîtrise, Masson, Paris, 1983. 14

[8] A. Cohen, R. DeVore, C. Schwab, Analytic Regularity and Polynomial Approximation of Parametric Stochastic Elliptic PDEs, Analysis and Applications, 9(2011), 11- 47. 7

[9] A. Cohen, R. DeVore, C. Schwab, Convergence Rates of Best N-term Galerkin Approximations for a Class of Elliptic sPDEs, Foundations of Computational Mathematics, 10 (2010), 615-646. 2

[10] W. Dahmen and M. Jürgens, Error controlled regularization by projection. ETNA, 25(2006), 67-100. 2

[11] R. DeVore, Nonlinear approximation. Acta Numerica, 7(1998), 51-150. 5, 27

[12] T.J. Dijkema, Ch. Schwab, R. Stevenson, An adaptive wavelet method for solving high-dimensional elliptic PDEs, Constructive Approximation, 30, (3) (2009), 423-455. 3, 11

[13] M. Espig, Effiziente Bestapproximation mittels Summen von Elementartensoren in hohen Dimensionen. Doctoral thesis, Univ. Leipzig (2007). 3

[14] L. Figueroa and E. Süli, Greedy approximation of high-dimensional Ornstein-Uhlenbeck operators. Foundations of Computational Mathematics, 12(2012), 573-623.

[15] L. Figueroa and E. Süli, Greedy approximation of high-dimensional Ornstein-Uhlenbeck operators. arXiv: 1103.0726v1 [math.NA]. Available from: http://arxiv.org/abs/1103.0726v2 7, 13, 24, 31

[16] I.P. Gavrilyuk, W. Hackbusch and B.N. Khoromskij, Hierarchical Tensor-Product Approximation to the Inverse and Related Operators for High-Dimensional Elliptic Problems. Computing 74 (2005), 131-157. 7, 24, 31

[17] I.P. Gavrilyuk, W. Hackbusch and B.N. Khoromskij, Data-sparse approximation of a class of operator-valued functions. Math. Comp. 74 (2005), 681-708. 5, 15

[18] L. Grasedyck, Existence and Computation of a Low Kronecker-Rank Approximant to the Solution of a Tensor System with Tensor Right-Hand Side. Computing 72 (2004), 247-265. 5

[19] L. Grasedyck, Hierarchical Singular Value Decomposition of Tensors. SIAM J. Matrix Anal. Appl. 31 (2010), 2029-2054. 5, 15, 20

[20] P. Grisvard, Elliptic problems in nonsmooth domains. Monographs and Studies in Mathematics, 24. Pitman (Advanced Publishing Program), Boston, MA, 1985. 18 24, 31

[21] W. Hackbusch and S. Kühn, A New Scheme for the Tensor Representation. J. Fourier Anal. Appl.15 (2009), 706-722. 18 
[22] J.-B. Hiriart-Urruty and C. Lemaréchal, Fundamentals of convex analysis. Grundlehren Text Editions. Springer-Verlag, Berlin, 2001. 31

[23] M. Jürgens, A Semigroup Approach to the Numerical Solution of Parabolic Differential Equations. Ph.D. thesis, RWTH Aachen, 2005. 27, 28

[24] M. Jürgens, Adaptive application of the operator exponential, submitted to J. Numer. Math., special issue on Breaking Complexity: Multiscale Methods for Efficient PDE Solvers. 27, 28

[25] B.N. Khoromskij, Tensor-Structured Preconditioners and Approximate Inverse of Elliptic Operators in $\mathbb{R}^{d}$. Constr. Approx. 30 (2009), 599-620. 5, 20

[26] W.P. Krijnen, T.K. Dijkstra, and A. Stegeman, On the non-existence of optimal solutions and the occurrence of degeneracy in the Candecomp/Parafac model. Psychometrika 73 (2008), 431-439. 18

27] R. Kress, Linear Integral Equations. Springer Verlag, Berlin, 1999. 27

[28] V. De Silva and L.-H. Lim, Tensor rank and the ill-posedness of the best low-rank approximation problem, SIAM J. Matrix Anal. Appl. 30 (2008), 1084-1127. 18

[29] E. Novak and H. Woźniakowski, Tractability of Multivariate Problems, Volume I: Linear Information, EMS Tracts in Mathematics 6, EMS Publ. House, Zürich (2008). 1, 3

[30] E. Novak and H. Woźniakowski, Approximation of infinitely differentiable multivariate functions is intractable, J. Complexity 25 (2009), 398-404. 1, 32

[31] M. Reed and B. Simon, Methods of Modern Mathematical Physics. I. Second Edition. Academic Press Inc. [Harcourt Brace Jovanovich Publishers], New York, 1980. 7

[32] C. Schwab and E. Süli, Adaptive Galerkin approximation algorithms for Kolmogorov equations in infinite dimensions, Stochastic Partial Differential Equations: Analysis and Computations 1(1) (2013), 204-239. 2

[33] W. Sickel and T. Ullrich, Tensor products of Sobolev-Besov spaces and applications to approximation from the hyperbolic cross. J. Approx. Theory. 161 (2009) 748-786. 9

[34] F. Stenger, Numerical Methods based on Sinc and Analytical Functions. Springer-Verlag, New York, 1993. 27

[35] A.G. Werschulz and H. Woźniakowski, Tight tractability results for a model second-order Neumann problem, Foundations of Computational Mathematics, March 2014, DOI 10.1007/s10208-014-9195-y. 5, 32

[36] E. Zeidler, Applied Functional Analysis. Applications to Mathematical Physics. Applied Mathematical Sciences, 108, Springer-Verlag, New York, 1995. 7

Institut für Geometrie und Praktische Mathematik, RWTH Aachen, Templergraben 55, 52056 Aachen,

GERMANY, dahmen@igpm.rwth-aachen.de \& lgr@igpm.rwth-aachen.de

Department of Mathematics, Texas A \& M University, College Station, Texas 77840, USA, ronald.a.devore@gmail.com

Mathematical Institute, University of Oxford, Oxford OX2 6GG, UK, endre.suli@maths.ox.ac.uk 